


\title{
Structure, Mechanism And InhiBition \\ of the human Glutaminyl Cyclase
}

\author{
Georg-August-University Göttingen \\ Faculty of Chemistry
}

Participated in the Graduate Programme:

Metal Sites in Biomolecules

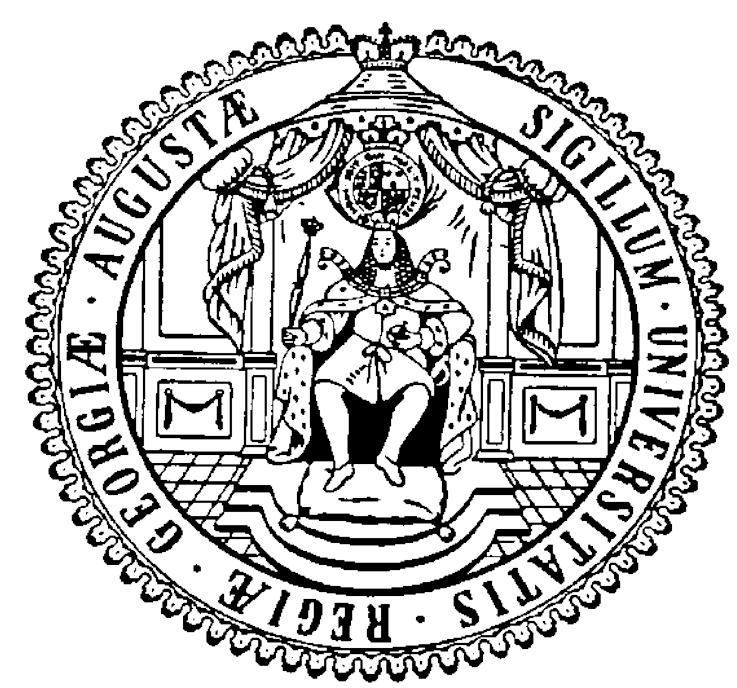

PhD Thesis

to obtain the academic degree

"Doctor rerum naturalium"

submitted by

Oliver Kupski

born on 07. July 1984

$4^{\text {th }}$ April 2016 



\section{Members of the Thesis Committee}

Prof. Dr. rer. nat. Kai Tittmann (Reviewer)

Department of Molecular Enzymology

Schwann Schleiden Research Center

Georg-August-University Göttingen

Prof. Dr. rer. nat. Franc Meyer (Reviewer)

Institute for Inorganic Chemistry

Georg-August-University Göttingen

Prof. Dr. rer. nat. Derek Logan

Department of Biochemistry and Structural Biology

Center for Molecular Protein Science

Lund University

\section{Members of the Extended Examination Board}

Prof. Dr. rer. nat. Ricardo Mata

Department of Computational Chemistry and Biochemistry Institute of Physical Chemistry Georg-August-University Göttingen 
Prof. Dr. rer. nat. Claudia Höbartner

Institute of Organic and Biomolecular Chemistry

Georg-August-University Göttingen

Dr. rer. nat. Franziska Thomas

Department of Chemical Biology and Peptide Design

Institute of Organic and Biomolecular Chemistry

Georg-August-University Göttingen

Dr. rer. nat. Fabian Commichau

Department of General Microbiology

Institute of Microbiology and Genetics

Georg-August-University Göttingen 


\section{Statutory Declaration}

Herewith I declare that I prepared this thesis "Structure, Mechanism and Inhibition of Human Glutaminyl Cyclase" independently and with no other sources and aids than quoted. This thesis (wholly or in part) has not been submitted elsewhere for any academic award or qualification.

Date: Signature: 



\section{Contents}

1 List of Figures 1

2 List of Tables 4

$\begin{array}{lll}3 & \text { Abbreviations and Symbols } & 6\end{array}$

$\begin{array}{ll}\text { Abbreviations } & 6\end{array}$

4 Introduction $\quad 10$

4.1 Pyroglutamic Acid - A Key Metabolite Within the Human System . . . . . 10

4.2 Glutaminyl Cyclase - An Enzyme Responsible For Pyroglutamic Acid Formation and Its Evolution . . . . . . . . . . . . . . . 11

4.3 Physiological Function of Mammalian Glutaminyl Cyclase . . . . . . . . . . 14

4.4 Glutaminyl Cyclase And Its Role in Neurodegenerative Diseases . . . . . . 15

4.5 Reaction Mechanism of Human Glutaminyl Cyclase . . . . . . . . . . . . . 19

4.6 Human Glutaminyl Cyclase - Target Enzyme For Drug Development . . . . 22

4.7 Motivation . . . . . . . . . . . . . . . . . . 23

5 Materials and Methods $\quad 24$

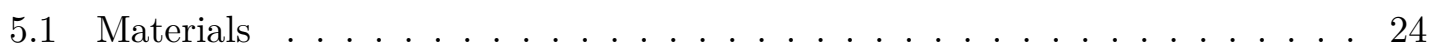

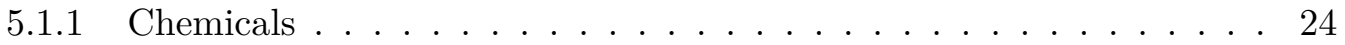

5.1 .2 Enzymes, Markers and Kit systems . . . . . . . . . . . . . . . 25

$5.1 .3 \quad$ Peptides . . . . . . . . . . . . . . . . 26

5.1 .4 Bacterial Strains . . . . . . . . . . . . . . 26

5.1 .5 Oligonucleotides . . . . . . . . . . . . . . . . 27

5.1 .6 Plasmids . . . . . . . . . . . . . . . . . 27

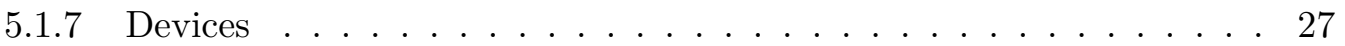

5.1 .8 Columns . . . . . . . . . . . . . . . . . . . . . 28

5.1 .9 Commodities . . . . . . . . . . . . . . . . 28

5.2 Methods . . . . . . . . . . . . . . . . . . . . . . 29

5.2 .1 Molecular Biology . . . . . . . . . . . . . . . . . . . . . . 29

5.2.1.1 Polymerase Chain Reaction (PCR) . . . . . . . . . . . 29

5.2.1.2 Cleavage of DNA with Restriction Enzymes . . . . . . . . . 29 
5.2.1.3 Agarose Gel Electrophoresis . . . . . . . . . . . . . . 30

5.2.1.4 Blunt-End Ligation of DNA Fragments . . . . . . . . . 30

5.2.1.5 Concentration and Purity Determination of DNA . . . . 30

5.2.1.6 Plasmid Transformation into Chemocompetent E.coli Cells 30

5.2.1.7 Preparation of Plasmid DNA from E.coli Cells . . . . . . . 31

5.2 .1 .8 DNA Sequencing . . . . . . . . . . . . . 31

5.2 .2 Protein Chemistry . . . . . . . . . . . . . . . . 31

5.2.2.1 Overexpression of the GST-hQC Fusion Protein in E.coli BL21 Star Cells . . . . . . . . . . . . . . . 31

5.2.2.2 Purification of the GST-hQC Fusion Protein . . . . . . . 32

5.2.2.3 Purification of Untagged hQC . . . . . . . . . . 33

5.2.2.4 Sodium Dodecyl Sulfate Polyacrylamide Gel Electrophoresis $(\mathrm{SDS}-\mathrm{PAGE}) \ldots \ldots \ldots \ldots \ldots \ldots$

5.2.2.5 Preparation of Cobalt(II)-Substituted hQC . . . . . . . 34

5.2.2.5.1 Generation of Apo-hQC . . . . . . . . . . 34

5.2.2.5.2 Reconstitution of Cobalt(II) within the Active Site 34

5.2.2.6 Determination of Protein Concentration . . . . . . . . . 34

5.2 .3 Kinetic Methods . . . . . . . . . . . . . . . . . 35

5.2.3.1 Coupled Spectroscopic Steady-State Assay . . . . . . . . 35

5.2.3.2 Continuous Calorimetric Activity Assay for Human Glutaminyl Cyclase using Isothermal Titration Calorimetry . 36

5.2.3.2.1 $\quad$ pH-Dependency of Human Glutaminyl Cyclase at Constant Ionic Strength . . . . . . . . . . . . 39

5.2.3.2.2 Kinetic Characterization of Human Glutaminyl Cyclase Inhibitors . . . . . . . . . . . . . 40

5.2.3.3 Fast Kinetics Stopped-Flow UV/Vis Spectroscopy . . . . . 40

5.2 .4 Biophysical Methods . . . . . . . . . . . . . . . . . . . 41

5.2.4.1 UV/Vis Absorption Spectroscopy . . . . . . . . . . 41

5.2.4.1.1 Titration of Cobalt to Apoenzyme . . . . . . . . 42

5.2.4.1.2 Stability of Cobalt Substituted Human Glutaminyl Cyclase . . . . . . . . . . . . . . . . . 42 
5.2.4.2 Quantitative Characterization of the Thermodynamics of Binding Events using Isothermal Titration Calorimetry . . 42

5.2.4.3 Conformational Stability of Human Glutaminyl Cyclase in Dependence of $\mathrm{pH}$ or Temperature using Circular Dichroism Spectroscopy . . . . . . . . . . . . . . . . . 43

5.2.4.4 Oligomerization State of Human Glutaminyl Cyclase Examined via Analytical Ultracentrifugation . . . . . . . . . 45

5.2.5 Liquid Chromatography - Mass Spectrometry (LC-MS) . . . . . . . 45

$5.2 .6 \quad$ X-ray Crystallography . . . . . . . . . . . . . . . . . . . 46

5.2.6.1 Crystallization and Data Collection . . . . . . . . . 46

5.2.6.2 Structure Determination and Refinement . . . . . . . . 46

6 Results and Discussion

6.1 Expression and Purification of the $\Delta 34$ Deletion Variant of Human Glutaminyl Cyclase Results in a Correctly Folded Soluble Monomer . . . . . . 48

6.2 Development of a New Steady-State Assay . . . . . . . . . . . . . . . 51

6.2.1 Motivation for Development of a Direct Continuous Enzymatic Assay for Human Glutaminyl Cyclase . . . . . . . . . . . . . . 51

6.2.2 Introduction and Overview of the Continuous Calorimetric Assay . . 52

6.2.3 First Kinetic Characterization Utlilizing Calorimetry Results in Reliable Kinetic Parameters . . . . . . . . . . . . . . . . 53

6.2.4 Verification of Applicability of the Continuous Calorimetric Assay . 55

6.3 Kinetic Characterization of Physiologic and Pathophysiologic Substrates . . 56

6.3.1 Catalytic Efficiency of N-terminal Glutaminyl Substrates in Dependence of the $\mathrm{pH} \ldots \ldots \ldots \ldots \ldots \ldots \ldots$

6.3.2 Kinetic Characterization with Different Physiological Substrates Indicates Importance of a Specific Amino Acid Pattern . . . . . . . . . 59

6.3.3 Kinetic Characterization of the Pathophysiological Substrate Shows Remarkably Decreased Catalytic Efficiency . . . . . . . . . . 61

6.3.4 Cyclization Reaction of N-Terminal Glutaminyl/Glutamyl Peptides are Spatially Separated . . . . . . . . . . . . . 63

6.3 .5 Summary ........................... 64 
6.4 The Role of the Metal Ion in Terms of Stability and Catalysis . . . . . . . . 65

6.4.1 Substitution of Zinc Against Cobalt Resulted in a Spectroscopically Active Glutaminyl Cyclase . . . . . . . . . . . . . 66

6.4.2 Incorporated Cobalt Within the Active Site of Human Glutaminyl Cyclase Shows a Moderate Tetrahedrally Coordination . . . . . . . 68

6.4.3 Coordinated Zinc Ion Plays a Role in Stabilization of the Overall Structure of Human Glutaminyl Cyclase . . . . . . . . . . . . . . 69

6.4.4 Zinc Ion Within the Active Site of hQC in Resting State Shows a Tetrahedral Coordination . . . . . . . . . . . . 70

6.4.5 Monodental Binding of N-terminal Glutaminyl Substrates Results in a Tetrahedral Coordination . . . . . . . . . . . . . . 72

6.4.6 The Cyclization Product of N-terminal Glutaminyl/Glutamyl Substrates Binds monodentally to the Catalytic Zinc Ion . . . . . . . 75

6.4.7 Coordination of the N-terminal Glutamyl Substrate Revealed a Carboxylate Shift . . . . . . . . . . . . . . 77

6.4.8 Coordination Geometry of the Metal Ion Within the Active Site Depends on Binding of Glutaminyl/Glutamyl Substrates - Role in



6.4 .9 Summary . . . . . . . . . . . . . . . . . . . . 84

6.5 Design and Characterization of a Potent Inhibitor for Human Glutaminyl



6.5.1 The First Described Imidazole Based Inhibitor for Human Glutaminyl Cyclase . . . . . . . . . . . . . . . 85

6.5.2 Screening for a New Metal Binding Group for Glutaminyl Cyclase Revealed a Carbohydrazide Function as a Potential Candidate . . . 88

6.5.3 X-Ray Structure of the Potential Carbohydrazide Inhibitor Bound by Human Glutaminyl Cyclase Revealed a Five-Fold Coordination . 93

6.5.4 Binding of the Potential Inhibitor by Human Glutaminyl Cyclase is an Entropically Driven Process . . . . . . . . . . . . 98

6.5.5 Summary - Future Aspects - Combining Backbone Group of PBD150 with the Carbohydrazide Function . . . . . . . . . . . . 99 
7 Summary and Final Discussion

8 Appendix

9 Literature

10 Acknowledgement

11 Curriculum vitae 


\section{List of Figures}

\section{List of Figures}

4.1 Chemical structure of pyroglutamic acid (5-oxo-L-proline) $\ldots \ldots \ldots$. . . . 10

4.2 Comparison of the tertiary structure of human glutaminyl cyclase and leucine aminopeptidase of Aeromonas proteolytica . . . . . . . . . . . . . . 12

4.3 Active site alignment of APAP and hQC . . . . . . . . . . . 13

4.4 Overview of catalyzed reactions by hQC $\ldots \ldots \ldots \ldots$

4.5 Schematic overview of the involvement of hQC during Alzheimer's disease development . . . . . . . . . . . . . . . . 17

4.6 Catalytic cycle of hQC catalyzing N-terminal glutaminyl substrates . . . . . 20

5.1 General overview of the spectrophotometric coupled steady-state assay . . . 35

6.1 Characterization of hQC via SDS-PAGE f . . . . . . . . . . . . 49

6.2 Far-UV spectra and thermal unfolding of hQC . . . . . . . . . . . 50

6.3 Schematic overview of the direct continuous activity assay for an exothermic reaction utilizing isothermal titration calorimetry . . . . . . . . 53

6.4 Conversion of the N-terminal glutaminyl reference substrate Gln-Gln catalyzed by hQC detected by calorimetry . . . . . . . . . . . . 54

6.5 Determination of molar reaction enthalpy after complete substrate conversion in presence of different product concentrations . . . . . . . . . 56

6.6 Catalytic efficiency of hQC in dependence of the $\mathrm{pH} \ldots \ldots \ldots$. . . . . 57

6.7 Cyclization reaction of N-terminal glutaminyl-/glutamyl substrates catalyzed by hQC . . . . . . . . . . . . . . . . . . 6 62

6.8 Scheme of protonation states for N-terminal glutaminyl/glutamyl substrates 63

6.9 Scheme of regulated exocytosis and cyclization reaction localization of $\mathrm{N}$ terminal glutaminyl/glutamyl substrates catalyzed by hQC . . . . . . . . . 64

6.10 Difference spectra of Co(II)-hQC in dependency of the cobalt concentration 66

6.11 Time-resolved absorption spectra and activity assay of $\mathrm{Co}(\mathrm{II})-\mathrm{hQC} \quad \ldots . . \quad 67$

6.12 Thermal unfolding of $\mathrm{Co}(\mathrm{II})-\mathrm{hQC}$ and apoenzyme $\ldots \ldots \ldots . . \ldots 70$

6.13 Overview of the overall crystal structure of hQC in resting state with a detailed side view of the active site . . . . . . . . . . . . . 71 
6.14 Time resolved UV/Vis absorption spectra of $\mathrm{Co}(\mathrm{II})$-hQC while catalyzing the tetrapeptide $\mathrm{QFRH} \ldots \ldots \ldots \ldots \ldots 74$

6.15 Crystal structure of hQC in complex with the tetrapeptide QFRH . . . . 75

6.16 Crystal structure of hQC in complex with the product pEFRH $\ldots \ldots$. . 76

6.17 Time resolved UV/Vis absorption spectra of $\mathrm{Co}(\mathrm{II})$-hQC after mixing with the tetrapeptide EFRH . . . . . . . . . . . . . 78

6.18 EPR spectra of Co(II)-isoDromeQC after incubation with N- terminal glutaminyl/glutamyl substrates . . . . . . . . . . . . . . . . . 79

6.19 Crystal structure of hQC in complex with the tetrapeptide EFRH . . . . 80

6.20 Crystal structure of isoDromeQC in complex with the tetrapeptide EFRH . 81

6.21 Proposed model for an induced carboxylate shift of N-terminal glutamyl substrates coordinated to the catalytic zinc ion of hQC . . . . . . . . . 84

6.22 Chemical structure of the hQC inhibitor PBD150 . . . . . . . . . 86

6.23 Crystal structure of human glutaminyl cyclase in complex with the inhibitor

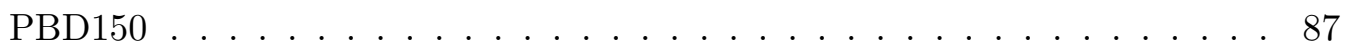

6.24 Kinetic characterization of hQC in presence of the inhibitor $\mathrm{E}$ ( $\gamma$-hydrazide)FA 92

6.25 Crystal structure of hQC in complex with the inhibitor $\mathrm{E}$ ( $\gamma$-hydrazide)FA . 93

6.26 Scheme of the Berry pseudorotation of the coordination center within the

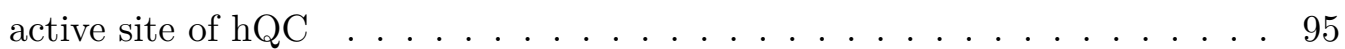

6.27 Thermodynamic binding studies of $\mathrm{E}(\gamma$-hydrazide)FA binding to hQC $\ldots 100$

7.1 Overview of the different protein folds of glutaminyl cyclase from animalia, plantae and bacteria kingdom . . . . . . . . . . . . . . . 103

8.1 Vector map of pGEX-6P-1 . . . . . . . . . . . . . . . 111

8.2 Amino acid sequence of the fusion protein GST-hQC $\mathrm{C}_{35-361} \ldots \ldots \ldots$

8.3 Amino acid sequence of $\mathrm{hQC}_{35-361} \ldots \ldots \ldots \ldots$

8.4 Kinetic characterization of hQC utilizing the spectroscopic coupled assay . 112

8.5 Controls for determination of the overall molar reaction enthalpy of hQC after substrate depletion under changing conditions . . . . . . . . . . . 113

8.6 Three-dimensional analysis of substrate depletion and product inhibition for hQC utilizing the continuous calorimetric activity assay . . . . . . . 113

8.7 hQC catalyzed cyclization reaction of N-terminal glutaminyl substrates measured via the continuous calorimetric activity assay $\ldots \ldots$. . . . . 114 
8.8 Difference spectra of Co(II)-hQC in dependency of the cobalt concentration 115

8.9 Kinetic studies of cobalt association and dissociation . . . . . . . . . 115

8.10 Active site alignment of hQC and isoDromeQC . . . . . . . . . . . . 116

8.11 CD-spectra of hQC, Co(II)-hQC and apoenzyme before and after thermal



8.12 Thermodynamic characterization of binding of $\mathrm{E}(\gamma$-hydrazide $) \mathrm{FA}$ to hQC via isothermal titration calorimetry . . . . . . . . . . . . . 117

8.13 Three-dimensional analysis of $\mathrm{E}(\gamma$-hydrazide $) \mathrm{FA}$ binding to hQC $\ldots . . .117$

8.14 Kinetic characterization of hQC in presence of different inhibitors using the calorimetric activity assay . . . . . . . . . . . . . . . . . . 119 


\section{List of Tables}

\section{List of Tables}

5.1 Temperature protocol for PCR reaction . . . . . . . . . . . . . . 29

5.2 ITC setup for single injection experiments . . . . . . . . . . . . . 39

5.3 Used concentrations for the continuous calorimetric activity assay . . . . . . 39

5.4 Final concentration of compounds after mixing in the measurement cell . . 41

5.5 Used ITC setup and protein-ligand concentrations for thermodynamic char-



5.6 Settings of the CD-spectrometer for the thermal denaturation. . . . . . . . 45

5.7 Substrate soaking conditions and data collection information for all presented crystal structures . . . . . . . . . . . . . . . . 47

6.1 Determined kinetic parameters for hQC catalyzing N-terminal glutaminyl substrates in dependence of the $\mathrm{pH} \ldots \ldots \ldots$. . . . . . . . . 58

6.2 Determined kinetic parameters for hQC catalyzing N-terminal glutaminyl/glutamyl

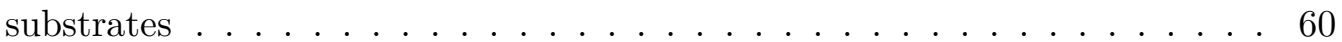

6.3 Overview of tested inhibitors for hQC $\ldots \ldots \ldots \ldots$

7.1 Overview of kinetic parameters of glutaminyl cyclase from different domains of life catalyzing N-terminal glutaminyl substrates . . . . . . . . . . . 102

8.1 Determined bond length of the metal complex in hQC after coordination of different ligands . . . . . . . . . . . . . . . . . . . . . . . 118

8.2 Determined angles of the metal complex in hQC after coordination of different ligands . . . . . . . . . . . . . . . . . . . . 118

8.3 Crystallographic data and refinement statistics for hQC in resting state and in complex with different ligands . . . . . . . . . . . . . . . . . . . 120 



\section{Abbreviations and Symbols}

\section{Abbreviations}

$A \beta$

$A_{\text {spec }}$

AD

APAP

APS

APP

ATP

CD

$\mathrm{Cl}$

Co

$\mathrm{CoCl}_{2}$

Coot

DNA

DNaseI

DTT

dp

EDTA

EPR

GnRH

GLDH

Gln

Glu

pGlu

GST

$\mathrm{HCl}$

hQC

IPTG

ITC

$k_{\text {cat }}$
Amyloid-beta peptide

Specific activity in $\mathrm{U} / \mathrm{mg}$

Alzheimer's disease

Aminopeptidase from Aeromonas proteolytica

Ammonium peroxodisulfate

Amyloid precursor protein

Adenosine triphosphate

Circular dichroism

Chloride

Cobalt

Cobalt chloride

Crystallographic object-oriented toolkit

Deoxyribonucleic acid

Deoxyribonuclease I

Dithiothreitol

Differential power in $\mu \mathrm{cal} / \mathrm{s}$

Ethylenediaminetetraacetic acid

Electron paramagnetic resonance

Gonadotropin-releasing hormone

Glutamate dehydrogenase

Glutamine

Glutamate

Pyroglutamic acid

Glutathione S-transferase

Hydrochloric acid

Human glutaminyl cyclase

Isopropyl ß-D-1-thiogalactopyranoside

Isothermal titration calorimetry

Turnover number in $\mathrm{s}^{-1}$ 


$\begin{array}{ll}k_{\mathbf{c a t}} / K_{\mathbf{M}} & \text { Catalytic efficiency in } \mathrm{s}^{-1} \mathrm{mM}^{-1} \\ K_{\mathbf{d}} & \text { Dissociation constant } \\ K_{\mathbf{i}} & \text { Inhibition constant } \\ K_{\mathbf{M}} & \text { Michaelis-Menten constant } \\ \mathbf{L B} & \text { Lysogeny broth } \\ \mathbf{M E S} & 2 \text {-(N-morpholino)ethanesulfonic acid }\end{array}$

$\mathrm{MgSO}_{4}$

Magnesium sulfate

mRNA

Messenger ribonucleic acid

MW

Molecular weight

MWCO

Molecular weight cut-off

$\mathrm{NaCl}$

Sodium chloride

NADH

Nicotinamide adenine dinucleotide (reduced form)

NAD $^{+}$

Nicotinamide adenine dinucleotide (oxidized form)

BNA

Beta-naphthalene

NTP

Nucleoside-5'-triphosphate

$\mathrm{NaH}_{2} \mathrm{PO}_{4}$

Sodium dihydrogen phosphate

OD

Optical density

PCR

Polymerase chain reation

PHENIX

Python-based hierarchical environment for integrated

Xtallography

pH

Negative logarithm of $\left[\mathrm{H}^{+}\right]$

$\mathbf{p} K_{a}$

Negative logarithm of the acid constant

PMSF

Phenylmethylsulfonyl fluoride

rpm

revolutions per minute

SDS

Sodium dodecyl sulfate

SDS-PAGE

Sodium dodecyl sulfate polyacrylamide gel electrophoresis

TAE

Tris-acetate-EDTA

TEMED

Tetramethylethylenediamine

TRH

Thyrotropin-releasing hormone

Tris

Tris(hydroxymethyl)aminomethane

$\mathrm{UV} / \mathrm{Vis}$

Ultraviolet visible spectroscopy

$V_{\max }$

Maximum reaction rate in $\mathrm{U} / \mathrm{mg}$ 
$\mathbf{w} / \mathbf{v} \quad$ Volume per volume

$\mathbf{w} / \mathbf{v} \quad$ Weight per volume

$X D S$

X-ray detector software

$\mathrm{Zn}$

Zinc

$\Delta \mathbf{H}$

Change in enthalpy

$\Delta \mathbf{S}$

Change in entropy

$\varepsilon$

Molar extinction coefficient in $\mathrm{M}^{-1} \mathrm{~cm}^{-1}$ 



\section{Introduction}

\subsection{Pyroglutamic Acid - A Key Metabolite Within the Human System}

All mono-/multi-cellular organisms in the kingdom of life undergo a multitude of important cellular processes like energy production and storage, cell respiration, cell transport and intercellular interactions. This great variety of cellular processes needs to be regulated by essential mechanisms in order to respond to external environmental influences and to maintain homeostasis. One mechanism for regulation of cellular activity are posttranslational modifications, which include covalent, enzymatic modifications of proteins and peptide species during or after protein biosynthesis. Some of the proteins and peptide species are directly modified at their amino acid side chains or at the corresponding Cor $\mathrm{N}$ - termini during protein biosynthesis, while other species are post-translationally modified after transport to a specific cell organelle or even outside the producing cell. Po-

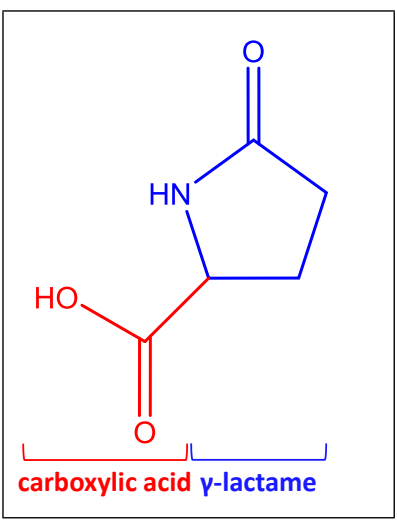

Figure 4.1: Chemical structure of pyroglutamic acid (5-oxo-L-proline). ssible post-translational modifications are for example proteolytic cleavage of regulatory subunits of proteins, covalent linkage of organic or inorganic functional groups, addition of lipids and modification of amino acids. As a result of a posttranslational modification the bioactivity of a certain protein or peptide species is drastically altered. One example for a post-translational modification of amino acids is the formation of pyroglutamic acid (5-oxo-L-proline) (Fig. 4.1) from glutamine or glutamic acid. Pyroglutamic acid was first discovered in 1882 by heating a glutamic acid containing solution up to $180{ }^{\circ} \mathrm{C}$, leading to an accelerated spontaneous cyclization reaction of the amino acid to pyroglutamic acid by the loss of one molecule of water [1]. Pyroglutamic acid is an essential compound within living cells and is formed by degradation of glutathione, spontaneous cyclization of activated glutamic acid during glutathione biosynthesis and by degradation of proteins containing pyroglutamic acid at the N-terminus [2]. It was reported that many proteins or small peptides, especially neuropeptides, contain an N-terminal pyroglutaminyl residue [3]. The physiological role of this modification is to increase protein resistance from degradation by aminopeptidases, thereby extending the half-life of a given peptide species or mediating receptor binding, 
which is important for cell signaling for instance [4]. In literature it was described that especially human neuropeptides like thyrotropin releasing hormone (TRH), neurotensin and orexin A harbor an N-terminal pyroglutamyl residue [2]. For decades it was believed that formation of pyroglutamic acid at the N-terminus of proteins and peptide species results from spontaneous cyclization of N-terminal glutamic acids $\left(k_{\text {non }}=1.0 \pm 0.4 \times\right.$ $\left.10^{-9} \mathrm{~s}^{-1}[5]\right)$ and glutamines $\left(k_{\text {non }}=1.7 \pm 0.2 \times 10^{-6} \mathrm{~s}^{-1}[5]\right)$ until the enzymatic formation was firstly described in the year 1963 [5]. This observation of an enzymatically driven pyroglutamic acid formation led to the focus on that enzyme class to decipher its reaction mechanism and its physiological importance in the post-translational processing of peptides and protein species.

\subsection{Glutaminyl Cyclase - An Enzyme Responsible For Pyroglutamic Acid Formation and Its Evolution}

The first described enzyme that was capable to catalyze the intramolecular cyclization reaction based on an N-terminal glutamine to pyroglutamic acid was isolated from the latex of the tropical plant Carica papaya and named glutaminyl cyclase [6]. The analogous enzyme of animal origin was later identified in the pituitary of mammals with the same catalytic proficiency $[7,8]$. Shortly after demonstrating an occurrence of glutaminyl cyclase in mammals the primary structure of the enzyme from Homo sapiens and Bos primigenius taurus was elucidated $[9,10]$. The physiological role of glutaminyl cyclases from mammals was reported to modify neuropeptides like hormones at their N-terminus post-translationally by catalyzing the conversion of an N-terminal glutamine to pyroglutamic acid. In addition, identification of a high structural homology of human glutaminyl cyclase (hQC) to bacterial zinc-dependent aminopeptidases resulted in the first impression of the tertiary structure of the enzyme [11]. The most accurately studied enzyme of this family is the leucine aminopeptidase from the marine bacterium Aeromonas proteolytica (APAP) [12-14], which in turn was used to model the tertiary structure of hQC in silico based on its sequence and structure [15]. Aminopeptidases specifically cleave the N-terminal residue from polypeptide chains and are involved in the metabolism within a living cell. However, tertiary structure of the monomeric leucine aminopeptidase determined by X-ray crystallography is characterized by a twisted eight-stranded $\beta$-sheet consisting of partly parallel and antiparallel $\beta$-sheets (Fig. 4.2 A) [16]. Additionally, the 
central $\beta$-sheet is surrounded by a number of $\alpha$-helices, which is a typical characteristic of proteins of the zinc-hydrolase superfamily [17]. Moreover, the active site of the leucine aminopeptidase is located at the protein surface in a cavity including two coordinated zinc ions. Zinc ions are coordinated by five highly conserved residues (His-Asp-Glu-Asp-His) and a water molecule, which acts as a bridging ligand, thereby connecting both metal ions.

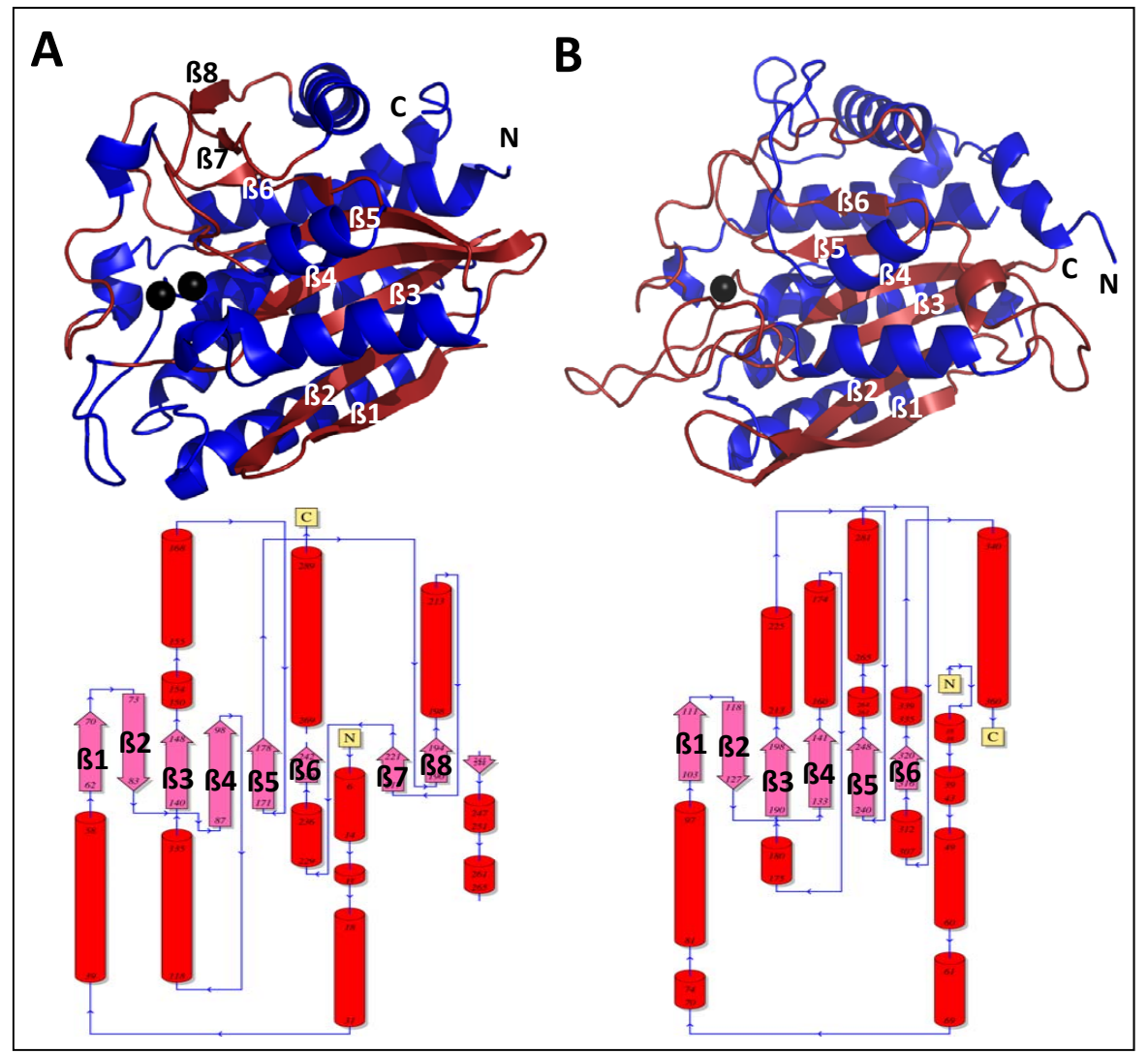

Figure 4.2: Comparison of the tertiary structure of human glutaminyl cyclase and leucine aminopeptidase of Aeromonas proteolytica. (A) Crystal structure of APAP (PDB entry: 1RTQ) and (B) hQC. $\beta$-sheets are depicted in red and $\alpha$-helices in blue. Zinc ions are represented as black spheres. Under the respective crystal structure the corresponding topology diagram was added. Amino-/Carboxyterminus and $\beta$-sheets are marked for each protein structure, respectively. Topology maps were generated with the web server PDBsum [18].

Validity of the in silico determined tertiary structure of hQC was later confirmed by X-ray crystallography $[19,20]$ and comparison of both crystal structures of hQC and APAP verified the proposed homology. The tertiary structure of hQC contains a central twisted six-stranded $\beta$-sheet, which forms the hydrophobic core of the protein, as it has been observed in the case of APAP (Fig. 4.2 B). Again, the central twisted $\beta$-sheet of hQC includes of two antiparallel and four parallel $\beta$-strands surrounded by ten $\alpha$-helices. These typical characteristics highlighted the affiliation of hQC to the metallopeptidase protein superfamily with the exception to catalyze different type of reactions. However, active 
site alignment of the crystal structures of APAP and hQC revealed a high conservation of the metal coordinating amino acid residues, but in case of hQC only one zinc ion was coordinated within the cavity at the protein surface (Fig. 4.3). Coordinating external ligands of the metal complex within the active site of hQC involves the residues His330, Asp159 and Glu202, while the fourth coordination site was occupied by a water molecule. This fact was quite surprising because the active site of hQC could provide the necessary coordinating amino acid residues to coordinate hypothetical two zinc ions. Therefore, it was assumed that the remaining amino acid residues adopted new functional tasks during evolution.

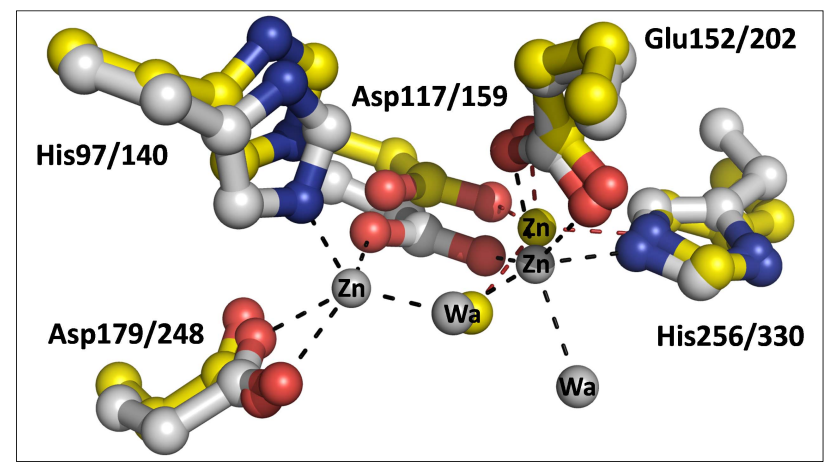

Figure 4.3: Active site alignment of APAP and hQC. The acitve site of APAP is shown in grey, whereas the active site of $\mathrm{hQC}$ is represented in yellow. Zinc ions $(\mathrm{Zn})$ and water molecules (Wa) are depicted in the corresponding colour of the respective active site. Dashed lines indicate coordination bonds $(\mathrm{APAP}=$ black, $\mathrm{hQC}=$ red). Amino acid residues and their corresponding positions are named respectively, in which the first number represents the amino acid position in APAP and the second one in hQC. Presented structure of APAP was modeled from PDB entry 1AMP.

Another indication of a structural relationship of hQC with zinc-dependent peptidases was the identification of an intramolecular disulfide bond [21]. The hQC as well as the APAP have in their primary structure two highly conserved cysteine residues, which form the disulfide bond at oxidizing conditions. In the case of APAP, the disulfide bond is involved in the formation of the active site, in which an interaction with the first amino acid side chain of the substrate occurs. As a result of the formed disulfide bond, enzymatic specificity of APAP is effected and additionally the structural integrity of the active site [16]. Analysis of both crystal structures showed that the disulfide bond is located close to the protein surface in case of APAP, while for hQC it is located near the hydrophobic core of the protein. Due to the location of the disulfide bond in hQC it was assumed that it is not directly involved in the formation of a catalytically active structure. This was demonstrated by kinetic experiments with hQC and a disulfide deficient variant, in which both enzyme types showed same catalytic efficiency [22]. Experimental data of thermal and chemical unfolding of hQC and the disulfide deficient variant demonstrated differences in their corresponding transition curves, suggesting a stabilizing role for the disulfide bond 
in terms of conformational stability.

However, one of the most important experimental evidences for an evolutionary relationship of hQC with APAP was the identification of a metal-dependency for catalysis [23]. The requirement of the zinc ion for hQC activity was displayed by time-dependent incubation of the enzyme with the heterocyclic chelator 1,10-phenanthroline [23]. Removal of the zinc ion from the active site resulted in inactive hQC, indicating the importance of the metal ion for N-terminal pyroglutamic acid formation catalyzed by hQC. This context makes it extremely likely that animal glutaminyl cyclase evolutionarily derived from a bacterial peptidase.

In addition, MALDI-TOF mass spectrometry and crystal structure of recombinant glutaminyl cyclase expressed in Pichia pastoris confirmed an N-glycosylation site (Asn49), which is located at the protein surface $[21,22]$. Glycosylated hQC has a molecular mass of $42.8 \mathrm{kDa}$, while complete deglycosylation with endoglycosidase $\mathrm{H}_{f}$ resulted in a molecular mass of $38.8 \mathrm{kDa}$ [21]. Apparently, the structural nature of the protein was not influenced by the cleavage of the oligosaccharides and did not affect enzyme catalysis. This was further confirmed by the expression of enzymatically active hQC in Escherichia coli, in which no glycosylation event occured $[11,21]$. Thus, it was suggested that lacking the glycosylation event could lead to a reduced solubility of the protein [22].

\subsection{Physiological Function of Mammalian Glutaminyl Cyclase}

It was suggested that the ubiquitous presence of glutaminyl cyclase in the animal kingdom could be important in various regulatory processes, for instance, cell signaling. Numerous regulatory peptides have already been isolated from animal tissues, which harbor an N-terminal pyroglutamyl residue $[24,25]$ probably caused by a post-translational modification. First indications of an enzymatically driven formation of N-terminal pyroglutamic acid were observed by mixing N-terminal glutaminyl peptides with extracts from bovine pituitary [8]. For this study, gonadotropin-releasing hormone (GnRH) and thyrotropinreleasing hormone $(\mathrm{TRH})$ were used as N-terminal glutaminyl peptides, harboring an Nterminal pyroglutamyl residue after incubation with extracts from bovine pituitary. TRH acts as a neurotransmitter and modulator of the central nervous system and triggers syn- 
thesis and release of thyrotropin in the hypophysis, while GnRH stimulates secretion of gonadotropins, which is important for male and female sexual functionality [26, 27]. In addition, northern blot analysis of glutaminyl cyclase mRNA distribution in bovine pituitary revealed high expression levels of the enzyme in brain and peripheral endocrine and exocrine glands [10]. Furthermore, it was postulated that the post-translational cyclization of glutamines at the N-terminus of small peptides presumably occurs within the regulated secretory pathway [28]. A possible post-translational modification within the regulated secretory pathway was indicated by a co-localization of glutaminyl cyclase with TRH and GnRH in secretory vesicles in the pituitary and hypothalamus. The cellular localization of the enzyme and the putative substrates in the regulated secretory pathway suggesting a role of glutaminyl cyclase in the synthesis of various hormones and neuropeptides, thereby directly involved in hormone maturation.

It was shown that biological activity of some hormones and neuropeptides depends on an N-terminal pyroglutamyl residue. An unmodified glutamine at the N-terminus of these hormones and neuropeptides results in a loss of binding affinity to their specific receptors, which would consequently interrupt cellular communication [24, 29]. Besides the importance of pyroglutamic acid at the first N-terminal amino acid position of some neuropeptides to develop an active conformation for receptor binding, it increases hormone stability. Due to the pyroglutaminyl-lactam ring the N-terminus loses its alkalinity, which is an essential feature for many aminopeptidases to recognize potential substrates. Therefore, the N-terminus of hormones and neuropeptides becomes resistant to non-specific proteolysis. Thus, bioactivity of the peptides is drastically enhanced by increasing their half-life. In summary, involvement of glutaminyl cyclase in the synthesis of a variety of peptides with regulatory function allocates the enzyme an essential role in hormone metabolism.

\subsection{Glutaminyl Cyclase And Its Role in Neurodegenerative Diseases}

Although the physiological function of pyroglutamic acid and glutaminyl cyclase seems to be essential for generation of biologically active structures of hormones and neuropeptides, several reports demonstrated that formation of pyroglutamic acid is also connected with pathophysiological processes, especially in amyloidogenic diseases (Fig. 4.4) [30]. In this context, Alzheimer's disease (AD) is perhaps the best known amyloidogenic disease with 
great histopathological changes.

Alzheimer's disease is a progressive, neurodegenerative and, until today, an incurable disease, which occurs predominantly in patients of the middle and advanced age. The disease was described by the German physician Alois Alzheimer for the first time in 1906, until his observations and results were published in 1907 [31]. An autopsy of the brain of a deceased woman in middle age, who suffered from severe memory problems and confusion, was performed in which he identified dense deposits around the outside of nerve cells of the central nervous system. Worldwide, about 44 million people are suffering from typical symptoms of this disease, which includes memory loss, loss of speech and judgment, personality changes and mood swings [32].

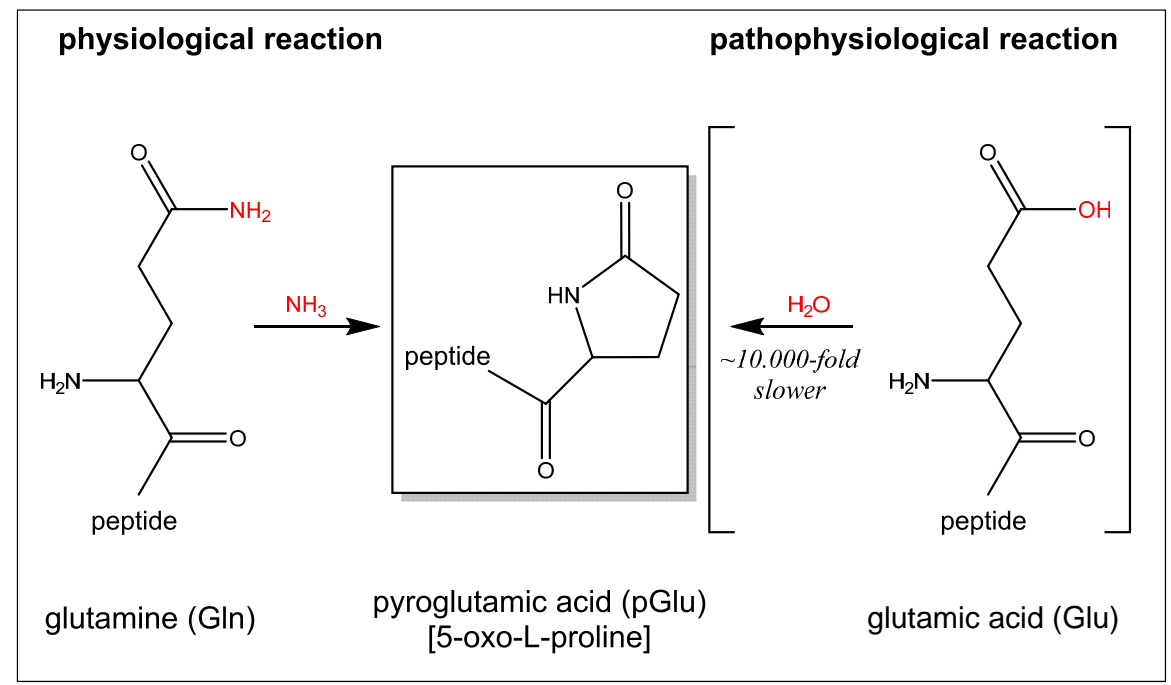

Figure 4.4: Overview of catalyzed reactions by hQC. N-terminal glutamine of small peptides are converted in an irreversible cyclization reaction to pyroglutamic acid (physiological reaction). In addition, the enzyme catalyzes in a pathophysiological side reaction also glutamic acid at the N-terminus of the processed $\mathrm{A} \beta$ peptide.

Brains of individuals with advanced symptoms are characterized by increased deposits of amyloid plaques in the neocortical regions of the brain [33]. Amyloid plaques mainly consist of beta-amyloid peptide species, which are formed by processing of the amyloid precursor protein (APP) (Fig. 4.5) [34]. APP is a highly conserved ubiquitous transmembrane protein, which is expressed in most mammalian tissues with the highest levels found in the nervous system and whose physiological function is not yet completely described. One reason could be that APP is a very heterogeneous molecular species, resulting from different splice variants of mRNA and various post-translational modifications like glyco- 
sylation and phosphorylation [35, 36]. During and after transport of the APP through the secretory pathway, the protein can be cleaved in different ways by $\alpha-/ \beta-/ \gamma$-secretase [37]. Proteolytic cleavage of the soluble extracellular part of the protein by $\alpha$-secretase results in a so-called C-83 fragment, which remains at the plasma membrane (non-amyloidogenic pathway) (Fig. 4.5) [38]. Another way of APP processing involves proteolytical cleavage by $\beta$ - and $\gamma$-secretase (amyloidogenic pathway). The proteolytic cleavage by $\beta$-secretase activity results in a slightly smaller secreted fragment (APP $\beta)$ and a larger transmembrane portion (C-99 fragment). The C-99 fragment is subsequently cleaved by $\gamma$-secretase activity within the transmembrane portion. A number of different potential proteolytic interfaces for $\gamma$-secretase activity results in various small amyloidogenic peptide species (A $\beta$ peptides), which differ in their sequence length. Furthermore, after proteolytic processing by $\beta$ - and $\gamma$-secretase each $\mathrm{A} \beta$ peptide harbors an $\mathrm{N}$-terminal glutamyl residue. Under normal circumstances these different $\mathrm{A} \beta$ peptides, whose physiological function is to date unknown, can be degraded proteolytically by exopeptidases. However, if the different A $\beta$ peptide species are co-localized with hQC the N-terminal glutamic acid can be converted to pyroglutamic acid.

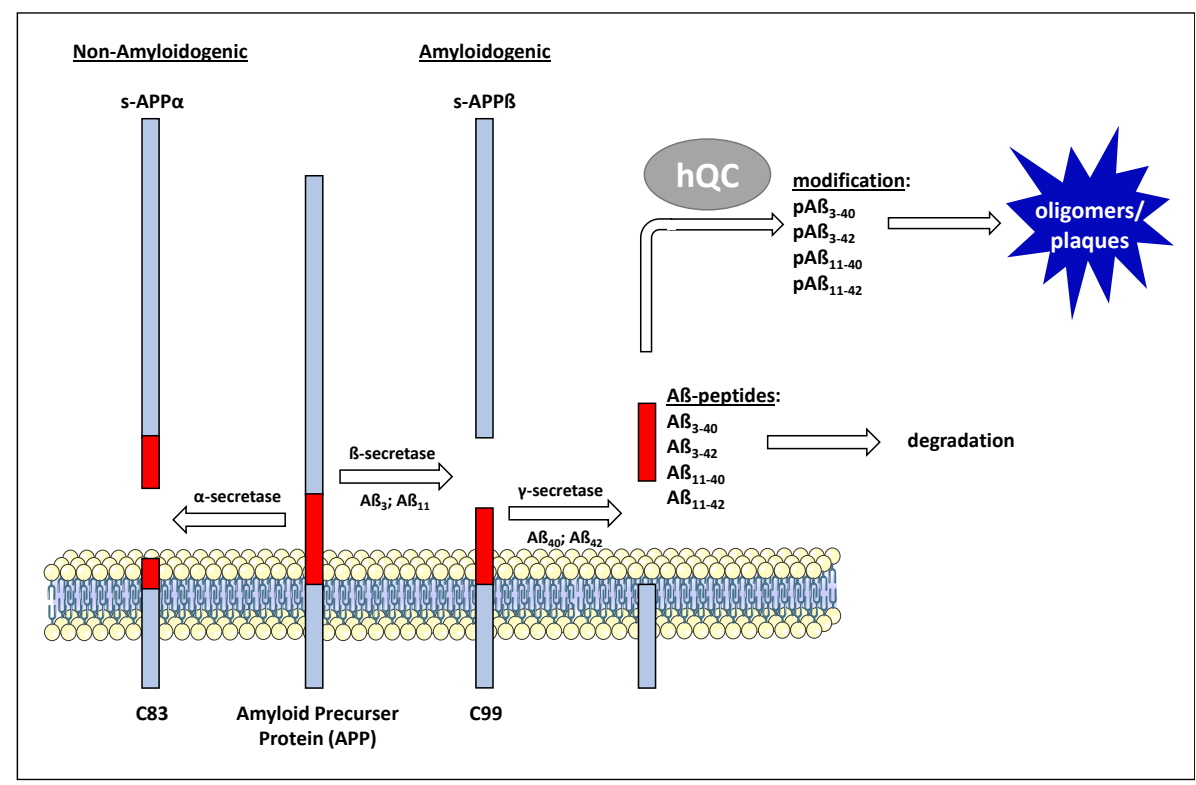

Figure 4.5: Schematic overview of the involvement of hQC during Alzheimer's disease development. The transmembrane APP gets processed by $\beta$ - $/ \gamma$-secretase to beta-amyloid peptides varying in length, but each peptide species harbors an N-terminal glutamic acid. Once there is a co-localization of the beta-amyloid peptides and hQC the N-terminal glutamic acid gets converted to pyroglutamic acid.

For a long time it was believed that unmodified $\mathrm{A} \beta$ peptides have a tendency to aggregate [39] and that an increased formation of the peptides is associated with accelerated progres- 
sion of the Alzheimer's disease [40, 41]. In later studies, the presence of a pyroglutamyl residue was described at the $\mathrm{N}$-terminally truncated $\mathrm{A} \beta$ peptide species [30] and further proven that the majority of the deposited amyloidogenic plaques consists of the modified form $[42,43]$. Because of the relative high percentage of the pyroglutamyl modified $\mathrm{A} \beta$ peptides in the amyloidogenic plaques, it was concluded that these peptides could play a crucial role in the formation of the disease, especially in the initial phase [44]. Indeed, investigating the aggregation behavior of modified $\mathrm{A} \beta$ peptide species showed that these were more prone to aggregation than unmodified $\mathrm{A} \beta$ peptide species [45]. One possible explanation is that the solubility of the pyroglutamyl modified $\mathrm{A} \beta$ peptide is drastically decreased at physiological $\mathrm{pH}$ compared to unmodified $\mathrm{A} \beta$ peptide species, which, in turn, increases the aggregation propensity $[46,47]$. Due to these properties and the dominant presence of modified amyloid peptides in amyloidogenic plaques, it is presumed that these molecular species could form the initial deposits and therefore accelerates the emergence and progression of the disease [44, 48].

In addition to the enhanced aggregation and toxicity of modified $\mathrm{A} \beta$ peptide due to the N-terminal pyroglutamyl residue, it is believed that the modification of the N-terminus leads to an increased stability to proteolytic degradation [49]. First cell culture experiments with modified and unmodified $\mathrm{A} \beta$ peptide species showed a reduced degradation of A $\beta$ peptide species harboring an N-terminal pyroglutamyl residue [50]. Moreover, recent works demonstrated that different proteolytic enzyme activities, such as pyroglutamyl aminopeptidase and glutamyl aminopeptidase, decrease with age [51, 52]. This would in turn mean a slowed degradation of modified and unmodified $\mathrm{A} \beta$ peptide species. One possible explanation of the origin of Alzheimer's disease is a general dysfunction of the N-terminal degradation of unmodified $\mathrm{A} \beta$ peptide species by aminopeptidases [49]. Due to the reduced degradation of the $\mathrm{A} \beta$ peptide species an increased concentration of intermediates for aggregation arise, leading finally to the toxic amyloidogenic plaques.

However, it is still unclear how the modification of N-terminal glutamic acid to pyroglutamic acid occurs in vivo. After proteolytic cleavage of the APP by $\beta$ - and $\gamma$-secretase each $\mathrm{A} \beta$ peptide species harbor a glutamyl residue at its $\mathrm{N}$-terminus. Unlike pyroglutamyl formation of N-terminal glutamine catalyzed by hQC, enzymatic cyclization of N-terminal 
glutamic acid at physiological conditions are not described so far. Therefore it was concluded that pyroglutamyl formation of N-terminal glutamyl residues occurs spontaneously until various derivatives of glutamic acid were tested in presence/absence of hQC [3]. It was shown that N-terminal glutamyl residues were not prone to spontaneous cyclization, but in presence of hQC the N-terminal pyroglutamyl residue was detected, indicating a specific conversion catalyzed by the enzyme. An inhibition of the hQC would lead to a decreased formation of modified $\mathrm{A} \beta$ peptide species and could therefore serve as a way to slow down the manifestation of Alzheimer's disease.

\subsection{Reaction Mechanism of Human Glutaminyl Cyclase}

The elucidation of the reaction mechanism of enzyme catalysis is an essential prerequisite for the development of potent inhibitors. Structures for potential enzyme inhibitors could be derived from intermediates during enzyme catalysis or already optimized inhibitor structures. The hQC is, due to their physiological and possibly pathophysiological function, a potential target for enzyme drug development. Therefore, the understanding of the catalytic mechanism of this enzyme is of great importance.

Two possible mechanisms of hQC catalysis were predicted in the past, which fundamentally differed in their modes of action [53]. First, the catalysis of the formation of a covalent intermediate (acyl-enzyme intermediate) could occur. In this mechanism a nucleophilic residue of the active site, for example an activated cysteine residue, attacks the $\gamma$-amide group of the N-terminal glutamine of the substrate. The acyl-enzyme intermediate is formed and ammonia is released (acylation). In a second step, the $\alpha$-amino group of glutamine attacks nucleophilically the $\gamma$-carbonyl. The product is released and the initial state is resumed (deacylation). Catalysis of hQC would be in this case in accordance with the mechanism of catalysis of serine/cysteine proteases or $\gamma$-glutamyl cyclotransferases. The second alternative mechanism is based on a non-covalent reaction catalyzed by hQC (Fig. 4.6). Due to an intramolecular nucleophilic attack of the $\alpha$-amino group at the $\gamma$-carbonyl of an N-terminal glutamine the substrate forms a tetrahedral, non-covalently enzyme-bound intermediate, which decomposes into ammonia and the pyroglutamyl peptide. Accordingly, hQC would exercise the catalytic function by binding of the substrate, which promotes a nucleophilic attack, and the stabilization of the tetrahedral intermediate. 
Initial studies on hQC from pig pituitary suggested that the enzyme might possess thiol groups which are essential for catalysis [7]. However, identification of a disulfide bridge in hQC lead to exclusion of covalent catalysis with participation of cysteine residues [21]. In addition, glutaminyl cyclases of mammals showed no inhibition by serine modifying inhibitors, which supports the idea of a non-covalent catalysis [53].



Figure 4.6: Catalytic cycle of hQC catalyzing N-terminal glutaminyl substrates. First step is the formation of the Michaelis-Menten complex via binding of the substrate. Thereby it displaces the coordinated water molecule and occupies the fourth coordination site of the catalytic zinc. The catalytic zinc ion acts as a Lewis acid, pulls out electrons from the $\gamma$-carbonyl moiety of the N-terminal glutamine, thereby activating the $\gamma$-carbonyl carbon electrophilically. In addition, Glu201 activates via acid-base catalysis the $\alpha$-amino group, which in turn gets more nucleophilic. Afterwards, the $\alpha$-amino group performs a nucleophilic attack on the $\gamma$-carbonyl carbon, leading to a short-lived tetrahedral intermediate. Next, an intrinsic proton transfer to the potential leaving group via a conserved hydrogen bond network is performed to subsequently release ammonia and the product.

The enzyme catalyzes the stereospecifical cyclization of N-terminal glutaminyl residues in its physiological reaction and exhibits high selectivity for substrates with aromatic amino acids in the second N-terminal amino acid position [54]. Moreover, hQC showed a pHdependence for N-terminal glutaminyl substrates, which are preferentially converted at 
alkaline $\mathrm{pH}$-values, especially at $\mathrm{pH} 8.0$ [15]. It was shown in steady-state pH-dependency experiments that the Michaelis-Menten constant $\left(K_{\mathrm{M}}\right)$ was influenced at $\mathrm{pH}$-values above 8.0, indicating that hQC can bind the substrate only in the N-terminal deprotonated state. The $\mathrm{pH}$-dependence of the catalytic activity depends therefore on the protonation state of the $\alpha$-amino group of the substrate.

Studies of the substrate specificity of hQC and its structural relationship to the zincdependent aminopeptidase family led to the first hypothesis of the mechanism of catalysis and the role of the metal ion [54]. The related zinc-dependent aminopeptidase has two metal ions within their active site, which resumes different tasks during catalysis. For instance, to polarize the scissile peptide bond by interacting with the carbonyl oxygen or to increase the nucleophilicity of the attacking water molecule. Most importantly, binding of the oxanion to stabilize the tetrahedral intermediate, which resulted from the nucleophilic attack. In the case of aminopeptidase from APAP, which has the highest structural homology to the animal glutaminyl cyclases, these tasks are performed mainly by one zinc ion in the active site, while the other metal ion is only used to fix the N-terminal amino nitrogen during catalysis. However, pyroglutamyl formation catalyzed by hQC differs compared to the mechanism of APAP. First, in contrast to the hydrolysis reaction of APAP, which removes the first N-terminal amino acid residue, the cyclization reaction of hQC is an intramolecular reaction. Second, the activation of the attacking nucleophile, which is an essential part in the catalytic cycle of APAP, is not necessary for hQC, because the free N-terminal nitrogen has a strong nucleophilicity. Despite these differences, partial reaction steps of the catalytic mechanisms of APAP and hQC could be the same. For example, in both cases the reaction is an addition elimination mechanism $\left(\mathrm{S}_{N} 2_{t}\right)$. A zinc ion in the active site of hQC could therefore polarize the $\gamma$-carbonyl group of the substrate to increase the electrophilicity of the carbonyl carbon and, additionally, stabilize the oxanion interaction of the resulting tetrahedral intermediate after the nucleophilic attack of the $\alpha$-amino group.

Crystal structures of APAP clearly showed two coordinated zinc ions in the active site, whereas for hQC only one zinc ion was identified even though the coordinating amino acid residues are highly conserved in both enzymes [16, 20]. As already mentioned, it 
was assumed that the non-coordinating amino acids in hQC adopted new functions during evolution. This was demonstrated by site-directed mutagenesis experiments, in which substitution resulted in a non-functional enzyme (Asp248) or reduced catalytic activity (His140) [55]. Due to the position of Asp248 in the crystal structure it was concluded that it forms together with Glu201 and Asp305 a conserved hydrogen bond network, which is crucial for proton shuttling from the $\alpha$-amine group of the tetrahedral intermediate to the $\gamma$-amide amino group (Fig. 4.6).

Nevertheless, a detailed reconstruction of the catalytic steps of the reaction is not yet possible. For instance, the short-lived tetrahedral intermediate is only postulated, but so far not experimentally confirmed.

\subsection{Human Glutaminyl Cyclase - Target Enzyme For Drug Development}

Involvement of hQC activity in the formation of pyroglutamyl peptides and its essential role in the initial phase of Alzheimer's disease makes it a potential candidate for pharmacological drug development. The primary interest of the development of glutaminyl cyclase inhibitors are agents suppressing the formation of toxic pyroglutamyl peptides at the N-terminus of $\mathrm{A} \beta$ peptides. By the inhibition of the glutaminyl cyclase activity in neuronal cells of the central nervous system of Alzheimer's disease patients would presumably prevent formation of N-terminal glutamyl cyclization. Thus, the $\mathrm{A} \beta$ peptide species would be unprotected against proteolysis by aminopeptidases. Accordingly, an increased degradation of the peptides would be the result, which in turn would contribute to decreased plaque formation.

Different attempts were performed with compounds which were described in literature to form a complex with metal ions to inhibit hQC activity competitively. It was shown that compounds especially on imidazole-based structure are promising interaction partners for the zinc ion within the active site of hQC $[23,56]$. As a consequence, different imidazole derivatives were successfully tested as competitive inhibitors for hQC. Although, these compounds still need to be investigated and optimized in terms of their toxicity, the inhibitory potency and bioavailability. 


\subsection{Motivation}

Due to the fact that all described inhibitors for hQC are imidazole-based, which is a common metal chelator, further usage could led to off-target inhibition of other metalloenzymes within the human metabolism. This interference with other metal-dependent enzymes, like dipetidyl peptidase 4, might cause severe side effects. Therefore, the longterm goal of this field of investigation is the development of enzyme specific and mechanism based hQC inhibitors. If possible, the potential inhibitor should adopt a molecular structure which mimics a reaction intermediate or transition state in the trajectory of hQC catalysis. A transition state intermediate during hQC catalysis would be the postulated tetrahedral intermediate. This tetrahedral transition state intermediate is highly energetic, short-lived and generally reacts to further intermediate states. Specific binding of inhibitors to hQC might be achieved by mimicking sterical and conformational features of the reaction intermediate or a transition state. Strong competitive binding of the inhibitor to hQC should drastically reduce turnover of hQC, especially formation of pyroglutamyl modified $\mathrm{A} \beta$ peptides.

Another part of this work is the elucidation of the physiological (glutaminyl) and pathophysiological (glutamyl) reaction of hQC. To obtain information about catalytic efficiencies of the physiological and pathophysiological reaction, glutaminyl-/glutamyl substrates catalyzed by hQC need to be kinetically characterized. In addition, elucidation of the binding mode of the physiological and pathophysiological substrates should give deeper insight in the catalytic variability of hQC. These investigations should clarify how the enzyme mechanistically accomplishes to catalyze two different substrates. 


\section{Materials and Methods}

\subsection{Materials}

\subsubsection{Chemicals}

Compound

Acrylamide (30 \%)

Acetic acid

Agar

Agarose low EEO

Ammonium peroxodisulfate

Ammonium sulfate

Adenosine triphosphate

Bromophenol blue sodium salt

Carbenicillin disodium salt

Chelex-100 Resin

Cobalt(II)-chloride

Coomassie-Brilliant blue G-250

Disodium hydrogen phosphate

DTT

EDTA

Ethanol

Ethidium bromide

Ethylene glycol

Glycerol anhydrous

Hellmanex

Hydrochloric acid (37 \%)

Imidazole

IPTG

Isopropanol

L-Glutathione reduced

Magnesium sulfate

Magnesium chloride hexahydrate
Supplier

Carl Roth GmbH \& Co KG, Karlsruhe Carl Roth GmbH \& Co KG, Karlsruhe AppliChem GmbH, Darmstadt AppliChem GmbH, Darmstadt

Carl Roth GmbH \& Co KG, Karlsruhe Carl Roth GmbH \& Co KG, Karlsruhe Carl Roth GmbH \& Co KG, Karlsruhe AppliChem GmbH, Darmstadt

Carl Roth GmbH \& Co KG, Karlsruhe Bio-Rad Laboratories, Inc., USA

Sigma-Aldrich, Steinheim

AppliChem GmbH, Darmstadt

Carl Roth GmbH \& Co KG, Karlsruhe AppliChem GmbH, Darmstadt AppliChem GmbH, Darmstadt Sigma-Aldrich, Steinheim Carl Roth GmbH \& Co KG, Karlsruhe AppliChem GmbH, Darmstadt AppliChem GmbH, Darmstadt Sigma-Aldrich GmbH, Steinheim Carl Roth GmbH \& Co KG, Karlsruhe AppliChem GmbH, Darmstadt AppliChem GmbH, Darmstadt AppliChem GmbH, Darmstadt Carl Roth GmbH \& Co KG, Karlsruhe Carl Roth GmbH \& Co KG, Karlsruhe Carl Roth GmbH \& Co KG, Karlsruhe 
MES

$\mathrm{NADH}$

Naphtalene-2-sulfonamide

Phenyl phosphorodiamidate

Peptone/tryptone

PMSF

Polyethylene glycol 400

SDS

Sodium chloride

Sodium dihydrogen phosphate dihydrate

Sodium hydroxide

TEMED

Tris ultrapure

Yeast extract

Zinc sulfate

$\alpha$-Ketoglutaric acid disodium salt

1,4-Dioxane

1,10-phenanthroline monohydrate

2-Mercaptoethanol

\subsubsection{Enzymes, Markers and Kit systems}

Enzymes, Markers and Kit systems

DNase I

dNTP mix $(10 \mathrm{mM})$

$D p n \mathrm{I}(10 \mathrm{U} / \mu \mathrm{l})$

Gene Ruler ${ }^{T M} 1 \mathrm{~kb}$ DNA ladder $0,5 \mu \mathrm{g} / \mu \mathrm{l}$

L-Glutamic dehydrogenase from bovine liver Lysozyme $(25000 \mathrm{U} / \mathrm{mg})$

$\operatorname{Not} \mathrm{I}(10 \mathrm{U} / \mu \mathrm{l})$

NucleoSpin ${ }^{\circledR}$ Gel and PCR Clean-up

NucleoSpin ${ }^{\circledR}$ Plasmid
AppliChem GmbH, Darmstadt

Sigma-Aldrich GmbH, Steinheim

Sigma-Aldrich GmbH, Steinheim

Sigma-Aldrich GmbH, Steinheim

AppliChem GmbH, Darmstadt

AppliChem GmbH, Darmstadt

AppliChem GmbH, Darmstadt

AppliChem GmbH, Darmstadt

AppliChem GmbH, Darmstadt

Carl Roth GmbH \& Co KG, Karlsruhe

AppliChem GmbH, Darmstadt

Carl Roth GmbH \& Co KG, Karlsruhe

AppliChem GmbH, Darmstadt

AppliChem GmbH, Darmstadt

AppliChem GmbH, Darmstadt

Sigma-Aldrich, Steinheim

Sigma-Aldrich, Steinheim

Carl Roth GmbH

Carl Roth GmbH \& Co KG, Karlsruhe

Supplier

AppliChem GmbH, Darmstadt

MBI Fermentas, St. Leon Rot

Thermo Scientific GmbH, Schwerte

Fermentas GmbH, St.Leon-Rot

Sigma-Aldrich, Steinheim

AppliChem GmbH, Darmstadt

Thermo Scientific GmbH, Schwerte

Macherey-Nagel GmbH \& Co. KG, Düren

Macherey-Nagel GmbH \& Co. KG, Düren 
Phusion Green High-Fidelity DNA Poly- Thermo Scientific GmbH, Schwerte merase $(2 \mathrm{U} / \mu \mathrm{l})$

PreScission Protease

$\operatorname{Sal\mathrm {I}}(10 \mathrm{U} / \mu \mathrm{l})$

T4 DNA Ligase $(5 \mathrm{U} / \mu \mathrm{l})$

Unstained protein molecular weight standard

6x DNA Loading Dye
Self-expressed and purified

Thermo Scientific GmbH, Schwerte

Thermo Scientific GmbH, Schwerte

Fermentas GmbH, St.Leon-Rot

Fermentas GmbH, St.Leon-Rot

\subsubsection{Peptides}

Peptides

Gln-Gln (QQ)

Ala-Phe-Ala (AFA)

Glu-Phe-Arg-His (EFRH)

Gln-Phe-Arg-His (QFRH)

pGlu-Phe-Arg-His (pEFRH)

Gln-Leu-Tyr-Glu-Asn (QLYEN) (Neurotensin ${ }_{1-5}$ )

Gln-His-Trp-Ser-Tyr (QHWSY) $\left(\mathrm{GnRH}_{1-5}\right)$

Gln-Pro-Leu-Pro-Asp (QPLPD) (OrexinA $\left.A_{1-5}\right)$

Glu( $\gamma$-hydrazide)-Phe-Ala (E( $\gamma$-hydrazide)FA) $\underline{\text { Supplier }}$

Innovagen $\mathrm{AB}$, Lund

Innovagen $\mathrm{AB}$, Lund

Innovagen $\mathrm{AB}$, Lund

Innovagen $\mathrm{AB}$, Lund

Innovagen $\mathrm{AB}$, Lund

Innovagen $\mathrm{AB}$, Lund

Innovagen $\mathrm{AB}$, Lund

Innovagen AB, Lund

Fraunhofer Institute, Halle

\subsubsection{Bacterial Strains}

Bacterial strain

Genotype

$\underline{\text { Supplier }}$

E.coli $\mathrm{DH} 5 \alpha$

$\mathrm{F}^{-}$\$80lacZ $\Delta \mathrm{M} 15 \Delta($ lacZY $\arg \mathrm{F}) \mathrm{U} 169$ recA1 end $\mathrm{A} 1$ Invitrogen, hsdR17 $\left(\mathrm{r}_{K}^{-} \mathrm{m}_{K}^{+}\right)$phoA supE44 $\lambda^{-}$thi-1 gyr A96 relA1 Karlsruhe

E.coli XL1-blue $\mathrm{F}^{\prime}$ recA1 endA1 gyr A96 thi-1 hsdR17 supE44 relA1 Stratagen, lac $\left[\right.$ proAB $\left.l a c I^{q} \mathrm{Z} \Delta \mathrm{M} 15 \operatorname{Tn} 10\left(\operatorname{Tet}^{R}\right)\right] \quad$ Heidelberg

E.coli BL21 Star $\mathrm{F}^{-}$omp $\mathrm{T} d c m$ hsd $\mathrm{S}\left(\mathrm{r}_{B}^{-} \mathrm{m}_{B}^{-}\right)$gal $\left[\mathrm{malB}^{+}\right]_{K-12}\left(\lambda^{S}\right) \quad$ Invitrogen, Karlsruhe 


\subsubsection{Oligonucleotides}

\begin{tabular}{|c|c|}
\hline Oligonucleotide & Sequence 5' $\rightarrow$ 3' \\
\hline $\mathrm{hQC}_{30-361}$ SalI for & ggccatgcgtcgactcAGTCCGAGTGCCTCAGCCTGGC \\
\hline $\mathrm{hQC}_{30-361}$ NodI rev & $\begin{array}{l}\text { CCTACAAGTCTTTGTGTTGGAATATCTTCATTTGTAA } \\
\text { gcggccgcgcatggcc }\end{array}$ \\
\hline $\mathrm{hQC}_{35-361}$ opti. rev & GGAAGTTCTGTTCCAGGGGCCC \\
\hline $\mathrm{hQC}_{35-361}$ opti. for & GCCTGGCCAGAGGAGAAGAATTACC \\
\hline Sequencing primer 1 for & GGGCCTTGTGCAACCCAC \\
\hline Sequencing primer $2 \mathrm{rev}$ & GCACAACATGTTGGGTGGTTG \\
\hline Sequencing primer 3 for & GAATTCATCGGCTCTTCG \\
\hline
\end{tabular}

\subsubsection{Plasmids}

Plasmid

pQE-31

pGEX-6P-1

pGEX-6P-1
Characteristics

Expression of SUMO-hQC $30-361$

Expression of PreScission Protease

Expression of GST-hQC $35-361$ $\underline{\text { Selection marker }}$

Carbenicillin $^{R}$

Carbenicillin $^{R}$

Carbenicillin $^{R}$

\subsubsection{Devices}

$\underline{\text { Device }}$

Absorbance Photomultiplier R928

Äkta Prime Plus

Äkta Purifier

Centrifuge Avanti HP-30 I (Rotor JA-30.50)

Circular dichroism spectropolarimeter

Conducting meter FiveEasy

Gel documentation system

Incubation shaker, Unitron

ITC 200 MicroCalorimeter

Microfluidizer 110S

NanoDrop2000
Supplier

Applied Photophysics, UK

GE Healthcare, Munich

GE Healthcare, Munich

Beckmann Coulter, Krefeld

Applied Photophysics Ltd., UK

Mettler-Toledo, Giessen

Herolab GmbH, Wiesloch

Infors AG, Switzerland

Microcal, Northampton, USA

Microfluidics, USA

Thermo Scientific, Schwerte 
pH-Meter 7110

SDS gel electrophoresis SE250

SX18 MV stopped-flow spectrometer

Thermocycler TProfessional

Thermomixer comfort

Unitron Plus AJ252 incubator

UV-Vis Spectrometer, V-650

X-ray MM-007 rotating-anode generator

X-stream ${ }^{T M} 2000$ Cryogenic Crystal Cooler

X-ray Mar 345dtb image plate detector
Inolab-WTW, Weilheim

Hoefer, Holliston, USA

Applied Photophysics, UK

Biometra, Göttingen

Eppendorf, Hamburg

Infors HT, Switzerland

Jasco GmbH, Groß-Umstadt

Rikagu Corp., USA

Rikagu Corp., USA

Rikagu Corp., USA

\subsubsection{Columns}

\section{Columns}

Glutathione Sepharose ${ }^{\circledR} 4$ FF $(16 / 10)$

Source 30Q (16/10)

HiPrep ${ }^{T M} 26 / 10$ Desalting
Supplier

GE Healthcare, Munich

GE Healthcare, Munich

GE Healthcare, Munich

\subsubsection{Commodities}

Commodities

CrystalCap HT cryoloop

CrystalCap HT vial

UV- and CD-cuvettes

Spin- ${ }^{\circledR}$ UF concentrator (30,000 MWCO)

$\operatorname{VDXm}^{T M}$ crystallization plates with sealant

$0.2 \mu \mathrm{m}$ Filter

$0.22 \mu \mathrm{m}$ PES

$18 \mathrm{~mm}$ Circular cover slides - siliconized $\underline{\text { Supplier }}$

Hampton Research, USA

Hampton Research, USA

Hellma, Müllheim

Corning, USA

Hampton Research, USA

Sartorius, Göttingen

Bio-Rad Laboratories, Inc., USA

Jena Bioscience GmbH, Jena 


\subsection{Methods}

\subsubsection{Molecular Biology}

\subsubsection{Polymerase Chain Reaction (PCR)}

PCR [57] was used for amplification of the QCPT gene from the pQE-31 vector as well as for cloning the gene of interest into the multiple cloning site of the pGEX-6P-1 vector. A standard PCR reaction contained 1x HF buffer, $200 \mu \mathrm{M}$ dNTP mix, 5-50 ng template DNA, 125 pmol 5'-phosphorylated sense and antisense primer and 1 U Phusion DNA Polymerase. In addition, PCR was also used to optimize the gene construct for crystallization by deletion of specific nucleic acids. In this case a standard PCR reaction contained $1 \mathrm{x}$ HF buffer, $200 \mu \mathrm{M}$ dNTP mix, 5 ng template DNA, 0.5 pmol 5'-phosphorylated sense and antisense primer and $1 \mathrm{U}$ Phusion DNA Polymerase. For the described PCR reactions the following temperature protocol was used (Tab. 5.1).

Table 5.1: Temperature protocol for PCR reaction. A denaturation, annealing and elongation step counts as one cycle, respectively. After each cycle the temperature increases by the specified $\Delta \mathrm{T}$ value. A final denaturation and final elongation step also counts as one cycle.

\begin{tabular}{l|c|c|c|c} 
Step & Temperature $\left[{ }^{\circ} \mathrm{C}\right]$ & Time $[\mathrm{s}]$ & Cycles & $\Delta \mathrm{T}\left[{ }^{\circ} \mathrm{C}\right]$ \\
\hline Initial denaturation & 98 & 30 & & \\
Denaturation & 98 & 10 & 10 & 0.5 \\
Annealing & 70 & 30 & 10 & 0.5 \\
Elongation & 72 & 75 & 10 & 0.5 \\
Final denaturation & 98 & 10 & 20 & \\
Final Elongation & 72 & 90 & 20 & \\
Storage & 4 & & &
\end{tabular}

\subsubsection{Cleavage of DNA with Restriction Enzymes}

For insertion of the QCPT gene into the multiple cloning site of the target vector, restriction enzymes NotI and SalI were used to digest the pGEX-6P-1 vector and insert. DNA restriction was performed according to the manufacturer's manual of the used endonucleases. 


\subsubsection{Agarose Gel Electrophoresis}

To analyze generated PCR products by size-dependent separation, horizontal agarose gel electrophoresis [58] was performed. During this study gels with an agarose content of $1 \%$ (w/v) in 1x TAE buffer (40 mM Tris, $1 \mathrm{mM}$ EDTA, $20 \mathrm{mM}$ acetate, $\mathrm{pH}$ 8.5) were used. Prior to gel electrophoresis, DNA samples were mixed with 6x loading dye and loaded onto the gel. After electrophoresis the gel was incubated in a $2 \mu \mathrm{g} / \mathrm{ml}$ ethidium bromide bath for 10 min. For visualization of the DNA ethidium bromide was excited at $\lambda=285 \mathrm{~nm}$ in a gel documentation system. To estimate separated DNA-fragments a DNA standard ladder was always co-electrophoresed.

\subsubsection{Blunt-End Ligation of DNA Fragments}

For ligation of two DNA fragments T4 DNA Ligase was used according to the manufacturer's manual. In addition, to stop the ligation reaction, it was incubated for $10 \mathrm{~min}$ at $65{ }^{\circ} \mathrm{C}$. Afterwards, $5 \mathrm{U}$ of $D p n \mathrm{I}$ were added and incubated over night at $37^{\circ} \mathrm{C}$ to digest the original methylated plasmid. To inactivate $D p n \mathrm{I}$, the reaction was incubated for $5 \mathrm{~min}$ at $80{ }^{\circ} \mathrm{C}$.

\subsubsection{Concentration and Purity Determination of DNA}

DNA concentrations were determined spectroscopically using a NanoDrop2000. The absoption at $260 \mathrm{~nm}$ was used with the following correlation to determine the concentrations:

$$
1 \mathrm{~A}_{260} \text { Unit of dsDNA } \widehat{=} 50 \mu \mathrm{g} / \mathrm{ml} \text { in } \mathrm{H}_{2} \mathrm{O}
$$

To guarantee a high purity grade the $\mathrm{A}_{260} / \mathrm{A}_{280}$ ratio should always be in a range of 1.8 $\leq \mathrm{A}_{260} / \mathrm{A}_{280} \leq 2.0$. An $\mathrm{A}_{260} / \mathrm{A}_{280}<1.8$ indicates a contamination with proteins and aromatic substances and an $\mathrm{A}_{260} / \mathrm{A}_{280}>2$ is an indicator for RNA contamination.

\subsubsection{Plasmid Transformation into Chemocompetent E.coli Cells}

Generation of chemical competent $E$. coli cells for transformation were prepared according 
to a standard protocol [59] and stored in $50 \mu \mathrm{l}$ aliquots at $-80{ }^{\circ} \mathrm{C}$ for several months. For transformation of the plasmid into chemocompetent E. coli cells 200-400 ng of the target vector DNA was added to the cells and gently mixed, followed by an incubation step for 20 min on ice. Next, cells were heat shocked at $42{ }^{\circ} \mathrm{C}$ for $60 \mathrm{~s}$ and subsequently cooled down on ice for 2 min. For a slow regeneration of the E.coli cells and to express the corresponding antibiotic resistance genes, $1 \mathrm{ml}$ of nonselective LB-media (10 g/L tryptone, $5 \mathrm{~g} / \mathrm{L}$ yeast extract and $5 \mathrm{~g} / \mathrm{L} \mathrm{NaCl})[60,61]$ was added and incubated at $37^{\circ} \mathrm{C}$ for $1 \mathrm{~h}$ and $650 \mathrm{rpm}$. Positive clones were streaked out equally onto LB-agar plates supplemented with $100 \mu \mathrm{g} / \mathrm{ml}$ carbenicillin as selection marker.

\subsubsection{Preparation of Plasmid DNA from E.coli Cells}

For isolation of the target plasmid $5 \mathrm{ml} \mathrm{LB}$ medium was incubated with E.coli cells containing the desired plasmid and incubated shaking over night at $30{ }^{\circ} \mathrm{C}$ and $200 \mathrm{rpm}$. Plasmid preparation was performed according to the manufacturer's manual of the NucleoSpin ${ }^{\circledR}$ Plasmid kit and obtained plasmids were stored at $-80{ }^{\circ} \mathrm{C}$.

\subsubsection{DNA Sequencing}

Plasmid DNA was sequenced by a company (Seqlab, Göttingen, Germany) to verify the correctness of the generated construct. For this purpose, 20 pmol of the sequencing primer with $500 \mathrm{ng}$ vector DNA were sent to the company according to their sample shipment requirements.

\subsubsection{Protein Chemistry}

\subsubsection{Overexpression of the GST-hQC Fusion Protein in E.coli BL21 Star Cells}

For expression of the fusion protein one single colony was used to inoculate a $30 \mathrm{ml}$ LB-media culture (10 g/L tryptone, $5 \mathrm{~g} / \mathrm{L}$ yeast extract and $5 \mathrm{~g} / \mathrm{L} \mathrm{NaCl})$ [60] with 100 $\mu \mathrm{g} / \mathrm{ml}$ carbenicillin as selection marker and incubated at $37^{\circ} \mathrm{C}$ and $200 \mathrm{rpm}$ overnight in an incubation shaker. The overnight culture was in turn used to inoculate a $200 \mathrm{ml}$ LB-media culture, again supplemented with $100 \mu \mathrm{g} / \mathrm{ml}$ carbenicillin as selection marker, to an $\mathrm{OD}_{600}$ of 0.1 and incubated at $37^{\circ} \mathrm{C}$ and $200 \mathrm{rpm}$ for $5 \mathrm{~h}$. The $500 \mathrm{ml} \mathrm{LB}$-media 
containing main cultures were supplemented each with $100 \mu \mathrm{g} / \mathrm{ml}$ carbenicillin and $10 \mu \mathrm{M}$ $\mathrm{ZnSO}_{4}$. The trace element was added to ensure that the active site of hQC is fully occupied and prepared according to a standard protocol [62]. Main cultures were inoculated to an $\mathrm{OD}_{600}$ of 0.1 , followed by an incubation step at $37^{\circ} \mathrm{C}$ and $200 \mathrm{rpm}$ in an incubation shaker until an $\mathrm{OD}_{600}$ of 0.6 was reached. Subsequently, cultures were rapidly cooled down to $16{ }^{\circ} \mathrm{C}$ until an $\mathrm{OD}_{600}$ of 0.8 was reached. Gene expression was induced by the addition of $100 \mu \mathrm{M}$ IPTG followed by an incubation step at $16{ }^{\circ} \mathrm{C}$ and $200 \mathrm{rpm}$ for $\sim 65 \mathrm{~h}$. After the incubation period had elapsed, cells were harvested by a centrifugation step at 4800 rpm for 30 min at $4{ }^{\circ} \mathrm{C}$. The harvested cells were either directly used or stored at $-80{ }^{\circ} \mathrm{C}$ until usage.

\subsubsection{Purification of the GST-hQC Fusion Protein}

For a standard purification all steps were performed at $8{ }^{\circ} \mathrm{C}$. First, a $60-80 \mathrm{~g}$ cell pellet was resuspended in $3 \mathrm{ml}$ lysis buffer $\left(300 \mathrm{mM} \mathrm{NaCl}, 50 \mathrm{mM} \mathrm{NaH} \mathrm{PO}_{4}, 1 \mathrm{mM}\right.$ DTT, $5 \mu \mathrm{g} / \mu \mathrm{l}$ DNaseI, spatula tip lysozyme, $\mathrm{pH} 7.5)$ per gram cell pellet, followed by an incubation step for $10 \mathrm{~min}$ under constant stirring. Finally, cells were disrupted by three passages through a microfluidizer. After removal of cell debris by a centrifugation step at $17.500 \mathrm{rpm}$ for $30 \mathrm{~min}$ at $4{ }^{\circ} \mathrm{C}$, obtained supernatant was loaded onto a Glutathione Sepharose ${ }^{\circledR} 4 \mathrm{FF}(16 / 10)$ column equilibrated with loading buffer $(300 \mathrm{mM} \mathrm{NaCl}, 50 \mathrm{mM}$ $\mathrm{NaH}_{2} \mathrm{PO}_{4}, 1 \mathrm{mM}$ DTT, pH 7.5). After a washing step with $100 \mathrm{ml}$ ATP washing buffer (300 $\mathrm{mM} \mathrm{NaCl}, 50 \mathrm{mM} \mathrm{NaH}_{2} \mathrm{PO}_{4}, 1 \mathrm{mM}$ DTT, $5 \mathrm{mM}$ ATP, $5 \mathrm{mM} \mathrm{MgSO}_{4}$, pH 7.5) the fusion protein was eluted with an elution buffer $\left(300 \mathrm{mM} \mathrm{NaCl}, 50 \mathrm{mM} \mathrm{NaH} \mathrm{PO}_{4}, 20\right.$ mM L-Glutathione reduced) and fractions containing the fusion protein were pooled. To prepare cleavage of GST-tag from hQC, fusion protein was dialyzed against two liter dialysis buffer $\left(300 \mathrm{mM} \mathrm{NaCl}, 50 \mathrm{mM} \mathrm{NaH}{ }_{2} \mathrm{PO}_{4}, \mathrm{pH} 7.5\right)$ under constant stirring to remove the reduced L-Glutathione. For the actual cleavage, PreScission Protease was added in a 1:200 ratio (PreScission : fusion protein) to the fusion protein and incubated under gentle shaking for four hours. Afterwards, protein solution was filtered with a $0.2 \mu \mathrm{m}$ filter and prepared for the next purification step. 


\subsubsection{Purification of Untagged hQC}

Again, all purification steps were performed at $8{ }^{\circ} \mathrm{C}$. To remove the GST-tag, the protein solution was loaded a second time onto a Glutathione Sepharose ${ }^{\circledR} 4$ FF (16/10) column equilibrated with dialysis buffer. The flow-through fractions containing hQC were pooled and dialyzed against two liter low salt buffer $\left(50 \mathrm{mM} \mathrm{NaH}_{2} \mathrm{PO}_{4}, \mathrm{pH} 7.5\right.$, conductivity $5.63 \mu \mathrm{S} / \mathrm{cm})$ to remove chloride ions. Afterwards, the protein solution was loaded onto an anion exchange Source 30Q (16/10) column, which was already equilibrated with low salt buffer. When the protein solution was completely loaded and the UV-signals (254 $\mathrm{nm}$ and $280 \mathrm{~nm}$ ) showed a stable signal, a linear gradient (300 ml, $0-100 \%$ B) with high salt buffer ( $1 \mathrm{M} \mathrm{NaCl}, 50 \mathrm{mM} \mathrm{NaH} \mathrm{PO}_{4}, \mathrm{pH} 7.5$, conductivity $41.24 \mu \mathrm{S} / \mathrm{cm}$ ) was started. Fractions containing pure hQC were pooled and concentrated with a Spin-X ${ }^{\circledR}$ UF concentrator (30,000 MWCO) to the desired volume and molarity. Afterwards, prepared hQC was analyzed via SDS-PAGE to check the purity grade. Finally, the enzyme was dialyzed against one liter storage buffer (50 mM Tris/HCl, $\mathrm{pH} 8.0)$ and stored on ice for 1-2 months without losing activity.

\subsubsection{Sodium Dodecyl Sulfate Polyacrylamide Gel Electrophoresis (SDS-PAGE)}

For analysis of purified hQC SDS-PAGE was performed relying on a protocol of Laemmli and Weber $[63,64]$. SDS gels with a $1 \mathrm{~mm}$ thickness contained a stacking part $(5 \%$ acrylamide, $1 \%$ SDS, $125 \mathrm{mM}$ Tris, $\mathrm{pH}$ 6.8) and a separation part (12\% acrylamide, 1 \% SDS, $375 \mathrm{mM}$ Tris, $\mathrm{pH} 8.8$ ). Both parts were step-wise polymerized by adding $0.3 \%$ APS and $0.3 \%$ TEMED. Before the protein samples were loaded onto the stacking gel, they were mixed with SDS sample buffer $(25 \%(\mathrm{w} / \mathrm{v})$ glycerol, $2 \%(\mathrm{w} / \mathrm{v})$ SDS, $0.02 \%$ $(\mathrm{w} / \mathrm{v})$ Bromophenol blue, $100 \mathrm{mM}$ Tris, $\mathrm{pH}$ 6.8) in a $1: 1$ ratio and incubated at $96{ }^{\circ} \mathrm{C}$ for five minutes. Gels ran with $1 \mathrm{~mA} / \mathrm{cm}^{2}$ in SDS running buffer (200 mM glycine, 25 $\mathrm{mM}$ Tris, $3.5 \mathrm{mM}$ SDS, $\mathrm{pH}$ 8.3) until the front of the loading dye reached the bottom of the gel. Next, the gels were stained with Coomassie Brilliant Blue $(0.25 \%$ Coomassie Brilliant Blue, $30 \%$ ethanol, $6 \%$ acetic acid) for $10 \mathrm{~min}$ and afterwards decolorized with a destaining solution (30\% ethanol, $10 \%$ acetic acid) for additional $10 \mathrm{~min}$. 


\subsubsection{Preparation of Cobalt(II)-Substituted hQC}

\subsection{Generation of Apo-hQC}

For stopped-flow experiments, a modified protocol provided by Probiodrug [23] was used for substitution of zinc against spectroscopically active cobalt within the catalytic site of hQC. To generate apoenzyme, hQC was stepwise dialyzed at $8{ }^{\circ} \mathrm{C}$ against 250,250 and $500 \mathrm{ml}$ chelating buffer $\left(300 \mathrm{mM} \mathrm{NaCl}, 50 \mathrm{mM} \mathrm{NaH} \mathrm{PO}_{4}, 5 \mathrm{mM}\right.$ EDTA, $5 \mathrm{mM} \mathrm{1,10-}$ phenanthroline, $\mathrm{pH} 7.5$ ) for 4,4 and $16 \mathrm{~h}$, respectively. Next, to remove all chelating reagents, the apoenzyme was dialyzed against a total volume of three liters metal-free buffer $\left(50 \mathrm{mM}\right.$ Tris $/ \mathrm{HCl}, 25 \mathrm{~g} / \mathrm{l}$ Chelex-100, $\mathrm{pH} 8.0$ ) at $8{ }^{\circ} \mathrm{C}$. To ensure removal of the active site zinc ion, the apoenzyme was tested for activity (see 5.2.3.1) and should show $<1 \%$ residual activity compared to the holoenzyme. In addition, absorption at $260 \mathrm{~nm}$ was checked to guarantee complete elimination of 1,10-phenanthroline. After removal of the zinc ion and generation of the apoenzyme all used commodities, storage conditions of solutions as well as further working steps were performed under metal-free conditions.

\subsection{Reconstitution of Cobalt(II) within the Active Site}

The apoenzyme was desalted against substitution buffer $\left(50 \mathrm{mM} \mathrm{NaH}{ }_{2} \mathrm{PO}_{4}, 100 \mathrm{mM}\right.$ $\mathrm{NaCl}$; pH 6.8) with a HiPrep ${ }^{T M} 26 / 10$ Desalting column and afterwards diluted to a final concentration of $40 \mu \mathrm{M}$. In order to reconstitute the active site of hQC with cobalt the apoenzyme was incubated at room temperature for 15 min with a 6 -folded excess of $\mathrm{CoCl}_{2}$ (f.c $240 \mu \mathrm{M}$ ). After incorporation of cobalt within the active site of hQC the solution showed an optically change from colorless into light pink. Next, Co(II)-hQC was desalted against storage buffer $(50 \mathrm{mM}$ Tris/ $\mathrm{HCl}, \mathrm{pH} 8.0)$ and the specific activity was measured to check if $\mathrm{Co}(\mathrm{II})-\mathrm{hQC}$ has comparable activity compared to $\mathrm{Zn}$ (II)-hQC (see $5.2 .3 .1)$.

\subsubsection{Determination of Protein Concentration}

Concentration of hQC was measured spectroscopically at $280 \mathrm{~nm}$ with a NanoDrop2000. In order to calculate the protein concentration of hQC, the experimentally determined mo- 
lar extinction coefficient $\left(\varepsilon_{\mathrm{hQC}}=55869 \mathrm{M}^{-1} \mathrm{~cm}^{-1}\right)$ and the molecular weight $\left(\mathrm{MW}_{\mathrm{hQC}}\right.$ $=37511 \mathrm{~g} / \mathrm{mol}$, ProtParam) were used. The molar extinction coefficient was determined according to an established protocol [65].

\subsubsection{Kinetic Methods}

\subsubsection{Coupled Spectroscopic Steady-State Assay}

Enzymatic activity of hQC for conversion of substrates harboring an N-terminal Gln into pGlu was monitored spectrophotometrically in a coupled steady state assay using the auxiliary enzyme GLDH [66]. The assay is based on the coupling of ammonia, which is formed and released after cyclization of N-terminal glutamine residues, to the GLDH. It is important that detection of ammonia by this assay is proportional to the decrease of absorbance at $\lambda=340 \mathrm{~nm}$ over time. Here, NADH gets oxidized to $\mathrm{NAD}^{+}$, which shows no absorbance at this range (Fig. 5.1A). The coupled steady-state assay is shown in Fig. 5.1B schematically.

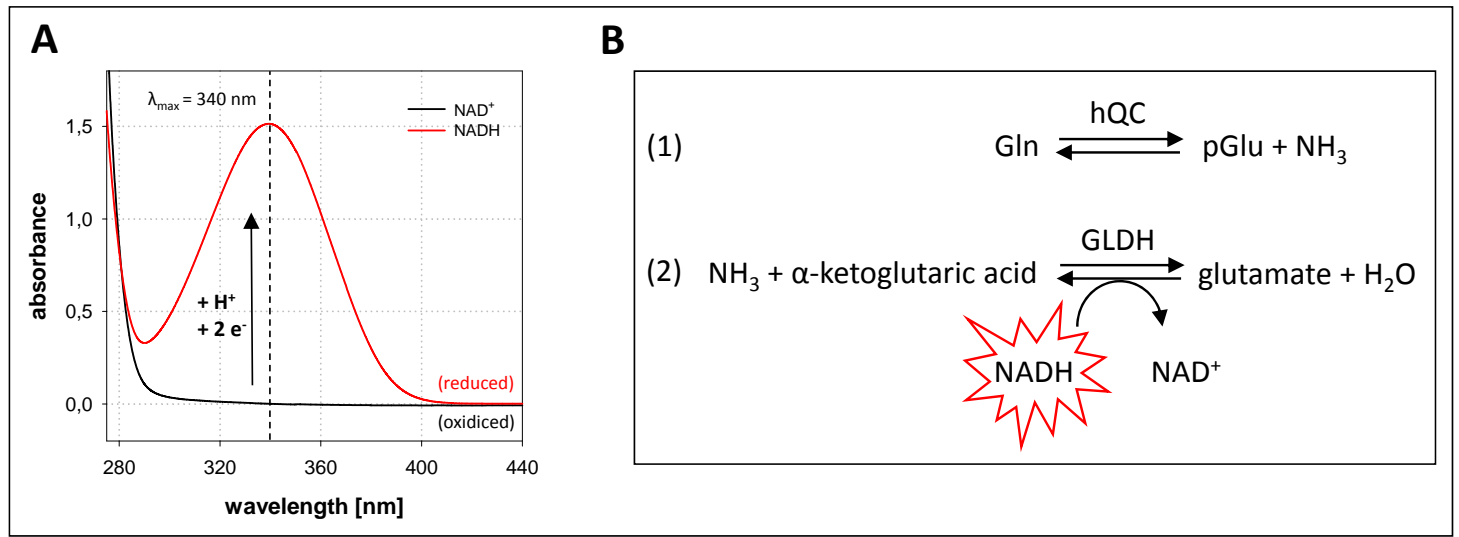

Figure 5.1: General overview of the spectrophotometric coupled steady-state assay. (A) $\mathrm{UV} / \mathrm{V}$ is spectra of $\mathrm{NAD}^{+} / \mathrm{NADH}$. NADH has an absorption maximum at $\lambda=340 \mathrm{~nm}$, whereas $\mathrm{NAD}^{+}$ does not absorb in this range. (B) Schematic representation of the coupled steady-state assay. Catalysis of N-terminal glutamine to pyroglutamic acid (pGlu) by hQC is linked to the formation of glutamate (Glu) using the auxiliary enzyme glutamate dehydrogenase (GLDH).

A typical standard reaction with a total volume of $500 \mu \mathrm{l}$ at saturated conditions consists of $50 \mathrm{mM}$ Tris/HCl, pH 8.0, buffer, $0.3 \mathrm{mM} \mathrm{NADH}\left(K_{\mathrm{M}}=0.024 \mathrm{mM}[66]\right), 15 \mathrm{mM} \alpha$ ketoglutaric acid $\left(K_{\mathrm{M}}=0.7 \mathrm{mM}[66]\right), 2.5 \mathrm{mM}$ Gln-substrate and $30 \mathrm{U} / \mathrm{ml}$ GLDH. The reaction was started by adding $1 \mu \mathrm{g}$ of hQC and measured at $30{ }^{\circ} \mathrm{C}$. To calculate the specific activity $\left(A_{\mathrm{spec}}\right)$, change in absorbance per minute $(\Delta E / \mathrm{min})$, total volume of the 
reaction $\left(V_{\text {total }}\right)$, path length of the cuvette $(d)$, molar extinction coefficient of NADH $\left(\varepsilon_{\mathrm{NADH}}=6200 \mathrm{M}^{-1} \mathrm{~cm}^{-1}\right.$ at $\left.340 \mathrm{~nm}\right)$, volume of hQC solution added into the cuvette $\left(V_{\mathrm{E}}\right)$ and hQC concentration of the stock solution $\left(c_{\mathrm{stock}}\right)$ were used according to Eq. 2

$$
A_{\text {spec }}=\frac{\Delta E / \mathrm{min} \cdot V_{\mathrm{total}} \cdot 1000}{d \cdot \varepsilon_{\mathrm{NADH}} \cdot V_{\mathrm{E}} \cdot c_{\mathrm{stock}}}
$$

Dependency of the initial rates on the substrate concentration were analyzed according to Eq. 3, where $V_{\max }$ is the maximum rate, $K_{\mathrm{M}}$ the Michaelis-Menten constant and $[S]$ the concentration of the substrate.

$$
v=\frac{V_{\max } \cdot[S]}{K_{\mathrm{M}} \cdot[S]}
$$

Data analysis and curve fitting was done with the SigmaPlot software.

\subsubsection{Continuous Calorimetric Activity Assay for Human Glutaminyl Cyclase using Isothermal Titration Calorimetry}

An alternative kinetic assay, using Isothermal titration calorimetry (ITC), which is rapid, simple and precise, was facilitated for this study. It has been already known that calorimetry can be used to measure released or consumed heat during enzymatic activity [67] and that the observed thermal power is directly proportional to the rate of an enzymatic reaction [68].

Conversion of substrate to product by a catalyst generates or releases heat, which corresponds to the molar reaction enthalpy $\Delta \mathrm{H}$ of the reaction. This $\Delta \mathrm{H}$ for a defined substrate amount can be directly obtained from the total heat of an ITC experiment at the time point when substrate or enzyme is injected and the reaction starts and proceeds further until the substrate is completely converted into product. The change in thermal power while substrate is depleted over time can be continuously monitored and therefore heat (Q) is measured as a function of time and defined as the differential thermal power (dp).

$$
\text { Power }=d p=\frac{d Q(t)}{d t}
$$


To obtain the experimental $\Delta \mathrm{H}$-value, it is necessary to integrate the different dp-values and divide the sum of all integrals by the total substrate amount $n(S)_{\text {total }}$. For simplification $\mathrm{n}(\mathrm{S})_{\text {total }}$ is defined as the initial substrate concentration at time point zero $[\mathrm{S}]_{0}$ and the total Volume $\mathrm{V}$ of the measurement cell.

$$
\Delta H=\frac{1}{[S]_{0} \cdot V} \int_{0}^{\infty} \frac{d Q(t)}{d t} d t
$$

To ensure accurate overall integral calculation of the reaction, it is necessary to generate a high number of small integrals. Thereby, every generated data point $\left(\mathrm{dp}_{t}\right)$, which displays a dp-value at a defined time point, has to be multiplied with the spacing (s) of each generated data point t to approximate each integral.

$$
\int d p \approx \sum_{t=0}^{t_{i}} d p_{t} \cdot s
$$

Every square formed integral is defined by the different spacing- and dp-values, thereby generating an approximated overall surface integral, which describes the catalyzed conversion of substrate $(\mathrm{S})$ to product $(\mathrm{P})$. The introduced/dissipated amount of energy is described by the surface area of each integral and needs to be translated into a defined amount of substrate into product for each interval $t\left(n_{t}(P)\right.$ by dividing the area against the experimentally determined $\Delta \mathrm{H}$-value.

$$
n_{t}(P)=\frac{d p_{t} \cdot s}{\Delta H}
$$

It is necessary to accumulate product formation from $t_{0}-t$ to determine total product formation at a defined time point $\left.t\left(n_{t, t o t a l}\right)\right)$.

$$
n_{t, \text { total }}(P)=\sum_{t=0}^{t} n_{t}(P)
$$

Due to the fact that the initial substrate amount $n_{0}(S)$ is known and every substrate molecule is irreversibly converted into one product molecule, it is possible to calculate the amount of substrate molecules $n_{t, t o t a l}(S)$ at each time point $t$ by using the following 
equation:

$$
n_{t, \text { total }}(S)=n_{0}(S)-\sum_{t=0}^{t} n_{t}(P)
$$

To finally display the substrate concentration at every time point $\left(c_{t}(S)\right)$ on the $\mathrm{x}$-axis for the Michaelis-Menten plot, it is necessary to use the previously calculated amount of substrate molecules $n_{t, \text { total }}(S)$ at each time point and divide it by the volume of the measurement cell $(V)$ of the ITC device.

$$
c_{t}(S)=\frac{n_{t, t o t a l}(S)}{V}
$$

Next, to calculate the reaction velocity at a given time point $\left(v_{t}\right)$ every corresponding dp-value needs to be divided by the experimentally determined $\Delta \mathrm{H}$-value.

$$
v_{t}=\frac{d p_{t}}{\Delta H}
$$

At last, to define the $\mathrm{y}$-axis the reaction velocity at a given time point $\left(v_{t}\right)$ needs to be converted into specific activity $\left(A_{\text {spec }}\right)$ or into the turnover number $\left(k_{\text {cat }}\right)$ by using the following equations:

$$
\begin{gathered}
A_{\text {spec }}=\frac{v_{t}}{n(h Q C)} \\
k_{\text {cat }}=\frac{v_{t} \cdot 60}{M W(h Q C)}
\end{gathered}
$$

Now, the specific activity or turnover number can be plotted against the substrate concentration $c_{t}(S)$ and is fitted according to the Michalis-Menten equation (see eq. 3), if the reaction follows the kinetics of the Michaelis-Menten model.

For a conventional reaction all solutions were degassed and filtered. In addition, the samples were centrifuged at $15.000 \mathrm{rpm}$ for $10 \mathrm{~min}$ at $4{ }^{\circ} \mathrm{C}$. The reference cell of the ITC device was filled with water. Sample concentrations and ITC setup were utilized as described in Tab. 5.2 and Tab. 5.2. Data analysis and curve fitting was performed with the Excel and SigmaPlot softwares. 
Table 5.2: ITC setup for single injection experiments.

\begin{tabular}{l|c|c} 
& Gln-substrates & Glu-substrates \\
\hline Number of injections & 2 & 2 \\
Sample cell temperature $\left[{ }^{\circ} \mathrm{C}\right]$ & 30 & 30 \\
Ref. power $[\mu \mathrm{cal} / \mathrm{s}]$ & 5 & 5 \\
Initial delay $[\mathrm{s}]$ & 60 & 120 \\
Stirring speed $[\mathrm{rpm}]$ & 500 & 500 \\
Spacing injection $1[\mathrm{~s}]$ & 3600 & 20000 \\
Spacing injection 2 $[\mathrm{s}]$ & 120 & 300 \\
Injection volume $[\mu \mathrm{l}]$ & 10 & 10 \\
Filter period $[\mathrm{s}]$ & 5 & 5
\end{tabular}

Table 5.3: Used concentrations for the continuous calorimetric activity assay. Volumes of the sample cell and the injection syringe are $204,5 \mu \mathrm{l}$ and $40 \mu \mathrm{l}$, respectively.

\begin{tabular}{c|c|c||c|c|c} 
Sample cell & concentration & $\mathrm{pH}$ & Injec. syringe & concentration & $\mathrm{pH}$ \\
\hline QQ & $2 \mathrm{mM}$ & $8.0^{A}$ & $\mathrm{hQC}$ & $1 \mu \mathrm{M}$ & $8.0^{A}$ \\
QFRH & $2 \mathrm{mM}$ & $8.0^{A}$ & $\mathrm{hQC}$ & $1 \mu \mathrm{M}$ & $8.0^{A}$ \\
EFRH & $3 \mathrm{mM}$ & $6.8^{B}$ & $\mathrm{hQC}$ & $60 \mu \mathrm{M}$ & $6.8^{B}$ \\
QLYEN & $2 \mathrm{mM}$ & $8.0^{A}$ & $\mathrm{hQC}$ & $1 \mu \mathrm{M}$ & $8.0^{A}$ \\
QHWSY & $2.5 \mathrm{mM}$ & $8.0^{A}$ & $\mathrm{hQC}$ & $1 \mu \mathrm{M}$ & $8.0^{A}$ \\
QGPWL & $1 \mathrm{mM}$ & $8.0^{A}$ & $\mathrm{hQC}$ & $10 \mu \mathrm{M}$ & $8.0^{A}$ \\
QPLPD & $3 \mathrm{mM}$ & $8.0^{A}$ & $\mathrm{hQC}$ & $10 \mu \mathrm{M}$ & $8.0^{A}$
\end{tabular}

${ }^{A}$ solutions were buffered in $50 \mathrm{mM}$ Tris/HCl.

$B$ solutions were buffered in $30 \mathrm{mM}$ Tris/HCl and $20 \mathrm{mM}$ MES

\subsubsection{1 pH-Dependency of Human Glutaminyl Cyclase at Constant lonic Strength}

Reactions were prepared as described previously (see chapter 5.2.3.2). To enable a wide buffer capacity with constant ionic strength from pH 5.5 to 9.5, the normal measuring buffer was replaced by a three component buffer consisting of $0.05 \mathrm{M}$ acetic acid, 0.05 M MES, 0.1 M Tris [69]. Data points were fitted according to the following equation, in which $v$ stands for the reaction velocity, $\mathrm{pH}$ for the negative logarithm of the molarity of 
the $\mathrm{H}^{+}$ions and $\mathrm{p} K_{a}$ for the negative logarithm of the acid dissociation constant $K_{\mathrm{a}}$.

$$
v=v_{\max } \cdot \frac{1}{1+10^{\left(p K_{a 1}-p H\right)}+10^{\left(p K_{a 2}-p H\right)}}+v_{\min }
$$

\subsection{Kinetic Characterization of Human Glutaminyl Cyclase Inhibitors}

For characterization of different inhibitors for hQC a standard reaction was prepared as described previously (see chapter 5.2.3.2). As a variation, the substrate with a defined concentration of the inhibitor was loaded together into the measurement cell. To determine the inhibition constant $K_{\mathrm{i}}$, obtained data points of the Michaelis-Menten plot were analyzed accordingly to Dixon [70]. The Dixon plot represents the reciprocal initial reaction velocity $v$, which is plotted against the inhibitor concentration [I] at various fixed concentrations of substrate [S]. Intersections of obtained linear regressions were calculated to obtain the inhibition constant $K_{\mathrm{i}}$. In addition, experimental data was plotted three-dimensionally and obtained data points were fitted according to Eq. 15 [71].

$$
v=\frac{V_{\max } \cdot[S]}{K_{M} \cdot\left(1+\frac{[I]}{K_{i}}\right)+[S]}
$$

\subsubsection{Fast Kinetics Stopped-Flow UV/Vis Spectroscopy}

To gather information about the overall enzymatic reaction and the coordination number via the electronic environment of the substituted cobalt ion within the active site of hQC, an Applied Photophysics SX18 MV stopped-flow spectrophotometer with a photo diode array was used. For this purpose, different substrates were rapidly mixed with the enzyme with a dead response time of 1.5 milliseconds. Enzyme and substrates were mixed in a one to one volume ratio within the $20 \mu \mathrm{l}$ measurement cell of the stopped-flow device with an optical path length of $10 \mathrm{~mm}$. Time-resolved spectra were collected in equidistant time intervals in a wavelength range from $185-723 \mathrm{~nm}$. Experiments were performed at $10{ }^{\circ} \mathrm{C}$ for glutaminyl substrates and for glutamyl substrates at $30{ }^{\circ} \mathrm{C}$. For each data collection the first $0.1 \mathrm{~s}$ was measured with 10 data points and the following $150 \mathrm{~s}$ with 200 data points. Final concentrations of enzyme and substrates after mixing are shown individually 
in Tab. 5.4.

Before usage, syringes and the measurement cell of the stopped-flow device were incubated in a solution containing $2 \%$ Hellmanex and $5 \mathrm{mM}$ EDTA for 20 min to remove free metal ions. After washing the system sufficiently with water a spectrum of the acquired buffer was measured. Subsequently, a spectrum of the cobalt substituted enzyme was recorded before it was rapidly mixed with the substrate. Obtained spectra were buffer- and proteincorrected by utilizing the softwares SX-Prodata Viewer and SigmaPlot 11.

Table 5.4: Final concentration of compounds after mixing in the measurement cell.

\begin{tabular}{|c|c|}
\hline Compound & Final concentration $[\mathrm{mM}]$ \\
\hline $\mathrm{Co}(\mathrm{II})-\mathrm{hQC} \mathrm{C}_{35-361}$ & $0.1^{A, B}$ \\
\hline QQ & $8^{A}$ \\
\hline QFRH & $8^{A}$ \\
\hline EFRH & $1^{B}$ \\
\hline Neurotensin $_{1-5}$ & $8^{A}$ \\
\hline Gastrin $_{1-5}$ & $8^{A}$ \\
\hline $\mathrm{GnRH}_{1-5}$ & $8^{A}$ \\
\hline Orexin $\mathrm{A}_{1-5}$ & $8^{A}$ \\
\hline
\end{tabular}

\subsubsection{Biophysical Methods}

\subsubsection{UV/Vis Absorption Spectroscopy}

All UV/Vis spectra were measured between 300 - $700 \mathrm{~nm}$ with an acquisition speed of 100 $\mathrm{nm} / \mathrm{min}$ in a Jasco V-650 spectrophotometer. A quartz cuvette with a path length of 1 $\mathrm{cm}$ and a band width of $1 \mathrm{~nm}$ was used for all measurements. Furthermore, measurements were performed at $25{ }^{\circ} \mathrm{C}$ with a data pitch of $1 \mathrm{~nm}$. All utilized solutions were treated with Chelex-100 before usage to guarantee metal-free conditions. Difference spectra were generated by subtracting free cobalt and protein spectra from the recorded one. 


\subsection{Titration of Cobalt to Apoenzyme}

Ten titration steps, each containing one microliter of $14-24 \mathrm{mM} \mathrm{CoCl}_{2}$, were titrated into a total volume of $1 \mathrm{ml} 40 \mu \mathrm{M}$ apoenzyme. Afterwards, samples were incubated for $4 \mathrm{~min}$ and subsequently, spectra were measured in the spectrophotometer. Titration experiments were performed at alkaline (50 mM Tris/HCl, $\mathrm{pH} 8.0)$ and weak acidic $\left(50 \mathrm{mM} \mathrm{NaH}_{2} \mathrm{PO}_{4}\right.$, $300 \mathrm{mM} \mathrm{NaCl}, \mathrm{pH} 6.8$ ) conditions. To quantify cobalt incorporation, absorption at 550 $\mathrm{nm}\left(A_{\max }\right)$ of the corrected spectrum was plotted against the cobalt concentration. In order to determine the dissociation constant $\left(K_{\mathrm{d}}\right)$ data was fitted according to:

$$
A=\frac{A_{\max } \cdot[C o]}{K_{\mathrm{d}} \cdot[C o]}
$$

\subsection{Stability of Cobalt Substituted Human Glutaminyl Cyclase}

To see if the cobalt ion forms a stable complex within the active site hQC, $40 \mu \mathrm{M}$ apoenzyme were supplemented with $144 \mu \mathrm{M} \mathrm{CoCl}_{2}$ and incubated for defined time points until spectrum was measured.

\subsubsection{Quantitative Characterization of the Thermodynamics of Binding Events using Isothermal Titration Calorimetry}

To obtain thermodynamic parameters of interactions in solution, the binding event of small ligands to glutaminyl cyclase were studied utilizing Isothermal Titration Calorimetry. In this study ITC was used to investigate the interaction between hQC and different potential inhibitors. Instead of measuring stepwise changes in the enthalpy of interaction during the course of multiple titration steps only a single titration step was carried out to obtain the overall enthalpy of the binding process.

Before measurement, the enzyme was dialyzed against one liter $50 \mathrm{mM}$ Tris/HCl, pH 8.0, for 12 hours at $8^{\circ} \mathrm{C}$. In addition, the ligand was dissolved in the identical dialysis buffer. Concentrations and ITC setup are listed in Tab. 5.5. Data analysis was performed with MicroCal Analysis and SigmaPlot software. 
Because the overall binding enthalpy and the association constant are determined experimentally the remaining thermodynamic parameters were calculated according to Eq. 17.

$$
\Delta G=-R T \cdot \ln \left(K_{a}\right)=\Delta H-T \Delta S
$$

$\Delta G=$ Gibbs energy changes

$\Delta H=$ Enthalpy changes

$\Delta S \quad=$ Entropy changes

$T=$ Absolute temperature in Kelvin

$K_{a}=$ Association constant $\left[\mathrm{M}^{-1}\right]$

$R \quad=$ Ideal gas constant $\left(8.314 \cdot 10^{-3} \mathrm{~kJ} \mathrm{~mol}^{-1} \mathrm{~K}^{-1}\right)$

Table 5.5: Used ITC setup and protein-ligand concentrations for thermodynamic characterization.

\begin{tabular}{l|l} 
Number of injections & 3 \\
Injection syrringe & $25 \mathrm{mM} \mathrm{Q}$ (hydrazide)-FA \\
Sample cell & $0.08 \mathrm{mM}$ hQC \\
Sample cell temperature $\left[{ }^{\circ} \mathrm{C}\right]$ & 30 \\
Ref. power $[\mu \mathrm{cal} / \mathrm{s}]$ & 10 \\
Stirring speed $[\mathrm{rpm}]$ & 500 \\
Spacing after injection $[\mathrm{s}]$ & 300 \\
Injection volume $[\mu \mathrm{l}]$ & 5 \\
Filter period $[\mathrm{s}]$ & 2
\end{tabular}

\subsubsection{Conformational Stability of Human Glutaminyl Cyclase in Dependence of pH or Temperature using Circular Dichroism Spectroscopy}

Numerous intra-molecular interactions of proteins, e.g. formation of disulfide bridges between cysteines or hydrogen bonds among different amino acid residues, ensure conformational stability and define secondary structure elements. If a protein molecule undergoes an unfolding transition due to breakup of these intra-molecular interactions, it loses its secondary structure elements. This transition can be monitored via a Chirascan CD-spectropolarimeter. The CD-signal arises if the absorption of the left- and the right-circular polarized light of an asymmetric optical center are unequal and and gets 
diminished due to protein unfolding. To quantify secondary structure elements in dependence of $\mathrm{pH}$-value, $\mathrm{CD}$-spectra were recorded for $\mathrm{hQC}$ in the far $\mathrm{UV} / \mathrm{V}$ is range. In addition, thermal stability of hQC was analyzed by inducing a continuous temperature transition from $20^{\circ} \mathrm{C}$ to $96^{\circ} \mathrm{C}$.

In case for $\mathrm{pH}$-stability experiments of hQC, spectra were recorded in one nanometer steps in the far UV/Vis range from 190-280 nm with a path length of one millimeter at $30^{\circ} \mathrm{C}$. For measurements $0.1 \mathrm{mg} / \mathrm{ml} \mathrm{hQC}$ was diltued into a $10 \mathrm{mM}$ potassium phosphate buffer with varing $\mathrm{pH}$-values from 6.16-8.45.

For thermal stability experiments $0.1 \mathrm{mg} / \mathrm{ml} \mathrm{Zn(II)-hQC,} \mathrm{Co(II)-hQC} \mathrm{or} \mathrm{apoenzyme} \mathrm{di-}$ luted in $10 \mathrm{mM}$ Tris/HCl, $\mathrm{pH}$ 8.0, were characterized. Settings of a typical measurement are listed in Tab. 5.6. Additionally, to compare the native and denaturated enzyme, CDspectra were taken before and after temperature transition. The midpoint of the thermal transition was determined according to Eq. 18 [72].

$$
\begin{array}{ll}
y=\frac{\left(y_{f}+m_{f} \cdot T\right)+\left(y_{u}+m_{u} \cdot T\right) \cdot \exp \left[\left(\frac{\Delta H_{m}}{R T}\right) \cdot\left(\frac{T-T_{m}}{T_{m}}\right)\right]}{1+\exp \left[\left(\frac{\Delta H_{m}}{R T}\right) \cdot\left(\frac{T-T_{m}}{T_{m}}\right)\right]} \\
\quad=\text { CD-signal at } 196 \mathrm{~nm} \\
y \quad=\text { Intercepts of pre- and posttransition baselines } \\
y_{f}, y_{u} \quad=\text { Slopes of pre- and posttransition baselines } \\
m_{f}, m_{u} \quad & \text { Temperature in Kelvin } \\
T & =\text { Midpoint of the thermal unfolding curve } \\
T m & =\text { Enthalpy change for unfolding at Tm } \\
\Delta H_{m} & =\text { Ideal gas constant } \\
R &
\end{array}
$$

This equation holds true for determination of the midpoint for reversibly unfolding proteins. However, for irreversibly unfolding proteins the thermal transition depends on the heating rate and exposure time at a certain temperature, which is the case for glutaminyl cyclase. As a consequence, the determined thermal transition midpoints are an apparent 
value. Nevertheless, the midpoints are adequate measures for a comparative examination.

\begin{tabular}{l|l}
$\begin{array}{l}\text { Table 5.6: Settings of the CD- } \\
\text { spectrometer for the thermal denat- } \\
\text { uration. }\end{array}$ \\
$\begin{array}{l}\text { Temperature start } \\
\text { Temperature end }\end{array}$ & $20^{\circ} \mathrm{C}$ \\
$96^{\circ} \mathrm{C}$ \\
Data interval & $0.5{ }^{\circ} \mathrm{C}$ \\
Heating rate & $2.0{ }^{\circ} \mathrm{C} / \mathrm{min}$ \\
Tolerance & $0.2{ }^{\circ} \mathrm{C}$ \\
Wavelength $[\lambda]$ & $196 \mathrm{~nm}$ \\
Time per point & $12 \mathrm{~s}$ \\
Path length & $1 \mathrm{~mm}$
\end{tabular}

Data analysis and curve fitting was done with the SigmaPlot software.

\subsubsection{Oligomerization State of Human Glutaminyl Cyclase Examined via Analytical Ultracentrifugation}

In order to get a quantitative analysis of the oligomerization state of glutaminyl cyclase in solution, analytical ultracentrifugation [73] was used. All experiments in this study were performed by Prof. Dr. Ute Curth from the Institute for Biophysical Chemistry, Hannover Medical School.

\subsubsection{Liquid Chromatography - Mass Spectrometry (LC-MS)}

Target protein was digested with trypsin via in-gel digestion as described in literature $[74,75]$. For protein identification, liquid chromatography was coupled to an Orbitrap Velos Pro mass spectrometer (Thermo Scientific). Chromatographically eluting peptides were online ionized by nano-electrospray (nESI) using the Nanospray Flex Ion Source (Thermo Scientific) at $2.4 \mathrm{kV}$ and continuously transferred into the mass spectrometer. LC-MS method programming and data acquisition was done with the software XCalibur 2.2 (Thermo Scientific). For protein identification MS/MS2 data were searched against a human FASTA-database expanded for the amino acid sequences of interest using the Proteome Discoverer software and the SequestHT search engine. All LC-MS experiments 
were performed by Dr. Oliver Valerius from the Department of Molecular Microbiology and Genetics, Georg-August-University Göttingen.

\subsubsection{X-ray Crystallography}

\subsubsection{Crystallization and Data Collection}

In order to crystallize hQC the hanging drop vapor diffusion method was used. Therefore, common 24-well crystallization plates with sealant were applied and each well was sealed by putting a silanized cover glass on top of the well. Before crystallization the enzyme was rebuffered in crystallization buffer containing $50 \mathrm{mM}$ Tris/ $\mathrm{HCl}$ amd $\mathrm{NaCl}, \mathrm{pH}$ 8.0. The N-terminal shortened version of human glutaminyl cyclase was subjected to initial crystallization trials at a concentration of $10 \mathrm{mg} / \mathrm{ml}$ as described recently [20]. Diffracting crystals grew within one week at $25{ }^{\circ} \mathrm{C}$ in $1.6 \mathrm{M}$ ammonium sulfate, $4 \%$ 1,4-dioxane and 0.1 M MES, pH 6.5, by mixing equal volumes of the enzyme solution and the reservoir. Prior flash cooling with liquid nitrogen, generated crystals were incubated in a cryoprotectant solution containing reservoir solution supplemented with $25 \%$ (v/v) glycerol, 5 $\%(\mathrm{v} / \mathrm{v})$ PEG 400 and $10 \mu \mathrm{M}$ zinc sulfate. In case of substrate soaking experiments the cryoprotectant solution was additionally supplemented with the corresponding substrate and protein crystals were incubated at room temperature for a defined time (Tab. 5.7). Data collection of different crystal structures was performed at different synchrotron facilities and resulting oscillation images were integrated, merged, and scaled using XDS [76, 77]. $\mathrm{Zn}(\mathrm{II})-\mathrm{hQC}_{35-361}$ in absence of substrate crystallized in trigonal space group R32 with unit cell parameters of $\mathrm{a}=\mathrm{b}=119 \AA, \mathrm{c}=333 \AA$ and $\alpha=\beta=90^{\circ}, \gamma=120^{\circ}$ and harbors two molecules per asymmetric unit.

\subsubsection{Structure Determination and Refinement}

Initial phases for each structure were obtained by the molecular replacement method with Phaser [78] using the structure of the already resolved hQC (Protein Data Bank code 2AFM) as search model. Refinement calculations were performed with PHENIX [79] using standard parameters and after each refinement cycle the model was manually checked and rebuilt using Coot [80] until final resolution was reached. Refinement with PHENIX also 
included adding solvent molecules like water automatically. At least, ligands were added into the three dimensional model manually via Coot. Peptide-dependent ligands were build using UCSF Chimera [81], which already included all necessary restrains. As an exception, restraints for the product pEFRH for refinement with PHENIX were generated with the PRODRG server [82]. For the ligand E( $\gamma$-hydrazide)FA the peptide was added via Coot first and after a refinement cycle the hydrazide function was introduced into the side chain of the glutamine by modeling an additional amino group into the corresponding mFo-DFc electron density map. The corresponding bond lengths of the hydrazide function relied on standard bond lengths derived from the Cambridge Structural Database (CSD). Validation of the refined models was carried out using Coot and MOLPROBITY and preparation of figures was performed with PYMOL.

Table 5.7: Substrate soaking conditions and data collection information for all presented crystal structures.

\begin{tabular}{|c|c|c|c|}
\hline Structure & Soaking conditions & Beamline & $\begin{array}{c}\text { Resolution } \\
[\AA]]\end{array}$ \\
\hline $\mathrm{Zn}(\mathrm{II})-\mathrm{hQC}_{35-361}$ & 10 s cryoprotectant & $\begin{array}{l}\text { ESRF } \\
\text { ID23-1 }\end{array}$ & 2.14 \\
\hline $\begin{array}{c}\mathrm{Zn}(\mathrm{II})-\mathrm{hQC}_{35-361} \\
+\mathrm{QFRH}\end{array}$ & $\begin{array}{l}10 \text { s cryoprotectant } \\
+10 \mathrm{mM} \text { QFRH }\end{array}$ & $\begin{array}{c}\text { MAXIV } \\
1911-3\end{array}$ & 1.90 \\
\hline $\begin{array}{l}\mathrm{Zn}(\mathrm{II})-\mathrm{hQC}_{35-361} \\
+\mathrm{EFRH}\end{array}$ & $\begin{array}{c}30 \text { min cryoprotectant } \\
+10 \mathrm{mM} \text { EFRH }\end{array}$ & $\begin{array}{c}\text { MAXIV } \\
1911-3\end{array}$ & 1.92 \\
\hline $\begin{array}{l}\mathrm{Zn}(\mathrm{II})-\mathrm{hQC}_{35-361} \\
\quad+\mathrm{pEFRH}\end{array}$ & $\begin{array}{l}10 \text { min cryoprotectant } \\
+10 \mathrm{mM} \mathrm{pEFRH}\end{array}$ & $\begin{array}{c}\text { MAXIV } \\
1911-3\end{array}$ & 1.88 \\
\hline $\begin{array}{c}\mathrm{Zn}(\mathrm{II})-\mathrm{hQC}_{35-361} \\
+ \\
\mathrm{Q} \text { (hydrazide)-FA }\end{array}$ & $\begin{array}{c}45 \text { min cryoprotectant } \\
+20 \mathrm{mM} \\
\mathrm{Q} \text { (hydrazide)-FA }\end{array}$ & DESY P13 & 1.95 \\
\hline
\end{tabular}




\section{Results and Discussion}

\subsection{Expression and Purification of the $\Delta 34$ Deletion Variant of Human Glutaminyl Cyclase Results in a Correctly Folded Soluble Monomer}

The coding sequence of human pituitary glutaminyl cyclase (QPCT) [9], which is optimized for codon usage in Escherichia coli cells, was used during this study. Chromosomal location of the QPCT gene is at position $2 \mathrm{p} 22.2$ in the human genome and has an open reading frame of 1086 bases, coding for a protein sequence consisting of 361 amino acids with a molecular weight of $40,8 \mathrm{kDa}$. For this study the first $34 \mathrm{~N}$-terminal amino acids were deleted to generate a $\Delta 34$ deletion variant. It was shown that these first $34 \mathrm{~N}$-terminal amino acids encode only for secretion signal, which has no impact on the catalytic activity or on the tertiary protein structure of the enzyme [21]. Furthermore, native human glutaminyl cyclase (hQC) is a glycoprotein (N-glycosylation site at Asn49) harboring a disulfide bond near the catalytic site. Recent studies have shown that this disulfide bond has a stabilizing function and is not essential for catalytic activity [22]. Due to the lack of post-translational modifications, leading to no glycosylation and disulfide bond formation event, solubility problems occur during expression in a prokaryotic host. Protein solubility predictions with the ccSOL omics webserver [83] resulted in low heterologous solubility chances of hQC expressed in Escherichia coli. To overcome the problems of low recovery and heterogeneity of the recombinant $\Delta 34$ deletion variant of hQC the protein-coding sequence was cloned into the pGEX-6P-1 expression vector (Fig. 8.1). The pGEX-6P-1 expression vector encodes for a glutathione S-transferase (GST) affinity tag (MW $=26$ $\mathrm{kDa}$ ), which is located in front of the generated $\Delta 34$ deletion variant of hQC and allows purification of hQC via affinity chromatography [84]. The resulted GST-hQC fusion protein consists of 555 amino acids (Fig. 8.2) with a predicted molecular weight of $63.3 \mathrm{kDa}$, whereas hQC after removal of the GST-tag consists of 327 amino acids (Fig. 8.3) with a predicted molecular weight of $37.3 \mathrm{kDa}$. Although low solubility and frequent heterogeneities (e.g. chaperones like GroEL) make especially protein purification difficult, it was possible to overcome these problems by utilizing optimized affinity and anion-exchange chromatography steps. As a result, the enzyme was purified to homogeneity in the milligram scale with a yield of $0.2 \mathrm{mg}$ per one gram cell mass (Fig. 6.1). Mass spectrometry (data not shown; cooperation with Dr. Oliver Valerius from the University of Göttingen) 
and circular dichroism measurements verified successful purification of hQC. Circular dichroism spectrum of mature hQC (Fig. 6.2 A) highly corresponds to the spectra of the proteins expressed in yeast and insect cell systems [21, 85], indicating a correctly folded protein. Moreover, characterization via circular dichroism showed two minima at 208 and $222 \mathrm{~nm}$, indicating high content of $\alpha$-helices in the overall structure. Recent studies have shown that tertiary structure of hQC consists of $47 \% \alpha$-helices [21], thereby greatly differ compared to glutaminyl cyclase from Carica papaya, which adopts an all-beta fold with an $\alpha$-helix content of only $5 \%$.

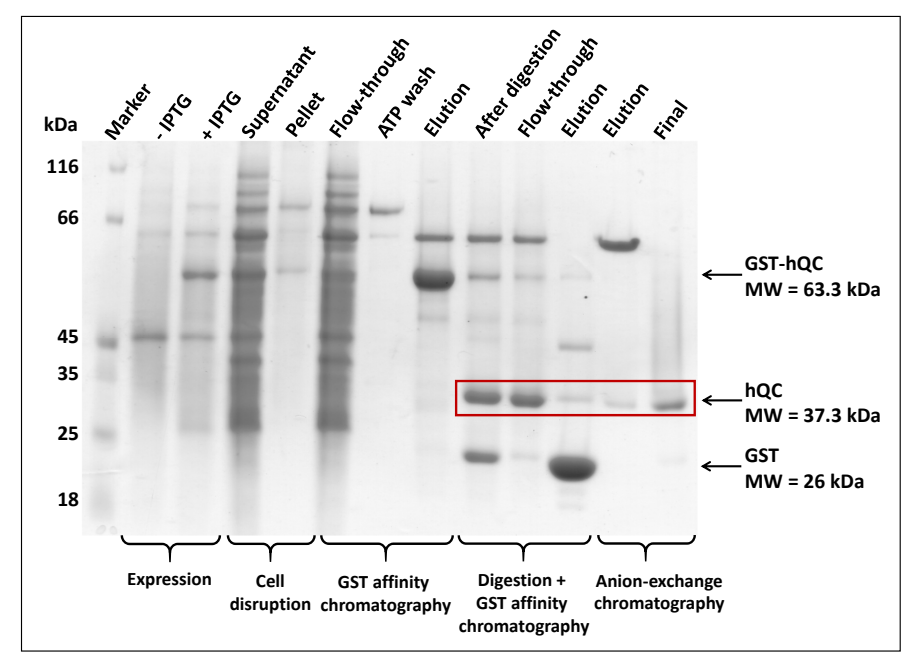

Figure 6.1: Characterization of hQC via SDS-PAGE. Overview of expression and different purification steps of hQC. Protein bands of hQC are framed in a red box. Different purification steps are indicated in brackets with a description on top of each line.

In order to obtain information about the oligomerization state of hQC, analytical ultracentrifugation was performed (data not shown; cooperation with Prof. Dr. Ute Curth from the Hannover Medical School). The results showed a main species with a sedimentation coefficient of 3.2. Based on the sedimentation coefficient value and the diffusion broadening of the sedimenting boundary layer, a molar mass of $34 \mathrm{~kg} / \mathrm{mol}$ was calculated. Since the amino acid sequence from the calculated molar mass of the monomer is 37.3 $\mathrm{kg} / \mathrm{mol}$, it can be concluded that hQC consists predominantly as a monomer in solution. Additionally, the Perrin friction factor was determined, which gives information about the deviation from a spherical shape [86] and should be for a globular protein between 1.1 and 1.2. If the Perrin friction factor differs greatly from values describing globular proteins it can be assumed that the protein structure deviates from a spherical shape, indicating a quaternary protein structure. In case of hQC a Perrin friction factor of 1.22 was deter- 
mined. This means that the protein adopts an almost perfectly spherical form, thereby supporting the idea of a soluble monomer.

To elucidate stability of hQC the enzyme was stepwise thermally unfolded starting from $293 \mathrm{~K}$ up to $369 \mathrm{~K}$ and monitored via recording the change of the far-UV CD-signal $(\lambda=196 \mathrm{~nm})$. Unfolding of hQC turned out to be an irreversible process, in which the transition showed a sigmoidal shape (Fig. 6.2 B). Data evaluation according to a sigmoidal model reveals an inflection point at $325 \mathrm{~K} \pm 4.2$. It was reported that hQC lacking the disulfide bond has a nearly identical melting point (319 K) [22]. Furthermore, recent studies showed in case of mutagenesis experiments for glycosylated mammalian glutaminyl cyclase expressed in the eukaryotic host Pichia pastoris lacking the disulfide formation, that the N-glycosylation and the zinc ion within the active site decreased thermal stability drastically $(303 \mathrm{~K})[22]$. This observation might indicate a stabilizing role of the zinc ion for the tertiary structure of hQC (see 6.4.3). In summary, missing post-translational modifications while expressing in Escherichia coli does not directly affect tertiary structure of hQC, but it influences stability and solubility, thereby explaining lower protein yield after purification. But nevertheless, hQC expressed in Escherichia coli could be used for further studies.

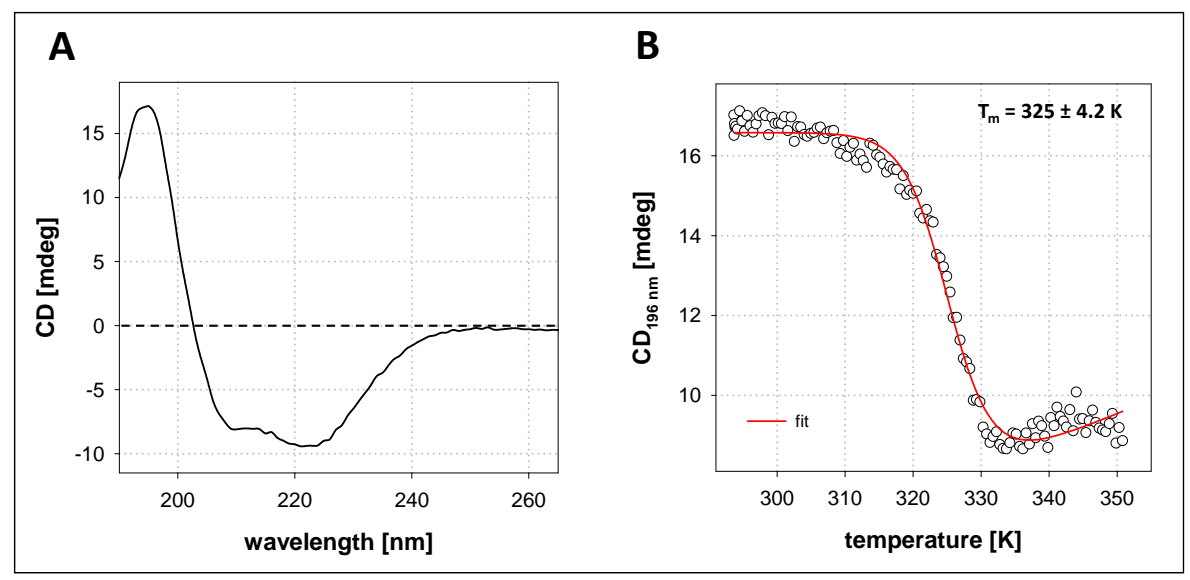

Figure 6.2: Far-UV spectra and thermal unfolding of hQC. (A) CD-spectroscopic analysis of the secondary structure of recombinant hQC. $0.1 \mathrm{mg} / \mathrm{ml}$ of hQC was dissolved in $10 \mathrm{mM}$ Tris/HCl, $\mathrm{pH}$ 8.0. (B) Thermal unfolding curve of hQC recorded by the change of the far-UV CD signal at $196 \mathrm{~nm}$. Measurement was performed in the same buffer as mentioned above. Data was fitted according to Eq. 18. 


\subsection{Development of a New Steady-State Assay}

\subsubsection{Motivation for Development of a Direct Continuous Enzymatic Assay for Human Glutaminyl Cyclase}

The cyclization reaction of N-terminal glutaminyl substrates catalyzed by glutaminyl cyclase leads to the formation of a lactam ring. Formation of the five-membered lactam ring of the side chain of glutamine leads to slight differences in the UV absorption at 210-220 $\mathrm{nm}$, which is caused by the electronic $n \longmapsto \pi^{*}$ transition of the amide bond. Those differences can be in general used to continuously study catalysis of di- and tripeptide substrates by glutaminyl cyclase. However, substrate and product of hQC have similar spectral properties, which makes it difficult to measure the cyclization reaction directly and continuously. Furthermore, due to strong absorption of other molecules at this UV/Vis range, for example peptide bonds and deoxyribonucleic acid DNA, applicability of this method is even more restricted. Therefore, hQC activity and its preference for a substrate was investigated in former studies by a previously described assay [66], which was originally based as a time-consuming discontinuous method. Due to difficulties in detecting product formation directly, a continuous coupled enzymatic assay was developed. This coupled assay [54] consists of two reactions, where in the first reaction pyroglutamic acid (pGlu) is formed by hQC and ammonia is released during the cyclization reaction. In the second reaction ammonia and $\alpha$-ketoglutaric acid are converted into glutamate by the auxiliary enzyme glutamic dehydrogenase $(\mathrm{GLDH})$ via oxidation of NADH (Fig. 5.1 B). Hence, pyroglutamyl formation catalyzed by hQC from N-terminal glutaminyl substrates is detected indirect spectroscopically at $340 \mathrm{~nm}$ by the consumption of NADH. To test if the auxiliary enzyme GLDH is not rate-limiting in the spectroscopic assay, different hQC concentrations were tested at saturated substrate conditions, while concentration of GLDH remained constant. A linear relationship was observed between the activity and the concentration of hQC for the spectroscopic coupled assay (Fig. 8.4), meaning catalysis of the auxiliary enzyme is not rate-limiting, thereby suitable for kinetic characterization of $\mathrm{hQC}$ and its various substrates. However, it was reported that hQC is also capable to catalyze the formation of pyroglutamic acid at N-terminal glutamyl substrates [3]. During cyclization of N-terminal glutamic acids a water molecule is released instead of ammonia. As a consequence, the spectroscopic assay is not suitable for kinetic characterization of 
N-terminal glutamyl substrates, due to the dependence of ammonia for the oxidation of $\mathrm{NADH}$ to $\mathrm{NAD}^{+}$in the second reaction. Thus, the spectroscopic coupled assay is limited to the detection in cyclization of N-terminal glutaminyl substrates. Therefore a direct continuous assay was tried to be developed to monitor the cyclization reaction directly for N-terminal glutaminyl-/glutamyl substrates. It should be mentioned that besides the spectroscopic coupled assay a fluorometric coupled assays was additionally developed, but it was shown to be restricted to fluorigenic substrates [87].

\subsubsection{Introduction and Overview of the Continuous Calorimetric Assay}

According to the previously mentioned arguments to develop an activity assay, isothermal titration calorimetry (ITC) turned out to be reliable method for kinetic characterization of enzymes [88] and was adopted for hQC. Generally a defined concentration of the enzyme is loaded into the syringe, whereas the substrate is placed into the sample cell of the ITC device (Fig. 6.3). After the ITC device establishes an equilibrated baseline the first injection will be performed, in which the enzyme is titrated into the sample cell and is rapidly mixed to diminish mixing artefacts. Starting with the injection heat is either released (exothermic) or consumed (endothermic), depending on the thermodynamics of the reaction. This change in heat is detected by the ITC device by measuring the decreasing/increasing differential power $(\mathrm{dp})$. The dp-value rudimentary describes the energy difference between the sample cell and a reference cell which is necessary to drive the system back to its initial state. In case of an exothermic reaction the dp increases, while for an endothermic reaction dp decreases. At the beginning of the measurement the change in dp starts to decrease/increase, which corresponds to the formation of the Michaelis-Menten complex, until it reaches a maximal stable dp-signal. At this point the enzyme is completely saturated with substrate, reflecting steady state conditions and maximal reaction velocity $\left(V_{\max }\right)$. Over time substrate is converted into product, meaning that the enzyme becomes less saturated with substrate. Thereby, heat release/consumption diminishes over time, resulting in a change of the dp-signal. In this time period the Michaelis-Menten constant $\left(K_{\mathrm{M}}\right)$ can be determined. After complete depletion of the substrate the dp-signal drives back to its initial state. Finally, data analysis and determination of kinetic parameters can be performed as already described previously (see chapter 5.2.3.2). In summary, the continuous calorimetric assay seems to be in theory a suitable tool for kinetic characteriza- 
tion of N-terminal glutaminyl-/glutamyl-substrates, because the cyclization reaction can be directly measured and is independent of optical methods.

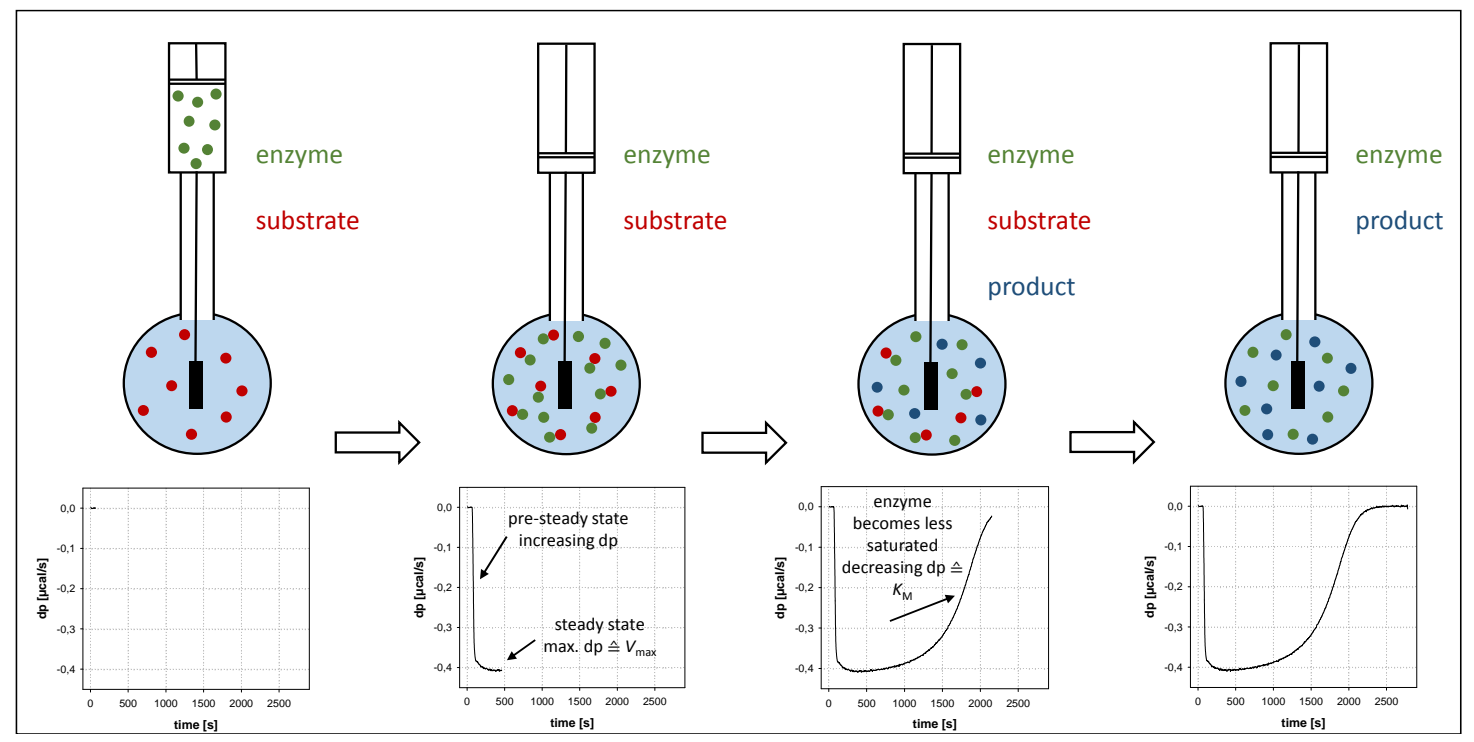

Figure 6.3: Schematic overview of the direct continuous activity assay for an exothermic reaction utilizing isothermal titration calorimetry. Sample cell and syringe of the ITC device are depicted schematically. The corresponding ITC data for each step is shown at the bottom. Arrows within the ITC data indicating important regions.

\subsubsection{First Kinetic Characterization Utlilizing Calorimetry Results in Reliable Kinetic Parameters}

To test the theory of the direct continuous calorimetric assay a kinetic characterization of hQC was performed utilizing the dipeptide Gln-Gln (QQ) as a reference substrate. Preference of a substrate for an enzyme is described by the determination of the MichaelisMenten constant $\left(K_{\mathrm{M}}\right)$, the maximal reaction velocity $\left(V_{\max }\right)$, the turnover number $\left(k_{\text {cat }}\right)$ and the catalytic efficiency $\left(k_{\text {cat }} / K_{\mathrm{M}}\right)$. These kinetic parameters were determined under steady state conditions. Therefore, conversion of Gln-Gln to pGlu-Gln (pEQ) was measured over time by monitoring the rate of heat release until complete substrate depletion. Due to the continuous monitoring of pGlu-Gln formation, turnover of hQC was measured in the dependency of different substrate concentrations in a single measurement and constants were determined as already described (see chapter 5.2.3.1). 
Figure 6.4: Conversion of the Nterminal glutaminyl reference substrate Gln-Gln catalyzed by hQC detected by calorimetry. Upper plot shows the calorimetric progress curve of the cyclization reaction of Gln-Gln, while below the corresponding Michaelis-Menten plot is depicted. After thermal equilibration (60 s), hQC was injected and the change in instrumental thermal power was monitored until substrate depletion was complete. The obtained kinetic parameters are depicted in the Michaelis-Menten plot, respectively. Measurements were performed in $50 \mathrm{mM}$ Tris/HCl, $\mathrm{pH} 8.0$, and concentrations were used according to Tab. 5.3. Obtained data points from integrated Michaelis-Menten kinetics were fitted according to Eq. 3.

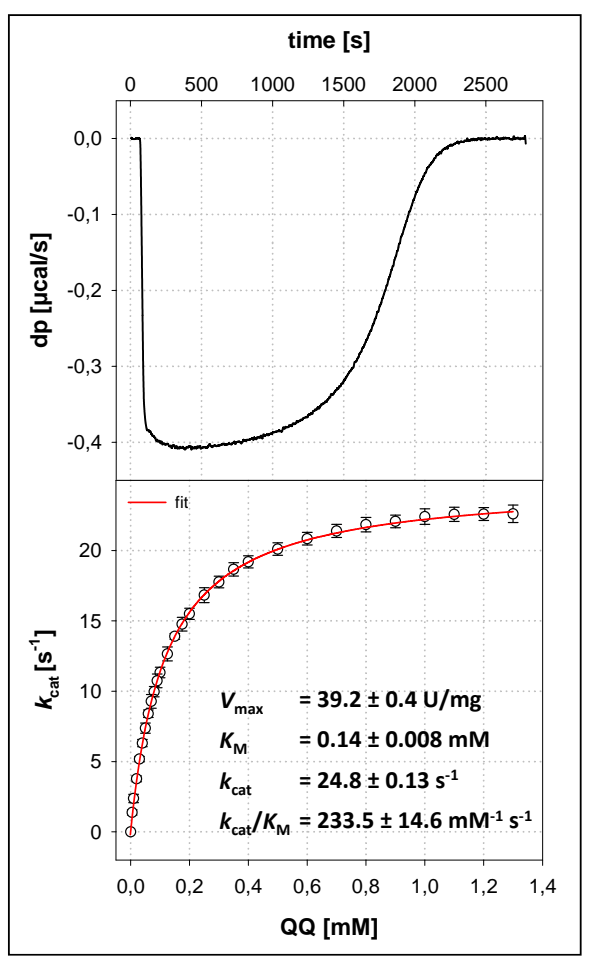

Measurements with the reference substrate resulted in a typical calorimetric curve (Fig. 6.4), as it was expected for the direct continuous calorimetric assay: After injection of hQC to the dipeptide a rapid increase in instrumental thermal power occurred. Negative dp indicating that cyclization of N-terminal glutaminyl substrates is an exothermic reaction. Afterwards, thermal power remained constant for several minutes (200-600 s), because the reaction was proceeding at maximal rate (steady state conditions). Substrate depletion caused a decrease in rate and the power returned to its initial baseline (600-2200 s). Integration of the released heat over time of the calorimetric progress curves gave a $\Delta \mathrm{H}$-value of $1.82 \mathrm{kcal} / \mathrm{mole}$, which was in turn used for calculation of rate constants (see chapter 5.2.3.1). The rate plotted as a function of the substrate concentration generated a continuous kinetic curve. Determined macroscopic kinetic constants were compared with the ones from the conventional spectroscopic coupled assay described in literature. Macroscopic kinetic constants obtained via calorimetry $\left(K_{\mathrm{M}}=0.14 \pm 0.008 \mathrm{mM}\right.$ and $k_{\text {cat }}$ $\left.=24.8 \pm 0.13 \mathrm{~s}^{-1}\right)$ were almost identical compared to the already published ones $\left(K_{\mathrm{M}}=\right.$ $0.14 \pm 0.005 \mathrm{mM}$ and $k_{\text {cat }}=20.7 \pm 0.20 \mathrm{~s}^{-1}$ [54]), which were determined spectroscopically. Data comparison of kinetic parameters revealed that kinetic characterization by calorimetry serves as an excellent alternative tool for measuring substrate preferency of hQC. However, for determination of a reliable $\Delta \mathrm{H}$-value to calculate substrate turnover 
and concentration at a given time point different prerequisites must be fulfilled, which are discussed in the following section (see chapter 6.2.4).

\subsubsection{Verification of Applicability of the Continuous Calorimetric Assay}

To characterize enzymatic reactions kinetically by calorimetry a trustworthy molar reaction enthalpy $(\Delta \mathrm{H})$ for further calculations is required. In order to obtain reliable highquality kinetic data of hQC with different N-terminal glutaminyl-/glutamyl substrates via ITC, specific prerequisites need to be fulfilled. First, the measured enzymatic reaction must be an irreversible chemical process, meaning that the change occurs only in one direction and the process will be completed. The cyclization reaction catalyzed by hQC is assumed to be an irreversible process, in which the substrate is completely converted into product over time (Fig. 8.5 A) and no equilibrium is established. Secondly, to determine an experimentally reliable enthalpy of the overall reaction the conversion of substrate into product consists of one catalysis step rather than a mixture of multiple steps. Furthermore, the process of conversion should contain the highest energy release/consumption rate. The fact that hQC catalyzes cyclization of N-terminal glutaminyl-/glutamyl substrates in a single step might indicate an experimental reliable molar reaction enthalpy. Thirdly, released/consumed heat should be independent of the enzyme and substrate concentration, if they are always used in the same ratio. In case of hQC the utilized molar ratio of enzyme to substrate was 1:2000, resulting in nearly identical $\Delta \mathrm{H}$-values (Fig. 8.5 B). Fourthly, the released/consumed heat per mole substrate should be independent of the used substrate concentration and should not deviate, which was shown for hQC (Fig. 8.5 C). Fifthly, binding of additional ligands or competitive inhibitors to the enzyme, resulting in reduced maximal reaction velocity by interfering during catalysis, should only release/consume heat marginally and thereby not influencing the overall reaction enthalpy. This could also be exhibited for the hQC system (Fig. 8.5 D). At least, the probably most important point, no product inhibition should occur, because this would distort the determined kinetic constant, especially the Michaelis-Menten constant. To show that product inhibition is negligible for hQC, the molar reaction enthalpy $\Delta \mathrm{H}$ was experimentally determined in a single measurement by measuring turnover of the dipeptide Gln-Gln catalyzed by hQC at different product concentrations (Fig. 6.5 A). For this purpose, substrate was titrated to the enzyme in the sample cell a number of times. After complete depletion 
of the substrate a new titration was started. With each titration step the concentration of the formed product increased. Measurements showed curves with nearly identical, but not perfect shapes and areas indicating a weak product inhibition. In order to further elucidate that hQC is not affected by product inhibition, data was analyzed according to Dixon [70] (Fig. 6.5 B) and by three-dimensional plotting (Fig. 8.6) to obtain the inhibitory constant $K_{\mathrm{i}}$. Binding of the product $\left(K_{\mathrm{i}}=21.4 \pm 3.1 \mathrm{mM}\right)$ is 152 -fold weaker compared to substrate $\left(K_{\mathrm{M}}=0.14 \pm 0.008 \mathrm{mM}\right)$, concluding that product inhibition is negligible. Recapitulating the facts, the continuous calorimetric assay is a reliable alternative tool compared to the spectroscopic coupled assay with the advantage to measure directly the cyclization reaction of hQC. Furthermore, the benefit of this assay is to kinetically characterize glutaminyl-, as well, glutamyl substrates.

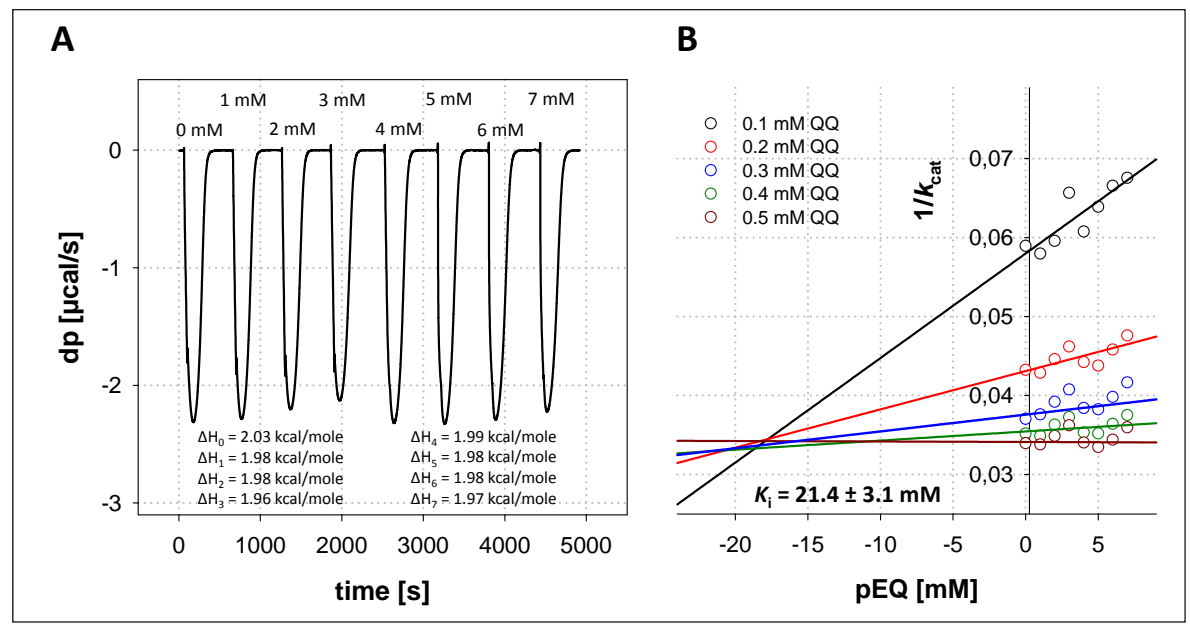

Figure 6.5: Determination of molar reaction enthalpy after complete substrate conversion in presence of different product concentrations. (A) Measurements were performed in $50 \mathrm{mM}$ Tris/HCl, pH 8.0, where $5 \mu \mathrm{l}$ of $25 \mathrm{mM}$ QQ were titrated into the sample cell containing $0.5 \mu \mathrm{M}$ hQC. With each titration step the product concentration increased continuously. Enthalpy for each titration step is shown respectively. (B) Product concentration is plotted against the reciprocal turnover of different substrate concentrations according to Dixon [70]. Data points were fitted according to a linear regression. Inhibiton constant $K_{\mathrm{i}}$ was determined by calculating the points of intersection for each linear regression.

\subsection{Kinetic Characterization of Physiologic and Pathophysiologic Substrates}

After hQC was purified to almost homogeneity in sufficient amounts and a new direct continuous enzymatic assay based on calorimetry was established, substrate specificity experiments with glutaminyl-/glutamyl substrates could be performed. Therefore, different physiological substrates harboring an N-terminal glutamine, but varying in the following amino acid sequence composition were kinetically characterized. Additionally, 
kinetic characterization of pathophysiological substrates, especially the amyloid-beta $(A ß)$ peptide, which harbors an N-terminal glutamic acid, were also characterized.

\subsubsection{Catalytic Efficiency of N-terminal Glutaminyl Substrates in Dependence of the pH}

In general, acid-base catalysis is involved in a majority of enzymatic reactions. Many amino acid side chains of catalytic sites within enzymes donate or accept protons, resulting in activation of nucleophilic and electrophilic groups, thereby facilitating the catalyzed reaction to proceed by stabilizing the charges in the transition state [89]. Functional groups that participate in acid-base catalysis are for example the alpha-amino group of an amino acid main chain or the carboxyl group of glutamic and aspartic acids. Hence, the protonation state of functional groups are $\mathrm{pH}$ dependent, enzymatic activity is sensitive to the $\mathrm{pH}$ level. To investigate the effect of $\mathrm{pH}$ on the catalytic efficiency of hQC in terms of physiological substrates, $\mathrm{pH}$ dependency experiments under constant ionic strength [69] were performed. In a further experiment for examination whether different protonation states of functional groups of the substrate or the active site of hQC have effects on catalysis, the determined macroscopic constant $K_{\mathrm{M}}$ was used as a measure for comparison.

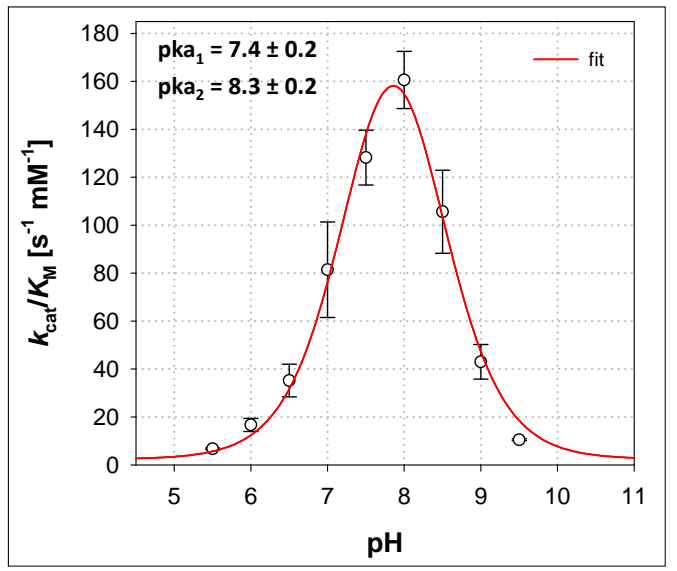

Figure 6.6: Catalytic efficiencies of hQC in dependence of the $\mathbf{p H}$. Kinetic constants were obtained employing the continuous calorimetric assay. Measurements were performed in $0.05 \mathrm{M}$ acetic acid, 0.05 M MES and 0.1 M Tris at $30{ }^{\circ} \mathrm{C}$ utilizing the reference substrate GlnGln (QQ). Data points were fitted according to Eq. 18.

Catalytic efficiency experiments in dependence of the $\mathrm{pH}$ showed a relative narrow and symmetric bell shaped curve with a maximum between 7.5 and 8.1 (Fig. 6.1). The determined $\mathrm{p} K_{a}$-values were $7.4 \pm 0.2$ and $8.3 \pm 0.2$, respectively. In addition, plotting turnover number against $\mathrm{pH}$ resulted in $\mathrm{p} K_{a}$-values of $6.6 \pm 0.2$ and $9.1 \pm 0.2$ (Fig. 8.8 A). Differences in the $\mathrm{p} K_{a}$-values of free enzyme (catalytic efficiency vs $\mathrm{pH}$ ) and the enzyme-substrate complex (turnover number vs $\mathrm{pH}$ ) lead to the assumption that the corresponding functional groups are located in the active site of the enzyme [71]. In addition 
to this reversible change of the dissociation equilibrium, a pH change could also cause an irreversible loss of enzyme activity through denaturation of the enzyme. The loss of enzyme activity due to denaturation of the enzyme could be excluded in $\mathrm{pH}$-titration experiments in case of hQC by observing no changes in the secondary structure via CD-spectroscopy (Fig. 8.8 B). However, this pH-dependent experimental results are in agreement with the already published one for hQC $\left(\mathrm{p} K_{a 1}=7.2\right.$ and $\mathrm{p} K_{a 2}=8.5$ [54]). Interestingly, the $K_{\mathrm{M}}$ values increased up to 8-fold in acidic or more alkaline $\mathrm{pH}$ values (Tab. 6.1), which might indicate that substrate affinity of N-terminal glutamines gets decreased with changing $\mathrm{pH}$-values. One possible explanation could be that the formation of the enzyme-substrate complex is dependent on the protonation state of the $\alpha$-amino group of $\mathrm{N}$-terminal glutaminyl substrates. The published value of $\mathrm{p} K_{a}$ for the $\alpha$-amino group of free glutamines in solution is 9.1 [90], which indeed fits well with the obtained pH-optimum for hQC catalyzing N-terminal glutamines. At $\mathrm{pH}$ 8.0, main species of the $\alpha$-amino group should be predominantly deprotonated, which could be a prerequisite for substrate binding. After binding of the substrate, the $\alpha$-amino group becomes nucleophilically activated for catalysis by accepting a proton from the $\gamma$-carbonyl moiety of the glutamaic acid at position 201 [55]. This could identify the $\alpha$-amino group of the substrate as an acid-base catalyst in the glutaminyl cyclase reaction, which could play an important role in binding.

Table 6.1: Determined kinetic parameters for hQC catalyzing N-terminal glutaminyl substrates in dependence of the $\mathbf{p H}$. Measurements were performed in $0.05 \mathrm{M}$ acetic acid, $0.05 \mathrm{M}$ MES and $0.1 \mathrm{M}$ Tris at $30{ }^{\circ} \mathrm{C}$. Data analysis was performed as previously described (see chapter 5.2.3.2) and kinetic parameters were determined according to Eq. 3.

\begin{tabular}{cccc}
\hline $\mathrm{pH}$-value & $k_{\text {cat }}\left[\mathrm{s}^{-1}\right]$ & $K_{\mathrm{M}}[\mathrm{mM}]$ & $k_{\text {cat }} / K_{\mathrm{M}}\left[\mathrm{s}^{-1} \mathrm{mM}^{-1}\right]$ \\
\hline \hline 5.5 & $7.57 \pm 0.70$ & $1.12 \pm 0.16$ & $6.74 \pm 0.35$ \\
6.0 & $9.56 \pm 2.88$ & $0.56 \pm 0.09$ & $16.69 \pm 2.72$ \\
6.5 & $14.38 \pm 1.15$ & $0.40 \pm 0.04$ & $35.21 \pm 6.79$ \\
7.0 & $17.97 \pm 2.71$ & $0.22 \pm 0.02$ & $81.42 \pm 19.90$ \\
7.5 & $20.65 \pm 2.09$ & $0.16 \pm 0.01$ & $128.22 \pm 11.42$ \\
8.0 & $22.47 \pm 0.13$ & $0.14 \pm 0.01$ & $160.50 \pm 11.90$ \\
8.5 & $18.47 \pm 1.88$ & $0.17 \pm 0.02$ & $105.64 \pm 17.31$ \\
9.0 & $17.26 \pm 0.15$ & $0.41 \pm 0.07$ & $43.01 \pm 7.23$ \\
9.5 & $15.18 \pm 2.95$ & $1.28 \pm 0.09$ & $10.49 \pm 0.38$ \\
\hline
\end{tabular}




\subsubsection{Kinetic Characterization with Different Physiological Substrates Indicates Importance of a Specific Amino Acid Pattern}

In order to study the catalytic mechanism of hQC and its role in metabolism a set of different N-terminal glutaminyl substrates were kinetically characterized. Thus, a detailed investigation of the impact of the nature of preliminary amino acids after the $\mathrm{N}$-terminal glutamine and the length of the substrates was performed by characterizing a number of several specifically selected physiological truncated hormones. These hormones are at physiological conditions typical substrates of hQC by modifying their N-terminal glutamine post-translationally via an intramolecular cyclization reaction. For example, the hormone Neurotensin consists of 13 amino acids [91] and plays an endocrine or paracrine role in the regulation of fat metabolism. The Gonadotropin-releasing hormone ( $\mathrm{GnRH})$, which is ten amino acids long, is responsible for secretion of luteinizing- and folliclestimulating hormones from the anterior pituitary [92]. OrexinA consists of 33 amino acids and regulates the day/night cycle and its absence would cause permanent fatigue and narcolepsy [93]. These hormones were selected for kinetic characterization via the calorimetric activity assay and represent only a small number hormones which are modified by hQC. However, all hormones differ in their amino acid sequence except for the Nterminal glutamine. The selected hormones for kinetic characterization were C-terminally truncated and consist only of the first five $\mathrm{N}$-terminal amino acids of their native sequence.

Determined kinetic constants (Tab. 6.2) and calorimetric data (Fig. 8.7) are summarized, respectively. As expected, the catalytic efficiency varies for each hormone. For instance, Neurotensin and GnRH showed comparable turnover $\left(k_{\text {cat }}\right)$, but GnRH had a 3-fold increased $K_{\mathrm{M}}$ value, suggesting lower substrate affinity. Furthermore, this effect is even more pronounced in case of OrexinA, in which the $K_{\mathrm{M}}$ value is 5 -fold increased compared to Neurotensin. The main difference between these hormones is the attribute of the subsequent amino acid after the N-terminal glutamine. In case of Neurotensin, an aliphatic amino acid at the second amino acid position is present, whereas for $\mathrm{GnRH}$ it is a polar amino acid. As recently published, substrates of hQC containing bulky and hydrophobic residues at the second and third N-terminal amino acid position are preferably converted [54], indicating hydrophobic interactions within or around the active site of the enzyme. This was pointed by a lower $K_{\mathrm{M}}$ value for substrates harboring hydrophobic and aromatic 
residues at the second and third N-terminal amino acid position, especially for phenylalanine, compared to non-hydrophobic residues. Crystal structures of hQC showed a presence of several nonpolar amino acids guiding the entrance to the substrate binding pocket, for instance Trp305 and Trp207 [20]. This observation increases the likelihood that hydrophobic interactions support the substrate binding. In addition, characterization of full length hormones [54] resulted in a 7 - to 16 -fold increased catalytic efficiency compared to the C-terminally truncated versions, caused by a 7 - to 13 -fold decreased $K_{\mathrm{M}}$ value. Thus, longer substrates seem to improve catalysis, especially substrate affinity. One possible explanation could be secondary interactions between enzyme and substrate at the protein surface of hQC, which in turn would increase binding affinity of the substrate.

In summary, one can conclude that the equilibrium between substrate association and dissociation is extensively shifted to the enzyme substrate complex for longer physiological substrates harboring bulky and hydrophobic amino acids at its second amino acid positions.

Table 6.2: Determined kinetic parameters for hQC catalyzing N-terminal glutaminyl/glutamyl substrates. Measurements were performed in $50 \mathrm{mM}$ Tris/ $\mathrm{HCl}, \mathrm{pH} 8.0$, at $30{ }^{\circ} \mathrm{C}$ via the calorimetric activity assay. Data analysis was performed as previously described (see chapter 5.2.3.2) and obtained data points were fitted according to Eq. 3 for determination of kinetic parameters.

\begin{tabular}{lcccc}
\hline Peptide & $\begin{array}{c}V_{\max } \\
{[\mathrm{U} / \mathrm{mg}]}\end{array}$ & $\begin{array}{c}k_{\text {cat }} \\
{\left[\mathrm{s}^{-1}\right]}\end{array}$ & $\begin{array}{c}K_{\mathrm{M}} \\
{[\mathrm{mM}]}\end{array}$ & $\begin{array}{c}k_{\text {cat }} / K_{\mathrm{M}} \\
{\left[\mathrm{s}^{-1} \mathrm{mM}^{-1}\right]}\end{array}$ \\
\hline \hline QQ & $39.2 \pm 0.04$ & $24.8 \pm 0.13$ & $0,14 \pm 0.01$ & $233.5 \pm 14.6$ \\
QFRH & $108.7 \pm 0.52$ & $68.6 \pm 0.32$ & $0.27 \pm 0.01$ & $254.1 \pm 29.6$ \\
EFRH $^{\mathrm{A}}$ & $0.051 \pm 0.015$ & $0.013 \pm 0.004$ & $0.15 \pm 0.02$ & $0.086 \pm 0.026$ \\
Q- $3 \mathrm{NA}$ & $32.0 \pm 1.37$ & $20.4 \pm 0.87$ & $0.12 \pm 0.01$ & $170.0 \pm 20.6$ \\
Neurotensin $_{1-5}{ }^{\text {B }}$ & $66.1 \pm 0.93$ & $48.0 \pm 1.41$ & $0.24 \pm 0.01$ & $200.0 \pm 8.3$ \\
GnRH $_{1-5}{ }^{\text {B }}$ & $77.4 \pm 2.15$ & $49.1 \pm 0.66$ & $0.69 \pm 0.04$ & $71.1 \pm 4.6$ \\
OrexinA $_{1-5}{ }^{\text {B }}$ & $17.7 \pm 0.10$ & $11.6 \pm 0.47$ & $1.28 \pm 0.05$ & $9.1 \pm 0.7$ \\
\hline A) Measurements were performed in 30 mM Tris/HCl and 20 mM MES, pH 6.8 & \\
B) Amino acid sequence: QLYEN (Neurotensin); QHWSY (GnRH); QPLPD (OrexinA)
\end{tabular}




\subsubsection{Kinetic Characterization of the Pathophysiological Substrate Shows Remarkably Decreased Catalytic Efficiency}

Cyclization of N-terminal glutamyl substrates catalyzed by hQC is very important in terms of neurodegenerative diseases, but poorly investigated to date. As already mentioned (see chapter 4.4) this pathophysiological side reaction of hQC plays a major role in the modification of $A ß$ peptides, resulting in toxic depositions within the brain $[94,95]$. Due to the pyroglutamyl formation at the N-terminus of $A ß$ peptides, these peptide species become more resistant to proteolytic degradation by aminopeptidases. In addition, hydrophobicity of modified Aß peptides are drastically increased [45], leading to aggregation and finally to toxic depositions. In order to gain deeper insights into the reaction mechanism of hQC catalyzing N-terminal glutamic acids and its kinetic parameters, the tetrapeptide EFRH was selected. The tetrapeptide EFRH represents the first four amino acids of the Aß peptide $\left(\mathrm{A}_{3-6}\right)$. To compare conversion of N-terminal glutaminyl-/glutamyl substrates kinetically, the glutamic acid was substituted against glutamine in order to obtain the tetrapeptide QFRH.

In former studies it was shown by a discontinuous assay utilizing high-performance liquid chromatography (HPLC) that the cyclization reaction of the N-terminal glutamic acid of EFRH catalyzed by hQC proceeds with a turnover number of $2.2 \times 10^{-4} \mathrm{~s}^{-1}$ [5]. Accordingly to these results it was estimated that cyclization of N-terminal glutamyl substrates is 10000-fold slower compared to N-terminal glutaminyl substrates. However, disadvantages using a discontinuous assay are that the shape of the progress curve is not readily apparent, as well as timing and volume inaccuracies might be introduced. Therefore, a first kinetic characterization of N-terminal glutamic acids catalyzed by hQC utilizing the continuous calorimetric assay (Fig. 6.7) was performed. Interestingly, calorimetric measurements with hQC and EFRH performed under alkaline conditions ( $\mathrm{pH} 8.0$ ), in which the enzyme has its $\mathrm{pH}$ optimum for cyclization of N-terminal glutaminyl substrates, no heat release was detected. However, switching to weak acidic conditions ( $\mathrm{pH}$ 6.8) heat release could be detected, which resulted in a typical progress curve suggesting turnover of EFRH. Obtained kinetic parameters showed that turnover $\left(k_{\text {cat }}\right)$ of EFRH is $\sim 5200$-fold slower compared to QFRH (Tab. 6.2), while the $\left(K_{\mathrm{M}}\right)$ value is nearly identical. Thus, catalysis of N-terminal glutamyl substrates seems dependent on the degree of the protonation state of the en- 
zyme or substrate. This theory is supported by $\mathrm{pH}$ dependency experiments of papaya QC converting Glu-ßNA utilizing a discontinuous assay [3]. It was shown that papaya QC exhibits a $\mathrm{pH}$ optimum of 6.0 for N-terminal glutamyl substrates, resulting in $\mathrm{p} K_{a}$ values of 4.2 for the $\gamma$-carboxylic moiety and 7.5 for the $\alpha$-amino group. Accordingly, oxygens of the $\gamma$-carboxylic moiety of the N-terminal glutamic acid are predominantly bivalently charged around neutral and alkaline $\mathrm{pH}$ and thereby exercising no electrophilic carbonyl activity (Fig. 6.8). Hence, switching to acidic conditions the $\gamma$-carboxylic moiety becomes protonated and the electrophilic carbonyl carbon gets nucleophilically attacked by the $\alpha$-amino group. Thus, protonation of the $\gamma$-carboxylic moiety of N-terminally glutamic acids is a prerequisite for conversion into pyroglutamic acid, which can only be achieved under acidic conditions.

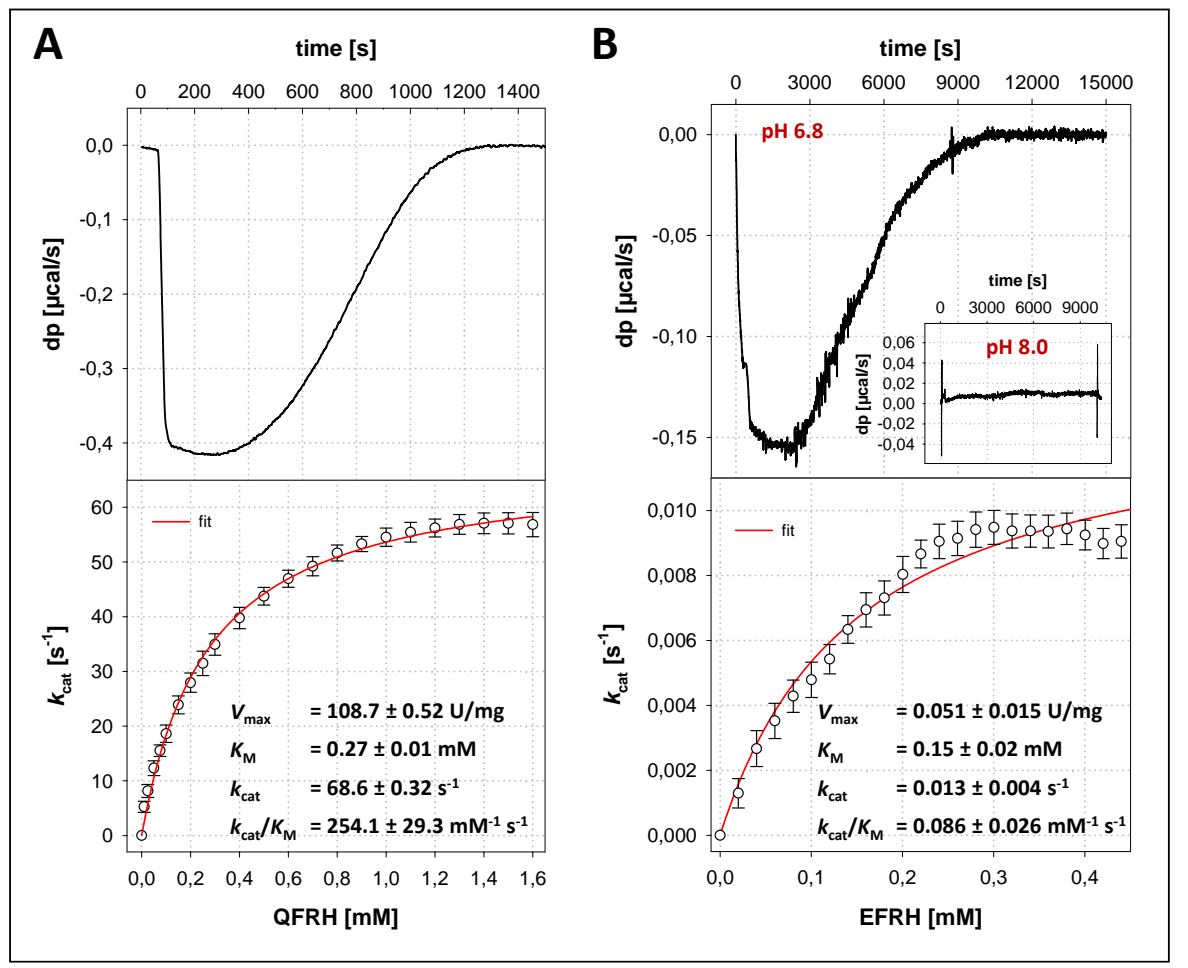

Figure 6.7: Cyclization reaction of N-terminal glutaminyl-/glutamyl substrates catalyzed by hQC. (A) Calorimetric progress curves of turnover of the tetrapeptide QFRH and (B) of EFRH $\left(\mathrm{A} \beta_{3-6}\right)$ (upper plot) with the corresponding Michaelis-Menten plot (lower plot). Measurements were performed in $50 \mathrm{mM}$ Tris- $\mathrm{HCl}, \mathrm{pH} 8.0$, in case of QFRH and $30 \mathrm{mM}$ Tris-HCl, $20 \mathrm{mM} \mathrm{MES,} \mathrm{pH} \mathrm{6.8,} \mathrm{in}$ case of EFRH. In case of EFRH, measurements were performed at alkaline and acidic $\mathrm{pH}$ conditions ( $\mathrm{pH}$ 8.0 and 6.8). The measurement at alkaline $\mathrm{pH}$ conditions showed no detectable heat release (inset), while at acidic $\mathrm{pH}$ conditions it resulted in a typical calorimetric progress curve. This observation indicates differences in the protonation states of the substrate or functional groups within the active site of hQC for catalysis of N-terminal glutamyl substrates. Data analysis of calorimetric data was performed as previously described (see chapter 5.2.3.2). Obtained data points were fitted according to Eq. 3 and determined kinetic constants are shown in the respective plots. 


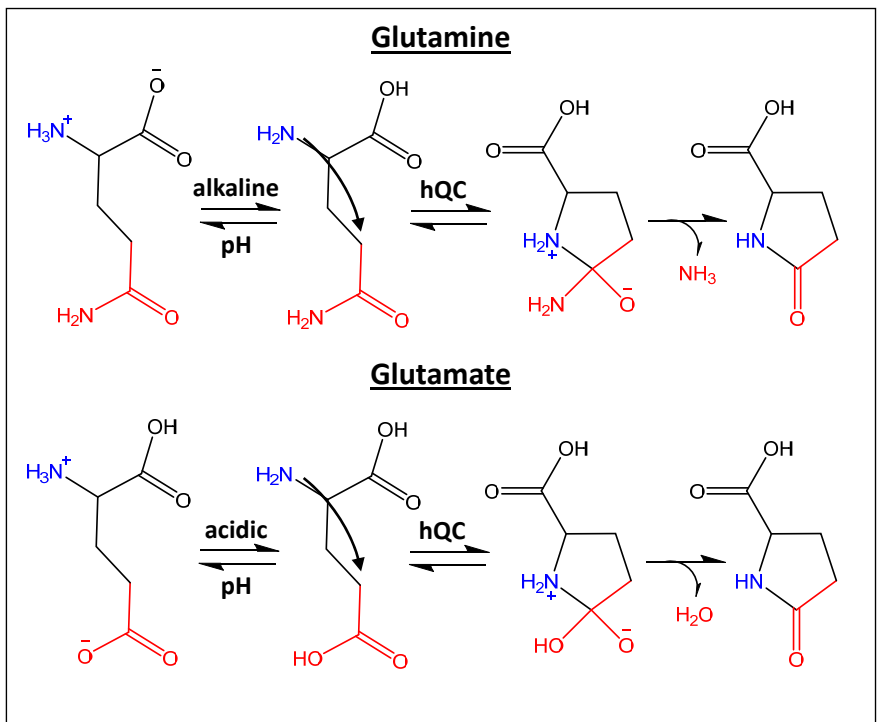

Figure 6.8: Scheme of protonation states for N-terminal glutaminyl/glutamyl substrates. In both cases an uncharged N-terminal glutaminyl/glutamyl substrate is necessary for the cyclization reaction, which is achieved at different $\mathrm{pH}$ values for each substrate. The arrow indicates the nucleophilic attack of the $\alpha$-amino group onto the electrophilic $\gamma$-carbon atom.

\subsubsection{Cyclization Reaction of N-Terminal Glutaminyl/Glutamyl Peptides are} Spatially Separated

As previously described (see chapter 6.3.3), conversion of N-terminal glutamines can only occur at alkaline $\mathrm{pH}$ conditions, whereas for $\mathrm{N}$-terminal glutamic acids an acidic $\mathrm{pH}$ value is favored. As different requirements are necessary for catalysis, the question arise how the physiological and pathophysiological cyclization reaction would be feasible within a cell. The glutaminyl cyclases of vertebrates, whose sequence apparently is highly conserved [11], are transported by a signal sequence into the endoplasmic reticulum (ER). First immunological studies for intracellular localization of the enzyme from bovine pituitary have shown that the protein is located after passage of the Golgi apparatus in secretory vesicles near the cell membrane [28]. Probably, upon the reception of an extracellular signal by the cell, glutaminyl cyclase is secreted into the extracellular space. Secretion of glutaminyl cyclase into the extracellular space is thereby mediated and controlled by the regulated secretory pathway. The $\mathrm{pH}$ of the extracellular space at physiological conditions is kept at weak alkaline $\mathrm{pH}$ conditions ( $\mathrm{pH} 7.1$ - $7.5[96]$ ), which consequently serves as the perfect environment for modification of small peptides harboring an N-terminal glutamine (Tab. 6.1). Interestingly, it was shown that modified Aß peptides harbouring an N-terminal pyroglutamyl residue and glutaminyl cyclase undergo an activity-dependent, 
regulated co-secretion from secretory vesicles, indicating conversion of the N-terminal glutamic acid into pyroglutamic acid within the secretory vesicles [97]. Due to the fact that within secretory vesicles an acidic environment predominantly exists, it would perfectly match with the findings of the performed continuous calorimetric activity assay. Cyclization reaction of N-terminal glutaminyl/glutamyl substrates might occur at different localizations. Modification of N-terminal glutamines probably takes place outside the cell, while for N-terminal glutamic acids it is likely to assume that it happens within the cell (Fig. 6.9).

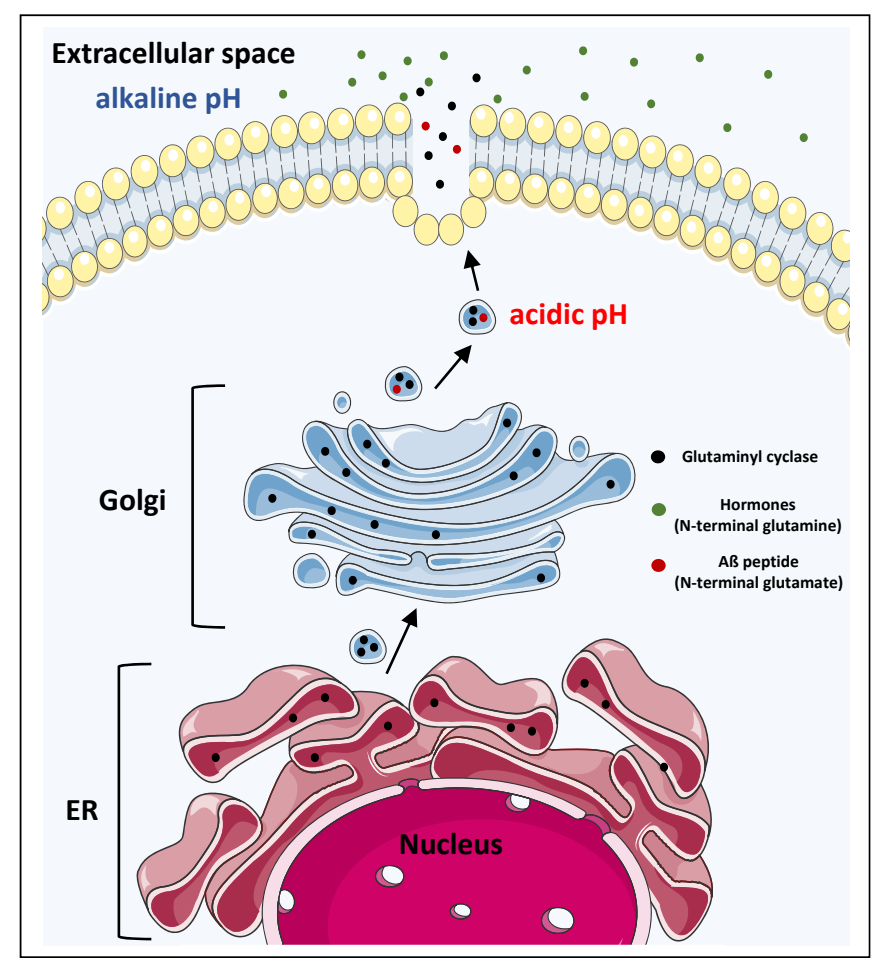

Figure 6.9: Scheme of regulated exocytosis and cyclization reaction localization of Nterminal glutaminyl/glutamyl substrates catalyzed by hQC. The enzyme is transported from the endoplasmic reticulum (ER) into the Golgi apparatus, in which the enzyme is packaged into secretory vesicles for transport to the cell membrane. At this stage $\mathrm{hQC}$ is co-localized with the unmodified $\mathrm{A} B$ peptide species within the secretory vesicles. Due to the acidic $\mathrm{pH}$ within the vesicle the $\mathrm{A} \beta$ peptide can be modified by hQC. After obtaining an external stimulus hQC is subsequently secreted into the extracellular space. Currently after secretion, hQC is co-localized with hormones and neuropeptides in the extracellular space, in which alkaline $\mathrm{pH}$ conditions exist. Under these conditions the physiological reaction of hQC can occur.

\subsubsection{Summary}

Kinetic characterization of hQC with the continuous calorimetric activity assay showed differences in the catalytic efficiencies for N-terminal glutaminyl/glutamyl substrates. Moreover, specific amino acid composition and an extended sequence length of the substrate 
seems to increase turnover and affinity. In addition, cyclization reaction of N-terminal glutamines is preferred at alkaline conditions, whereas for N-terminal glutamic acids an acidic environment is a prerequisite. Thereby it might be concluded that the protonation state of both substrates seems to play an important role in terms of binding and catalysis. In theory, necessary environmental $\mathrm{pH}$ conditions for both reactions (physiological and pathophysiological) of hQC are given within an organism.

\subsection{The Role of the Metal Ion in Terms of Stability and Catalysis}

After demonstrating that N-terminal glutamines are converted with a higher efficiency compared to glutamic acids, different methods were applied in order to get detailed information about the role of the zinc ion in hQC in terms of stability and catalysis. A closer look at the environment of the metal ion and its coordination geometry might help to elucidate the mechanism of the reaction catalyzed by hQC. Elucidation of the reaction mechanism might therefore explain the difference in catalytic efficiencies.

First, to gain deeper insights into the electronic environment and thereby into the coordination geometry of the catalytic active metal ion in absence and presence of several components including substrate, the non-spectroscopically active zinc ion within the active site of hQC was substituted against spectroscopically active cobalt. It was already demonstrated in 1958 that cobalt is a spectroscopically active surrogate for zinc [98]. However, the distorted electronically environment of the enzyme-bound cobalt monitored by a change in the UV/Vis spectra is a way to detect and visualize metal interaction with different ligands. Accordingly, various spectroscopic methods for analysis of the coordination geometry are available, including UV/Vis and stopped-flow spectroscopy, as well electron paramagnetic resonance (EPR) spectroscopy. For this purpose rapid freeze quench EPR data from Alexander Piontek of the cobalt substituted isoenzyme glutaminyl cyclase from Drosophila-melanogaster (Co(II)-isoDromeQC) in resting state and in complex N-terminal glutaminyl/glutamyl substrates [99] were additionally consulted and compared with obtained data from different applied methods for hQC. The isoDromeQC catalyzes the same cyclization reaction of N-terminal glutamines compared to hQC and both enzymes share an identical active site architecture (Fig. 8.10) due to their evolutionary orign. Therefore, obtained results from rapid freeze quench EPR experiments with isoDromeQC can be 
compared with obtained results from hQC.

Furthermore, to gather information about the structural and mechanistic features of hQC, especially coordination geometry of the zinc ion within the active site, crystal structures of hQC in resting state, in complex with N-terminal glutaminyl/glutamyl substrates and with the resulting product were generated. Data collection and refinement statistics for each crystal structure are summarized in Tab. 8.3.

\subsubsection{Substitution of Zinc Against Cobalt Resulted in a Spectroscopically Active Glutaminyl Cyclase}

For spectroscopic measurements the zinc ion was substituted against cobalt within the active site to obtain spectroscopically active hQC (Co(II)-hQC). Therefore, the catalytic zinc ion in the active site of $\mathrm{hQC}$ was removed by a dialysis step against 1,10-phenanthroline and EDTA to generate the apo-form of the enzyme. The metal chelator 1,10-phenanthroline is known to form complexes with divalent metal ions. After generation of the apoenzyme, both metal chelators were removed by a following dialysis step with Chelex-100 treated buffers. Chelex-100 is a styrene-divinylbenzene co-polymer containing iminodiacetic acid groups and has thereby the ability to bind free transitions metal ions in solution.


Figure 6.10: Absorption of $\mathrm{Co}(\mathrm{II})-\mathrm{hQC}$ in dependency of the cobalt concentration. (A) Difference spectra of Co(II)-hQC. $40 \mu \mathrm{M}$ of the prepared apoenzyme were stepwise saturated with cobalt chloride to a final concentration of $140 \mu \mathrm{M}$. Measurements were performed in Chelex-100 treated $50 \mathrm{mM}$ Tris/HCl, pH 8.0, buffer. Depicted spectra are buffer, cobalt chloride and protein corrected. (B) Determination of the dissociation constant by plotting obtained absorption intensities against cobalt concentration. Data points were fitted according to Eq. 16.

In former studies it was shown that titration of different transition metals, for instance iron or nickel, did not result in a reactivation of the enzyme [100]. However, reconstitution 
of the active site with cobalt reactivated the apoenzyme [101]. Therefore, spectroscopic active cobalt was chosen for further experiments. Coordinated transition metal centers with incomplete filled d-orbitals, e.g. cobalt, can be excited by light. Transitions of electrons from low energy d-orbitals to high energy d-orbitals ('d-d' transitions) due to absorption of light of a certain wavelength should result in a specific and distinct absorption pattern. Excited and coordinated cobalt absorbs light at three different wavelength and should show consequently three characteristic peaks [102]. As expected, titration of cobalt ions to the apoenzyme showed characteristic absorption signals at 520, 550 and $580 \mathrm{~nm}$, which became more pronounced with increasing cobalt concentration until the enzyme was completely saturated (Fig. 6.10 A). At a molar ratio of $1: 3$ (hQC : cobalt) the maximum signal was obtained, suggesting complete saturation of the enzyme. Thus, cobalt was successfully incorporated within the active site of hQC.



Figure 6.11: Time-resolved absorption spectra and activity assay of Co(II)-hQC. All measurements were performed in $50 \mathrm{mM}$ Tris/HCl, pH 8.0 and used buffer was Chelex-100 treated before measurements to remove free transition metals. (A) Absorption spectra of $\mathrm{Co}$ (II)-hQC measured at defined time points. $40 \mu \mathrm{M}$ apoenzyme was supplemented with $144 \mu \mathrm{M}$ and spectra were recorded step wise in a time range from 0 - 60 minutes. Depicted spectra are buffer, cobalt and protein corrected. (B) Progress curves of $\mathrm{Zn}(\mathrm{II})-\mathrm{hQC}$, Co(II)-hQC and the apoenzyme measured with the spectroscopic coupled assay. Turnover number for each enzyme form is depicted respectively.

To test if the coordinated cobalt within the active site of hQC forms a stable complex, $40 \mu \mathrm{M}$ apoenzyme was supplemented with $144 \mu \mathrm{M}$ cobalt chloride and absorption spectra were recorded at different time points (Fig. 6.11 A). Decreasing absorption signal intensities of the characteristic absorption bands of $\mathrm{Co}(\mathrm{II})$-hQC could not be observed, indicating a stable complexation of the cobalt within the active site of hQC. In addition, after four minutes the dissociation equilibrium towards complex formation was nearly completed. Besides a stable complex formation the substituted cobalt should show comparable activ- 
ity compared to zinc. Therefore, enzyme activity of hQC and Co(II)-hQC were measured with the spectroscopic coupled assay and compared to each other (Fig. 6.11 B). Additionally, the apoenzyme was also measured as a control. As expected, hQC and Co(II)-hQC showed nearly the same catalytic activity, implying full reactivation of the apoenzyme by cobalt. The apoenzyme showed almost no catalytic activity and had only $0.01 \%$ residual activity compared to the holoenzyme, verifying successful removal of the catalytic zinc ion from the active site of hQC. In summary, replacement of the diamagnetic zinc against the chromophoric and paramagnetic cobalt provides a powerful spectral probe of the active site environment while maintaining enzymatic activity.

\subsubsection{Incorporated Cobalt Within the Active Site of Human Glutaminyl Cyclase Shows a Moderate Tetrahedrally Coordination}

In order to obtain information about the stability of the binding of cobalt within the active site of hQC the equilibrium dissociation constant $\left(K_{\mathrm{d}}\right)$ was determined. The $K_{\mathrm{d}}$ value describes the propensity of a larger macromolecule to dissociate reversibly into its smaller components. Accordingly, a lower $K_{\mathrm{d}}$ value would mean that the equilibrium is shifted towards the complex formation, meaning in general stronger and tighter binding of the components to each other. Complexation of cobalt and hQC to $\mathrm{Co}(\mathrm{II})-\mathrm{hQC}$ is a simple bi-molecular association with a stoichiometry of $1: 1$. For $K_{\mathrm{d}}$ determination multiple standardized reconstitution activity reactions were performed (Fig. 8.9) and additionally cobalt was stepwise titrated to apoenzyme (Fig. 6.10 A). The apparent $K_{\mathrm{d}}$ value was determined by plotting turnover number against cobalt concentration (Fig. 6.10 B). Fitted data points resulted in an apparent $K_{\mathrm{d}}=28.7 \pm 3.9 \mu \mathrm{M}$. As published previously, a computational designed high-affinity Zn(II)-binding site with a predetermined tetrahedral geometry and alternating zinc binding motifs resulted in $K_{\mathrm{d}}$ values in the nanomolar to micromolar range [103]. Substitution of the tetrahedral coordinated zinc against cobalt within the high-affinity $\mathrm{Zn}(\mathrm{II})$-binding site showed 2 - 3 orders of magnitude weaker binding. $K_{\mathrm{d}}$ values of $2-4 \mu \mathrm{M}$ were defined as strong binding, while $150-250$ $\mu \mathrm{M}$ were classified as weak binding [104]. Therefore, the experimentally determined $K_{\mathrm{d}}$ value for cobalt towards hQC perfectly matches between both classifications and binding can be considered as moderate. Presumably, zinc binding within the active site of hQC is stronger compared to cobalt due to the presence of specific amino acids in the first 
coordination sphere. It was shown that cysteine residues in the first coordination sphere are stronger determinants for cobalt binding compared to histidine residues, for example [104]. The negative charge on the thiolate of the cysteine residue is known to provide a stronger binding contribution.

\subsubsection{Coordinated Zinc Ion Plays a Role in Stabilization of the Overall Structure of Human Glutaminyl Cyclase}

A wide variety of metal ions are found within the human body. Many of these divalent metal ions form complexes with proteins, in which they possess various functions. One of these metals is zinc, which is an essential component of many enzymes involved in virtually all aspects of metabolism. The roles of zinc in enzymes have been implicated in intermolecular interactions with other macromolecules such as protein and DNA, polypeptide folding, conformational stability, substrate binding and catalysis [105, 106]. As described previously, human glutaminyl cyclase was already identified as a metalloenzyme harboring a catalytic active zinc ion within its active site [23]. Typically, catalytic zinc ions are coordinated by three amino acids, which are in case of hQC Asp159, Glu202 and His330 (see chapter 6.4.4). Tridentate combinations of histidine, cysteine, glutamic and aspartic acid side chains have been described to bind strongly to zinc ions and a characteristic of catalytic function is to provide a free coordination site for water or substrates [107]. To investigate if the zinc ion plays a role in conformational stability, thermal unfolding curves of the apoenzyme, hQC and Co(II)-hQC were measured.

All thermal unfolding curves showed a transition with a sigmoidal shape (Fig. 6.2 B and 6.12). After thermal unfolding a CD spectrum of each enzyme form was measured to ensure complete denaturation (Fig. 8.11). As already assumed, hQC harboring zinc in its active site showed the highest thermal stability $\left(\mathrm{T}_{m}=325 \pm 4.2 \mathrm{~K}\right)$ compared to other enzyme forms, whereas the apoenzyme exhibited the lowest thermal stability $\left(\mathrm{T}_{m}=312 \pm\right.$ $3.5 \mathrm{~K}$ ). These findings pointing to a structural destabilization of the enzyme as a result of the absence of a metal ion. Interestingly, reconstitution of hQC with cobalt did not result in same thermostability for the enzyme compared to zinc $\left(\mathrm{T}_{m}=318 \pm 4.1 \mathrm{~K}\right)$. Cobalt substitution considerably lowered the thermostability of the enzyme without affecting the overall conformation, suggesting that the prosthetic zinc is affecting both conformational 
stability as well as catalysis. Probably, the zinc coordinating residues of hQC are provided from three different loop regions of the enzyme. Binding of the zinc within the active site by the zinc coordinating residues could increase the stability of the loops and thereby the overall structure. Coordination of cobalt is not as strong as the coordination of zinc (see chapter 6.4.2), explaining the lower thermostability of $\mathrm{Co}(\mathrm{II})-\mathrm{hQC}$.



Figure 6.12: Thermal unfolding of Co(II)-hQC and apoenzyme. Thermal unfolding curves were recorded by the change of the far-UV CD signal at $196 \mathrm{~nm}$. Measurements were performed in 10 $\mathrm{mM}$ Tris/ $\mathrm{HCl}, \mathrm{pH} 8.0$ with a protein concentration of $0.1 \mathrm{mg} / \mathrm{ml}$. Thermal unfolding curve of (A) the apoenzyme and (B) $\mathrm{Co}(\mathrm{II})-\mathrm{hQC}$ are shown respectively. Data points were fitted according to Eq. 18.

\subsubsection{Zinc Ion Within the Active Site of hQC in Resting State Shows a Tetrahedral Coordination}

To elucidate the coordination geometry of the coordinated zinc within the active site of hQC in resting state different methods were applied. As already described previously, catalytic zinc ions of metalloenzymes are tetrahedrally coordinated by three residues and one water molecule, which acts as a place holder and gets displaced in the presence of substrate [107]. To verify if the zinc ion within the active site comply the typical structural conditions for a catalytic metal ion, X-ray crystallography was attempted for hQC in resting state to get insights into the tertiary structure of hQC. The main focus was set on the active site of hQC with its coordinated metal ion.

The enzyme crystallized within the space group R32 and crystals diffracted with a resolution of $2.14 \AA$. The asymmetric unit comprised two autonomic molecules, which stands in agreement with the obtained analytical ultracentrifugation experiments (see chapter 6.1). It was shown via analytical ultracentrifugation that hQC consisted as a monomer in solu- 
tion. Both molecules in the asymmetric unit were identical in their overall structure. The monomeric globular structure with dimensions of 49 × 51 x $41 \AA^{3}$ revealed a mixed $\alpha / \beta$ fold with an open-sandwich topology. The hydrophobic core of the enzyme consists of a central twisted six-stranded $\beta$-sheet, formed by two antiparallel and four parallel strands. This central six-stranded $\beta$-sheet is encircled by six and two $\alpha$-helices on opposite sides. Hence, the overall structure of hQC perfectly matches with the already published crystal structure of hQC in resting state [20].

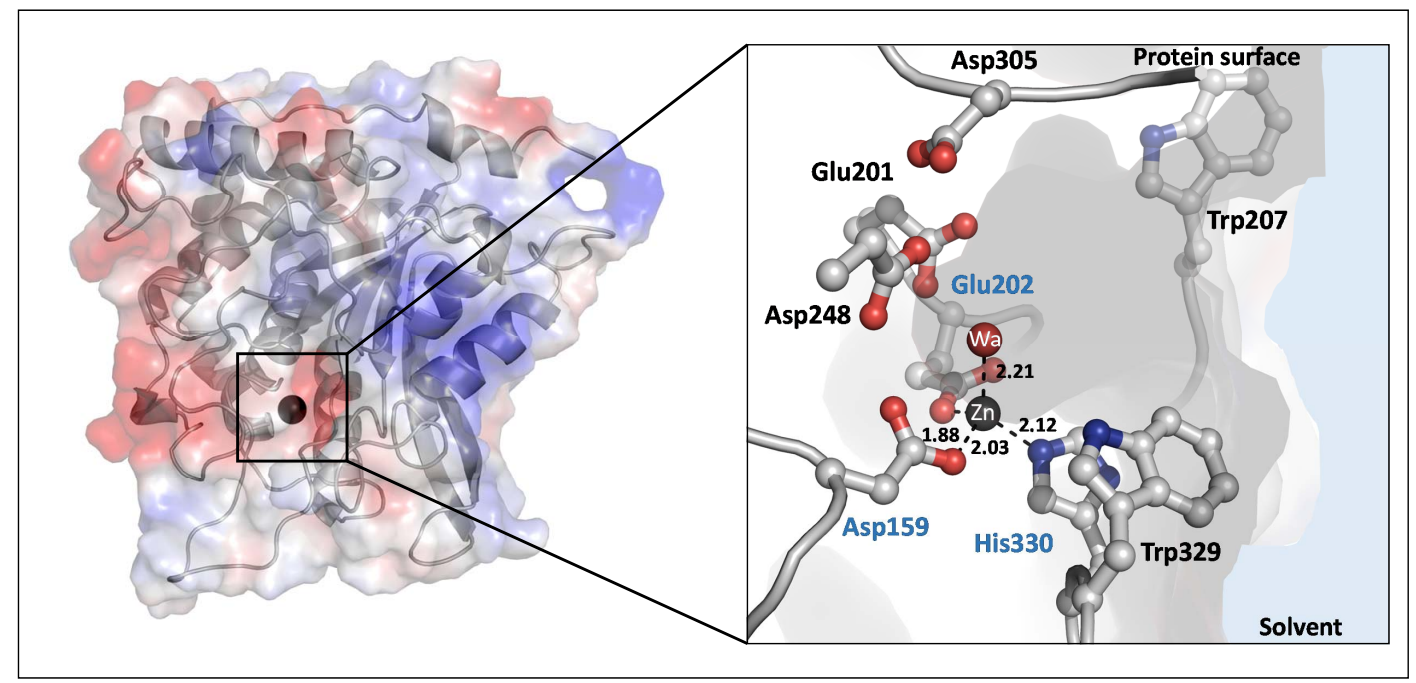

Figure 6.13: Overview of the overall crystal structure of hQC in resting state with a detailed side view of the active site. Secondary structures of hQC are presented in cartoon style, while the protein surface with its electrostatic potentials is indicated transparently. Red regions indicating negatively charged regions, whereas blue regions are positively charged. Zinc ion is represented as a black sphere. The active site is located at the protein surface of the enzyme, as indicated in the enlargement. Hydrophobic pocket is represented in transparent gray. Important residues are depicted and named respectively, while zinc coordinating residues are highlighted in blue. Coordinated water molecule is shown as a red sphere. Dashed lines indicate the coordination bonds with the corresponding distances in angstroms. Resolution $=2.14 \AA$, R-work $=15.7 \%$ and $\mathrm{R}$-free $=19.7 \%$

A detailed view into the hydrophobic active site pocket of hQC revealed that the metal ion is tetrahedrally coordinated (Fig. 6.13). It is located in a cavity at the protein surface with a depth of approximately $8 \AA$, while the entrance is guided by two hydrophobic residues, namely Trp207 and Trp329. Furthermore, the metal ion is coordinated by an imidazole group (His330) and two $\gamma$-carboxylate moieties (Asp159 and Glu202). As expected for catalytic zinc ions, the fourth coordination site is occupied by a water molecule. Coordination bond distances and angles for each atom of the tetrahedral complex are summarized in Tab. 8.1 and Tab. 8.2, respectively. Statistical data bank analysis of zinc coordinating metalloenzymes by X-ray crystallography and nuclear magnetic resonance spectroscopy revealed common distances for specific first coordination sphere residues [108]. A Typical 
coordination bond length between a zinc ion and an aspartate residue is $2.10 \pm 0.24 \AA$, for a glutamic acid residue $1.83 \pm 0.16 \AA, 2.09 \pm 0.14 \AA$ for a histidine residue and in case of a water molecule $2.18 \pm 0.03 \AA$. This is in agreement with the observed bond distances between the zinc ion and the corresponding coordinating residues in the crystal structure of hQC in resting state. In addition, angles differ marginally compared to an ideal symmetrical tetrahedron with perfect angles of $109.5^{\circ}$. Thus, obtained UV/Vis difference spectra of $\mathrm{Co}(\mathrm{II})-\mathrm{hQC}$ in resting state with maxima at 520, 550 and $580 \mathrm{~nm}$ can be assigned as a typical tetrahedral coordination (Fig. 6.10 A).

Besides the first coordination sphere residues His330, Glu202 and Asp159 are equally important residues in the second coordination sphere, namely Glu201, Asp248 and Asp305. The acidic residues are pointing to each other and forming a hydrogen bond network, which was shown to be crucial for catalysis. Site-directed mutagenesis experiments showed a drastically loss of activity by the substitution of only one of the hydrogen bond network forming residues in the active site [55]. This effect can be explained by the interruption of the hydrogen bond network, in which the enzyme loses the capability to transfer a proton onto the leaving group of the tetrahedral reaction intermediate. In summary, the zinc ion shows all characteristics to be a catalytic metal ion [107] with nearly perfect bond distances and angles and with a conserved hydrogen bond network in its second coordination sphere.

\subsubsection{Monodental Binding of N-terminal Glutaminyl Substrates Results in a Tetrahedral Coordination}

To elucidate the role of the metal ion and its coordination geometry in terms of catalysis for the physiological and pathophysiological reaction, crystal structures of hQC in complex with N-terminal glutaminyl/glutamyl substrates were generated. In addition, stopped flow UV/Vis spectroscopy experiments were performed. The tetrapeptides QFRH (glutaminyl substrate) and EFRH (glutamyl substrate, $\mathrm{A}_{3-6}$ ) were utilized as substrates. This chapter describes the coordination geometry of the catalytic zinc in presence of the N-terminal glutaminyl substrate. As mentioned in the introduction the N-terminal glutaminyl cyclization reaction correlates to the physiologic function of hQC. The physiological reference peptide of the $\mathrm{AB}_{3-6}$ peptide QFRH exhibited in the kinetic characterization experiments the highest turnover and catalytic efficiency in the continuous calorimetric activity assay 
compared to the other physiological substrates (Tab. 6.2). This observed effect probably results from the amino acid residue composition at the $\mathrm{N}$-terminal second and third amino acid position of the tetrapeptide. As already discussed previously, bulky hydrophobic amino acids, especially phenylalanine, increase substrate affinity. For examination of the coordination geometry of the catalytic zinc ion by binding of physiological substrates and its influence in catalysis, the tetrapeptide QFRH was always utilized in all experiments and served as a representative for physiological substrates.

To get first insights into the coordination geometry of the substrate-metal complex in $\mathrm{Co}(\mathrm{II})-\mathrm{hQC}$, stopped flow UV/Vis spectroscopy was performed. In order to monitor timedependent spectroscopic changes at different wavelengths simultaneously, a photo diode array detector was used. As expected, upon rapid mixing of $\mathrm{Co}(\mathrm{II})$-hQC with the tetrapeptide QFRH strong changes in the absorption bands, especially at $525 \mathrm{~nm}$ and $555 \mathrm{~nm}$ could be observed (Fig. 6.14). Obtained stopped flow UV/Vis absorption spectra were not corrected from spectral interferences of free cobalt ions in solution. This is probably the reason why the obtained difference spectra distinguishes slightly from the UV/Vis difference spectra of $\mathrm{Co}(\mathrm{II})$-hQC in resting state (Fig. $6.10 \mathrm{~A}$ ), particularly in the $480-530 \mathrm{~nm}$ range. The intensity of the absorption signal increased over time after rapid mixing of both reactants. This observation might indicate that substrate molecules bind to the cobalt ion and form the Michaelis-Menten complex followed by the irreversible cyclization reaction. After $50 \mathrm{~s}$ the absorption signal started to lose intensity. At this time point the enzyme is apparently getting less saturated due to substrate turnover. The substrate was completely consumed between 50 and $100 \mathrm{~s}$, which correlates with rough estimations based on the time-dependent depletion of QFRH catalyzed by hQC monitored in the continuous calorimetric activity assay (Fig. 6.7 A). However, the absorption signal did not went back to its initial state after complete substrate depletion. A possible explanation might be that the resulted product also coordinates to the metal ion. Since stopped flow UV/Vis absorption spectra of $\mathrm{Co}(\mathrm{II})$-hQC in resting state and in complex with the tetrapeptide QFRH showed same absorption band characteristics, it might indicate that the hQC-substrate complex is also tetrahedrally coordinated. This idea is supported by EPR experiments with Co(II)isoDromeQC (data from Alexander Piontek) [99]. In rapid freeze quench EPR experiments $\mathrm{Co}(\mathrm{II})$-isoDrome $\mathrm{QC}$ were mixed together with QFRH and rapidly freeze quenched after 
$20 \mathrm{~ms}$ to fix the state of bound substrate towards the metal ion. Thereby, differences in the cobalt coordination sphere induced by binding of the N-terminal glutaminyl substrate were analyzed. EPR spectra showed a shift in the binding of QFRH to the lower magnetic field from $1038 \mathrm{G}$ (resting state) to $1024 \mathrm{G}$ (enzyme substrate complex) (Fig. 6.18). Band width of the resonance signal is narrowed and did not differ from $\mathrm{Co}(\mathrm{II})$-isoDromeQC in resting state, indicating a four-fold coordination. In summary, these results support the idea of a tetrahedral coordinated metal ion in presence of physiological substrates.

Figure 6.14: Time resolved UV/Vis absorption spectra of $\mathrm{Co}(\mathrm{II})-\mathrm{hQC}$ while catalyzing the tetrapeptide QFRH. For measurements a final concentration of $109 \mu \mathrm{M} \mathrm{Co(II)-}$ hQC and $8 \mathrm{mM}$ QFRH after mixing was utilized. Arrows indicating the change of absorption over time. Scheme of the coordination geometry should elucidate how binding of N-terminal glutaminyl substrates to the originally zinc might look like. Measurements were performed in 50 $\mathrm{mM}$ Tris/ $\mathrm{HCl}, \mathrm{pH} 8.0$, at $10{ }^{\circ} \mathrm{C}$. Used buffer was Chelex-100 treated before measurements. Depicted spectra are buffer and protein corrected.



To confirm obtained results from stopped flow UV/Vis spectroscopy a detailed structural view into the active site of $\mathrm{hQC}$ in complex with the N-terminal glutaminyl substrate was applied. Crystallization attempts with QFRH and hQC were successfully and resulted in analyzable data. However, only the N-terminal glutamine of the tetrapeptide QFRH could be modeled into the corresponding mFo-DFc electron density map, whereas the other amino acids had no defined electron density and were omitted. High flexibility of the hydrophobic tail of the tetrapeptide, which is directly exposed towards the solvent, is probably the reason for the missing signal. As expected, the N-terminal glutamine of the tetrapeptide bound monodentally to the catalytic zinc ion, resulted in a tetrahedrally coordination geometry of the metal ion. This observation is supported by data from stopped flow UV/Vis and EPR spectroscopy experiments with $\mathrm{Co}(\mathrm{II})-\mathrm{hQC}$ and $\mathrm{Co}(\mathrm{II})$ isoDromeQC. The enzyme-substrate complex showed a coordinative bond lengths of 2.10 $\AA$ between the zinc ion and the $\gamma$-oxygen atom of the side chain of the glutamine. It was published that a coordinative bond between a zinc ion and an oxygen atom should have a distance of $2.20 \pm 0.13 \AA$ [108]. Therefore, the coordinative bond length between the catalytic zinc of hQC and the $\gamma$-oxygen of the substrate in the crystal structure is slightly shortened, but still acceptable. Coordination bond lengths and angles for the coordinating 
residues, which are summarized in Tab. 8.1 and Tab. 8.2, respectively, showed no remarkably differences compared to the resting state structure of hQC. Statements of additional interactions between the substrate and active side residue like hydrogen bonds could not been made due to the missing residues of the tetrapeptide in the crystal structure. In summary, crystal structure of hQC in complex with the tetrapeptide QFRH confirmed observations made in stopped flow UV/Vis spectroscopy with $\mathrm{Co}(\mathrm{II})-\mathrm{hQC}$ and freeze quench EPR experiments with $\mathrm{Co}(\mathrm{II})$-isoDromeQC. N-terminal glutaminyl substrates bind monodentally to the catalytic zinc ion, which results in a tetrahedral coordination geometry for the central metal ion.

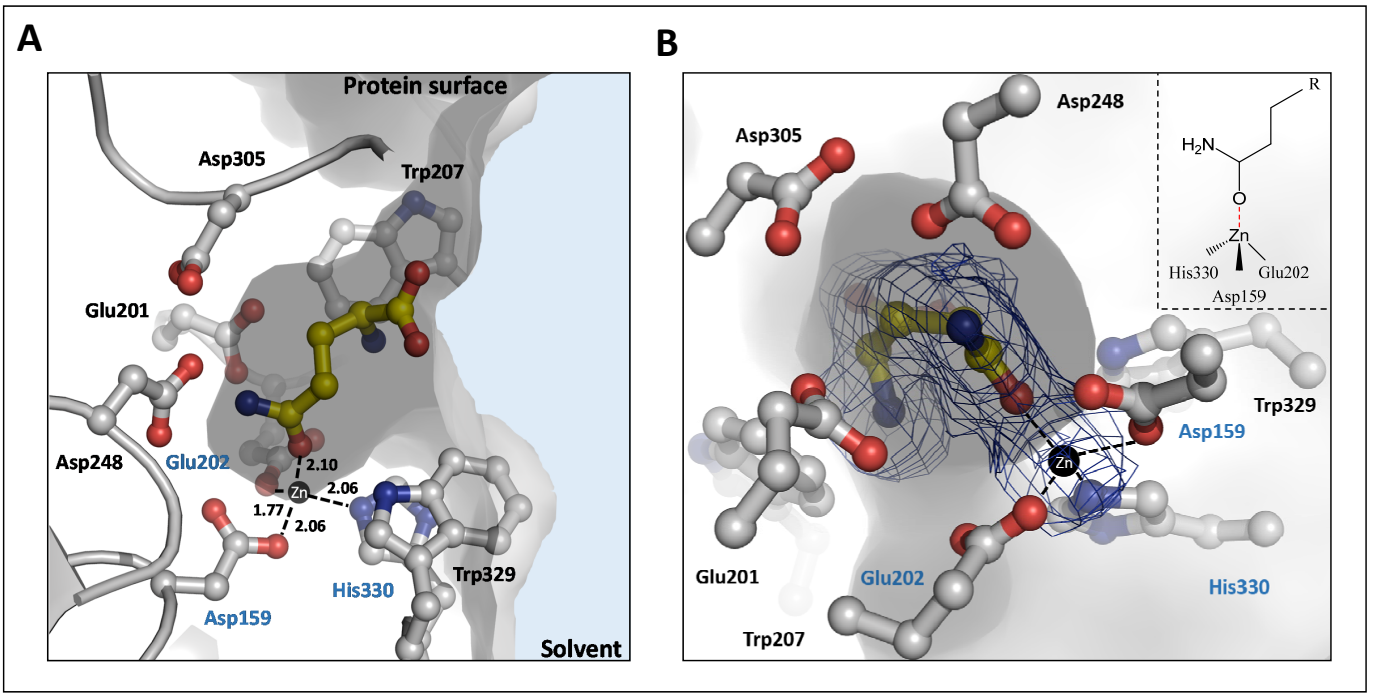

Figure 6.15: Crystal structure of hQC in complex with the tetrapeptide QFRH. (A) Side view of the active site of hQC with the coordinated substrate QFRH. Active site residues are shown in grey and named respectively (blue $=$ coordinating residues, black $=$ active site residues), while the substrate is colored yellow. The hydrophobic pocket is indicated in transparent gray, in which the coordinated zinc (black sphere) is located at the bottom of the pocket. Dashed lines are indicating coordinating bonds with the corresponding distance in $\AA$. (B) Front view of the active site with the corresponding $2 \mathrm{mFo}-\mathrm{DFc}$ electron density map with a sigma contour level of $1.0 \mathrm{\sigma}$. Scheme in the upper right corner should indicate coordination of the metal ion. Resolution $=1.90 \AA$, R-work $=17.9 \%$ and R-free $=21.5 \%$

\subsubsection{The Cyclization Product of N-terminal Glutaminyl/Glutamyl Substrates Binds monodentally to the Catalytic Zinc Ion}

As already discussed in the stopped flow UV/Vis spectroscopy experiments of $\mathrm{Co}(\mathrm{II})$ hQC catalyzing the cyclization reaction of the N-terminal glutamine of the tetrapeptide QFRH, the resulting pyroglutamyl product (pEFRH) could possibly occupy the fourth coordination site of the catalytic zinc ion (see chapter 6.4.5). The absorption band did not return back into its initial state after the substrate was completely converted into 
product, indicating coordination of the product towards the metal ion. Additionally, band characteristics were nearly identical compared to the spectrum of the enzyme in resting state, suggesting a monodental binding of the product. This theory is supported by freeze quench EPR experiments with the $\mathrm{Co}(\mathrm{II})$-isoDromeQC and the product pEFRH, in which the spectrum showed a narrow peak at $1017 \mathrm{G}$ similar to the measured EPR spectra of the enzyme in resting state and in complex with QFRH (Fig. 6.18). These observations might indicate that the formed product binds monodentally to the catalytic zinc ion, resulting in an enzyme-product metal complex with a tetrahedral coordination geometry.

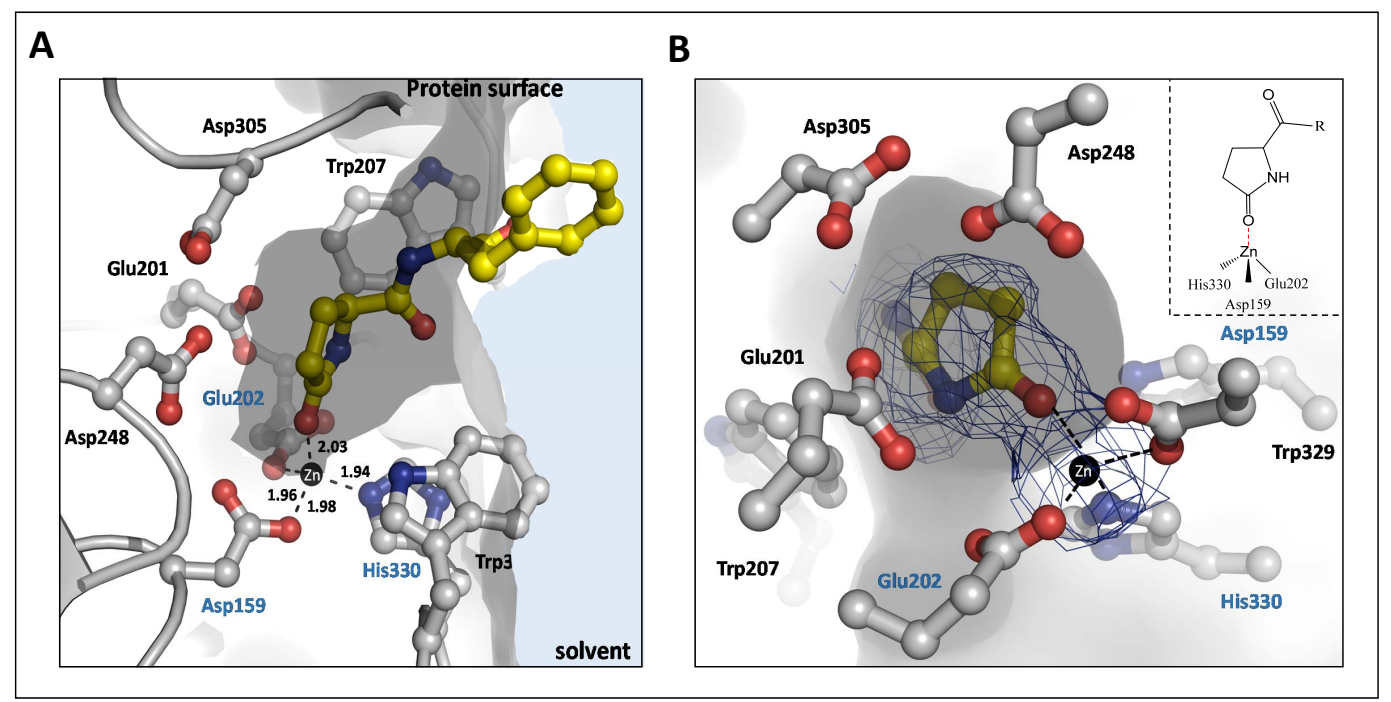

Figure 6.16: Crystal structure of hQC in complex with the product pEFRH. (A) Side view of the active site of hQC with the coordinated product pEFRH. Active site residues are shown in grey and named respectively (blue $=$ coordinating residues, black $=$ active site residues), while the product is coloured yellow. The hydrophobic pocket is indicated in transparent gray, where the coordinated zinc (black sphere) is located at the bottom of the pocket. Dashed lines are indicating coordinating bonds with the corresponding distance in $\AA$. (B) Front view of the active site with the corresponding $2 \mathrm{mFo}-\mathrm{DFc}$ electron density map at $1.0 \sigma$. Scheme in the upper right corner should indicate coordination of the metal ion. Resolution $=1.88 \AA$, R-work $=15.3 \%$ and $\mathrm{R}$-free $=17.8 \%$

To gather information if the product forms a coordinative bond with the zinc ion within the active site, which would typically results in a tetrahedron, crystal structures of hQC in complex with pEFRH were applied. Again, crystallization attempts were successfully and analyzable data of hQC in complex with pEFRH were achieved. Analysis of the active site of the crystal structure of hQC in complex with pEFRH showed a defined electron density for the N-terminal pyroglutamic acid and the adjacent phenylalanine, allowing to model both residues in the corresponding mFo-DFc electron density map. As expected, the crystal structure confirmed coordination of the product at the fourth coordination site of the catalytic zinc ion (Fig. 6.16). The keto-oxygen of the N-terminal 
pyroglutamic acid displaced the water molecule and occupied the fourth coordination site, thereby maintaining the tetrahedrally coordinated character of the zinc. Bond lengths for each coordinating residue and corresponding angles of the tetrahedron are summarized in Tab. 8.1 and Tab. 8.2, respectively. Structural data bank analysis of X-ray crystallography and nuclear magnetic resonance spectroscopy of zinc coordinating ligands showed that a zinc-oxygen bond distance should be $2.20 \pm 0.14 \AA$ [108]. Thus, the bond length between the keto-oxygen of the N-terminal pyroglutamic acid and zinc $(2.03 \AA)$ seems to be shortened. In addition to the coordination bond a potential hydrogen bond candidate was found, which could be formed between Phe2/N of the product and Gln304/O of the enzyme. This hydrogen bond might stabilize the hydrophobic tail of the product and makes it more rigid, which could explain the observed electron density of the phenylalanine. In conclusion, the resulted product after the N-terminal cyclization reaction of $\mathrm{N}$-terminal glutaminyl/glutamyl substrates is able to coordinate to the catalytic zinc ion and thereby maintaining the tetrahedral coordination geometry.

\subsubsection{Coordination of the N-terminal Glutamyl Substrate Revealed a Carboxylate Shift}

After demonstrating the tetrahedral coordination geometry of the metal ion in presence of N-terminal glutaminyl substrates, different experiments were applied to elucidate the binding mode of N-terminal glutamyl substrates. Cyclization of N-terminal glutamic acids correlates to the pathophysiologic function of hQC, which only occurs at the Aß peptide species after proteolytic processing of the amyloid precursor protein (APP) with $\beta$ and $\gamma$-secretase. After proteolytic cleavage the $A ß$ peptide harbors a glutamic acid at its first N-terminal amino acid position. In kinetic studies it was shown that turnover of pathophysiological substrates was drastically reduced compared to physiological substrates (Fig. 6.7). To investigate if the coordination geometry of N-terminal glutamyl substrates differ from the N-terminal glutaminyl substrates (QFRH), the tetrapeptide EFRH was utilized for all experiments. A deviation of the standard tetrahedral coordination geometry would be able to influence catalysis and might explain the decelerated turnover of N-terminal glutamyl substrates.

To verify this theory stopped flow UV/Vis absorption spectroscopy with Co(II)-hQC in 


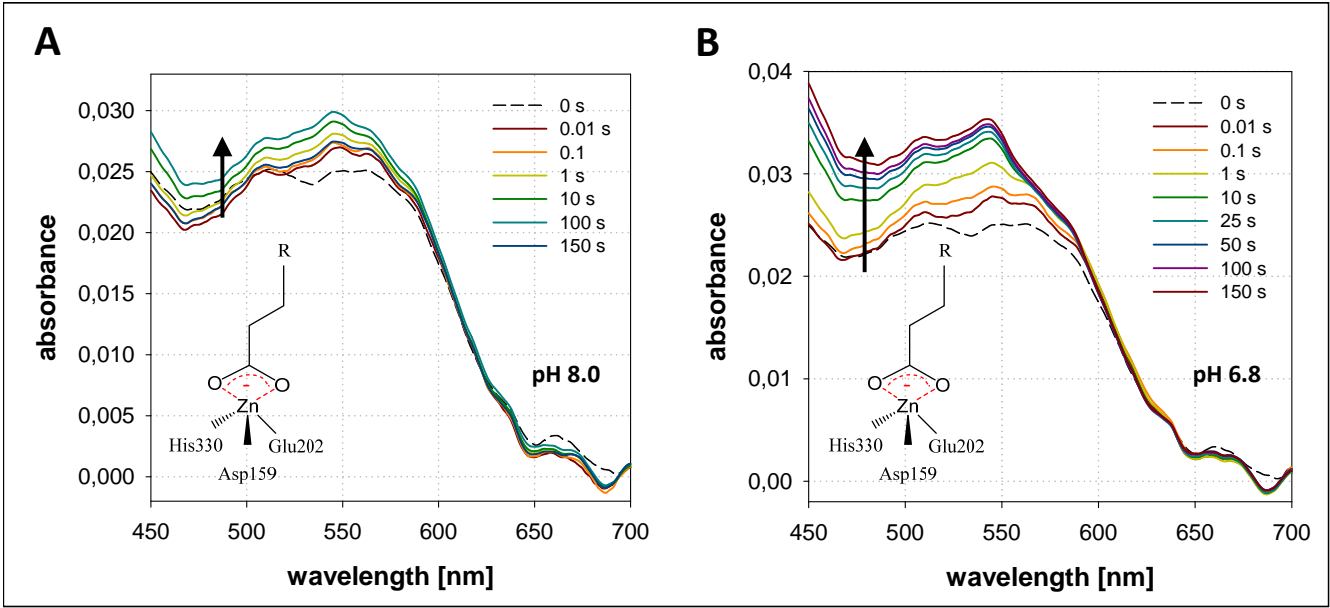

Figure 6.17: Time resolved UV/Vis absorption spectra of Co(II)-hQC after mixing with the tetrapeptide EFRH. For measurements a final concentration of $102 \mu \mathrm{M} \mathrm{Co}(\mathrm{II})$-hQC and $1 \mathrm{mM}$ EFRH after mixing were utilized. Arrows indicating the change of absorption over time. Scheme of the coordination geometry should elucidate how binding of N-terminal glutamyl substrates to the originally zinc might look like. Used buffers were Chelex-100 treated before measurements. Depicted spectra are buffer and protein corrected. (A) Time resolved UV/Vis absorption spectra at alkaline pH conditions. Measurements were performed in $50 \mathrm{mM}$ Tris/ $\mathrm{HCl}, \mathrm{pH} 8.0$, at $10^{\circ} \mathrm{C}$. (B) Time resolved UV/Vis absorption spectra at acidic $\mathrm{pH}$ conditions. Measurements were performed in $30 \mathrm{mM}$ Tris/ $\mathrm{HCl}$ and $20 \mathrm{mM} \mathrm{MES,} \mathrm{pH}$ 6.8 , at $30^{\circ} \mathrm{C}$.

presence of the tetrapeptide EFRH was performed. Kinetic studies of hQC catalyzing the tetrapeptide EFRH utilizing the continuous calorimetric activity assay showed detectable heat release at acidic $\mathrm{pH}$ conditions, but not at alkaline $\mathrm{pH}$ values (Fig. 6.7). Therefore, measurements were performed at acidic $(\mathrm{pH}$ 6.8) and alkaline $(\mathrm{pH}$ 8.0) $\mathrm{pH}$ conditions to investigate if binding of EFRH depends on the protonation state of the substrate, which was shown for N-terminal glutaminyl substrates (Tab. 6.1) [54]. In this case, no change in the absorbance intensity over time at alkaline $\mathrm{pH}$ conditions should be observed. However, stopped flow UV/Vis absorption spectra exhibited a change in absorbance intensities after mixing of $\mathrm{Co}(\mathrm{II})-\mathrm{hQC}$ and EFRH at alkaline $\mathrm{pH}$ conditions (Fig. 6.17). This observation indicates binding and coordination of the N-terminal glutamyl substrate towards the metal ion within the active site, which does not depend on the protonation state of the substrate. Interestingly, obtained time resolved UV/Vis spectra of $\mathrm{Co}(\mathrm{II})-\mathrm{hQC}$ in presence of the tetrapeptide EFRH at both $\mathrm{pH}$ conditions differed from the spectrum of the enzyme in resting state. Due to the fact that the characteristic UV/Vis spectrum of Co(II)-hQC in resting state is associated with a tetrahedral coordination of the central metal ion, it can be assumed that the change in the UV/Vis absorption signal might be an attribute of a switched coordination geometry. Summarizing the obtained information of the EPR 
experiment for N-terminal glutamyl substrates, it can be assumed that the change in the $\mathrm{UV} / \mathrm{Vis}$ absorbance spectra might be assigned to a five-fold coordination of the central metal ion. Interestingly, freeze quench EPR experiments with $\mathrm{Co}(\mathrm{II})$-isoDromeQC already indicated that binding and coordination of an N-terminal glutamic acid differs compared to N-terminal glutamines (Fig. 6.18). It was shown that the EPR signal shifted into the higher magnetic field from $1038 \mathrm{G}$ (resting state) to $1440 \mathrm{G}$ (in complex with EFRH). In addition, the band width of the EPR signal for Co(II)-isoDromeQC in complex with EFRH was broadened significantly compared to the obtained EPR signal for Co(II)-isoDromeQC in resting state and in complex with QFRH or pEFRH. Accordingly, due to the different EPR signal a complete tetrahedral coordinated species can be excluded. Furthermore, characteristics of the EPR signal indicated a five-fold coordinated central metal ion in presence of the tetrapeptide EFRH. This observation implies that the Aß peptide species with its N-terminal glutamic acid coordinates bidentally to the catalytic zinc ion within the active site of hQC.

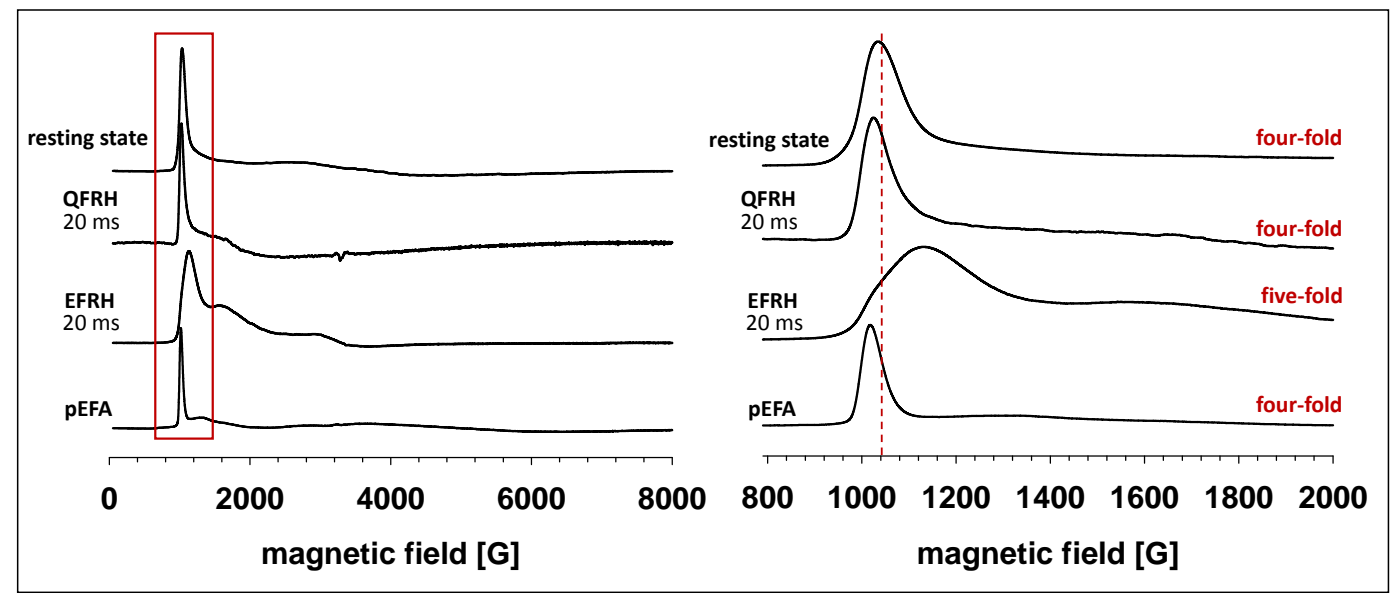

Figure 6.18: EPR spectra of Co(II)-isoDromeQC after incubation with N- terminal glutaminyl-/glutamyl substrates. Entire EPR spectra are depicted on the left side, whereas on the right side an image enlargement of the important region of each spectra is shown. Used ligands with the corresponding incubation time are described, respectively. Measurements were performed at $4.0 \mathrm{~K}$ and $20 \mathrm{~mW}$ at X-band wavelength $(9.36 \mathrm{GHz})$ in $50 \mathrm{mM} \mathrm{NaH}_{2} \mathrm{PO}_{4}, 10 \mathrm{mM} \mathrm{NaCl}, \mathrm{pH}$ 6.8. Molar enzyme concentration was $0.23 \mu \mathrm{M}$ for each measurement. Final concentrations for utilized ligands were $5 \mathrm{mM}$ for QFRH , $15 \mathrm{mM} \mathrm{EFRH} \mathrm{and} 15 \mathrm{mM}$ pEFRH. EPR data were taken from Alexander Piontek.

To gain deeper insights into the bidentally coordinating glutamic acid of EFRH to the catalytic zinc ion within the active site, crystal structures of hQC in complex with the pathophysiological substrate were applied. Crystallization attempts were successful and analyzable data were achieved. 


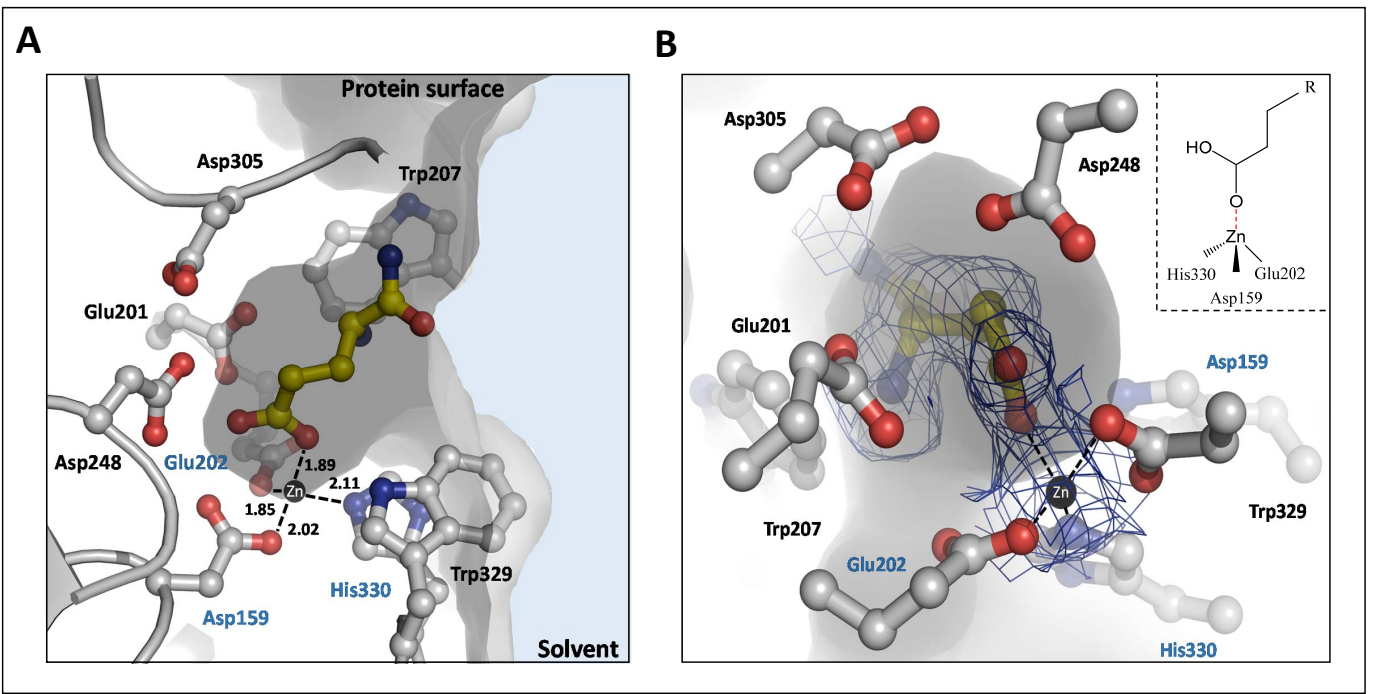

Figure 6.19: Crystal structure of hQC in complex with the tetrapeptide EFRH. (A) Side view of the active site of hQC with coordinated EFRH. Active site residues are shown in grey and named respectively (blue $=$ coordinating residues, black $=$ active site residues), while the substrate is coloured yellow. The hydrophobic pocket is indicated in transparent gray, in which the coordinated zinc (black sphere) is located at the bottom of the pocket. Dashed lines are indicating coordinating bonds with the corresponding distance in $\AA$. (B) Front view of the active site with the corresponding $2 \mathrm{mFo}-\mathrm{DFc}$ electron density map with a sigma contour level of $1.0 \mathrm{\sigma}$. Scheme in the upper right corner should indicate coordination of the metal ion. Resolution $=1.92 \AA$, R-work $=17.9 \%$ and R-free $=20.8 \%$

Crystal structure of hQC in complex with EFRH showed no distinct electron density reflecting a bidental coordination of the N-terminal glutamic acid. Moreover, binding of the substrate towards the metal ion possessed a tetrahedral coordination geometry, in which the coordination bond distances and angles (Tab. 8.1 and Tab. 8.2) showed typical characteristics of a tetrahedron. However, crystal structure of $\mathrm{Co}(\mathrm{II})$-isoDromeQC in complex with EFRH from Alexander Piontek (data not published) showed a remarkably impression of the active site in complex with the tetrapeptide EFRH (Fig. 6.20). Crystal structure of isoDromeQC clearly revealed an alternative conformation of the N-terminal glutamic acid of the EFRH peptide with an occupancy of 0.5, meaning both conformations were up to $50 \%$ present within the crystal. These alternative conformations resulted in a monodental or bidental N-terminally coordinated glutamic acid, leading to a four- or fivefolded coordinated metal ion. Hence, after binding of the N-terminal glutamyl substrate, coordination of the $\gamma$-carbonyl moiety of the side chain seems to be a dynamic process in which either one of the $\gamma$-oxygen atoms form a coordination bond (monodental) or both are coordinated towards the central metal ion (bidental). As already described in literature, coordinating carboxylate ligands have the ability to change their binding mode at metal centers. This process is known as carboxylate shift [109]. The carboxylate shift is able to 
influence reaction kinetics of enzymes due to the switching binding mode of the carboxylate group. In particular, it was reported for the methane monooxygenase to contain a $\mu$ carboxylato bridged binuclear iron site in its active centers, in which the carboxylate shift plays a crucial role in the catalytic pathways of the enzyme [109-111]. For the transition metal zinc a direct observation of the carboxylate shift has only been reported for binuclear model complexes of zinc using NMR spectroscopy [112]. Coordination bond distances and angles are summarized in Tab. 8.1 and Tab. 8.2 respectively, which showed only slight deviations. In conclusion, combined results of EPR, stopped flow UV/Vis absorption spectroscopy and X-ray crystallography for hQC and isoDromeQC, respectively, showed an additional coordination geometry for N-terminal glutamyl substrates, which differs in its coordination number compared to binding of N-terminal glutaminyl substrates. The $\gamma$ carboxylate moiety of the N-terminal glutamic acid could be either monodental or bidental coordinated towards the catalytic zinc ion. Furthermore, a carboxylate shift was observed, which directly influenced the change in the binding mode of the coordinated glutamic acid.
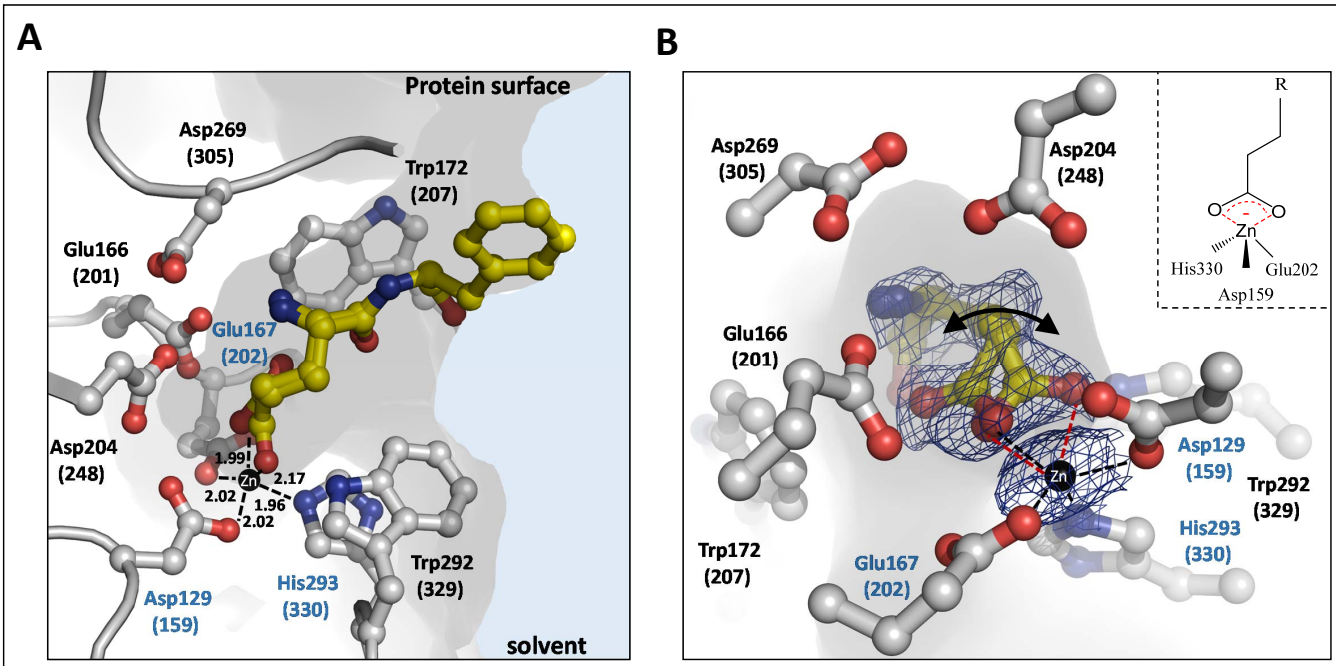

Figure 6.20: Crystal structure of isoDromeQC in complex with the tetrapeptide EFRH. (A) Side view of the active site of $i s o D r o m e Q C$ with coordinated EFRH. Active site residues are shown in grey and named respectively (blue $=$ coordinating residues, black $=$ active site residues), while the substrate is colored yellow. Residue numbers in brackets indicating the corresponding position in hQC. Hydrophobic pocket is indicated in transparent gray, in which the coordinated zinc (black sphere) is located at the bottom of the pocket. Dashed lines are indicating coordinating bonds with the corresponding distance in $\AA$. (B) Front view of the active site with the corresponding $2 \mathrm{mFo}-\mathrm{DF}$ e electron density map with a sigma contour level of $1.0 \sigma$. Scheme in the upper right corner should indicate coordination of the metal ion. Resolution $=1.12 \AA$. Data were taken from Alexander Piontek. 


\subsubsection{Coordination Geometry of the Metal Ion Within the Active Site Depends on Binding of Glutaminyl/Glutamyl Substrates - Role in Catalysis}

Coordination number of the central metal ion of hQC depends on the binding of N-terminal glutaminyl/glutamyl substrates. In case of the physiological substrate the central metal ion has four coordinated ligands, while for the pathophysiological substrate the coordination number switches between four and five coordinated ligands. This distinct change in the coordination geometry caused by a carboxylate shift after binding of an N-terminal glutamyl substrates could explain the difference in their catalytic efficiency compared to N-terminal glutaminyl substrates, which will be discussed in this chapter.

As described previously, catalysis of the physiological substrate within the active site of hQC displayed a pH dependency [54] (Fig. 6.6 and Tab. 6.1). Conversion of glutamine into pyroglutamic acid is favored at alkaline $\mathrm{pH}$ conditions due to the requirement of a deprotonated $\alpha$-amino group, which gets in turn activated for catalysis by protonation of the acid-base catalyst Glu201. After coordination of the substrate towards the central metal ion, the catalytic zinc ion acts as a Lewis acid and extracts electrons from the $\gamma$-oxygen of the N-terminal glutaminyl side chain. Accordingly, electrophilicity of the $\gamma$-carbonyl

carbon atom is drastically increased, resulting in a nucleophilic attack of the $\alpha$-amino group. A prerequisite for the nucleophilic attack is a tetrahedrally coordinated central metal ion and a non-coordinating $\gamma$-amino group, which acts as a leaving group and splits off from the transition intermediate during catalysis. However, in case of the N-terminal glutamyl substrates the $\gamma$-amino group is exchanged against a hydroxyl group compared to N-terminal glutamines, leading to drastic changes of the catalytic pathway. Free glutamic acids in solution have a $\mathrm{p} K_{a}$ value of 4.2 for the $\gamma$-carboxylic moiety and 9.6 for the $\alpha$-amino group. These $\mathrm{p} K_{a}$ values probably differ and do not reflect the real $\mathrm{p} K_{a}$ values after binding of the substrate compared to the experimentally determined $\mathrm{p} K_{a}$ values of free glutamic acids in solution. Nevertheless, the described $\mathrm{p} K_{a}$ values for free glutamic acids in solution should roughly reflect the given situation within the active site of hQC. The reason for deviated $\mathrm{p} K_{a}$ values is probably that the residue environment within the active site directly influences the $\mathrm{p} K_{a}$ values. In theory, at alkaline $\mathrm{pH}$ conditions, in which physiological substrates are preferentially catalyzed, the $\alpha$-amino group and the $\gamma$-carboxylic moiety would be predominantly deprotonated (Fig. 6.21). Deprotonation of 
the $\gamma$-carboxylic moiety would allow a bidental coordination of both $\gamma$-oxygens towards the zinc ion, resulting in a five-folded coordination of the central metal ion. Hence, due to the bidental coordination both $\gamma$-oxygens are charged equivalently, thereby prohibiting electrophilic activation of the $\gamma$-carbonyl carbon. Therefore, a nucleophilic attack by the $\alpha$-amino group on the $\gamma$-carbonyl carbon is very unlikely, due to the missing electrophile. Thus, at neutral pH conditions the $\gamma$-carboxylic moiety should be still deprotonated, leading to a predominantly five-folded coordinated central metal ion. Only at neutral $\mathrm{pH}$ some species of the $\alpha$-amino group are protonated, thereby decreasing slightly nucleophilicity. However, switching to acidic $\mathrm{pH}$ conditions induces a carboxylate shift of the $\gamma$-carboxylic moiety by protonating one of the $\gamma$-oxygens, which results in a tetrahedral coordinated central zinc ion. Additionally, more species of the $\alpha$-amino group are protonated at acidic $\mathrm{pH}$, which decrease nucleophilicity drastically. Concerning reduced turnover of N-terminal glutamyl substrates compared to N-terminal glutaminyl substrates catalyzed by hQC, the $\mathrm{pH}$ dependent carboxylate shift induced by protonation of the $\gamma$-carboxylic moiety, thereby switching coordination geometry from five-fold to four-fold, is probably the major factor for differences in catalysis. Furthermore, only a small number of deprotonated $\alpha$-amino group and protonated $\gamma$-carboxylic moiety species are available at acidic $\mathrm{pH}$ conditions, which in turn also influences turnover of N-terminal glutamic acids. The first model relies on the fact that the proton for protonation of one $\gamma$-oxygen comes from solution, but considering coordination of the substrate towards the central metal ion probably induces a $\mathrm{p} K_{a}$ shift. Coordination of both $\gamma$-oxygens towards the zinc ion could lower the $\mathrm{p} K_{a}$ value for the $\gamma$-carboxylic moiety, because the zinc ion is a strong Lewis acid and pull out electrons from the $\gamma$-carboxylic moiety. A shifted $\mathrm{p} K_{a}$ would affect the protonation of the $\gamma$-carboxylic moiety by the solvent drastically, meaning protonation of the $\gamma$-carboxylic moiety by the solvent is improbable. Therefore a second model was developed (Fig. 6.21), in which protonation of the $\gamma$-carboxylic moiety is induced by a spatially close amino acid residue within the active site of hQC. Crystal structures of hQC revealed Glu201 as a potential proton donor candidate, which could transfer a proton to induce the carboxylate shift. Experimental determined $\mathrm{p} K_{a}$ values of glutaminyl cyclase from Carica papaya catalyzing Glu- $\beta$ NA were 7.5 and 4.2, respectively [3]. Therefore, it might be possible that some $\gamma$-carboxylic moieties of Glu201 get protonated at acidic $\mathrm{pH}$ conditions. The proton is subsequently transferred to the coordinating substrate, thereby inducing the carboxylate 
shift. In summary, two proposed models were developed to postulate the influence of the coordination number of the central zinc ion in terms of catalysis of N-terminal glutamyl substrates.

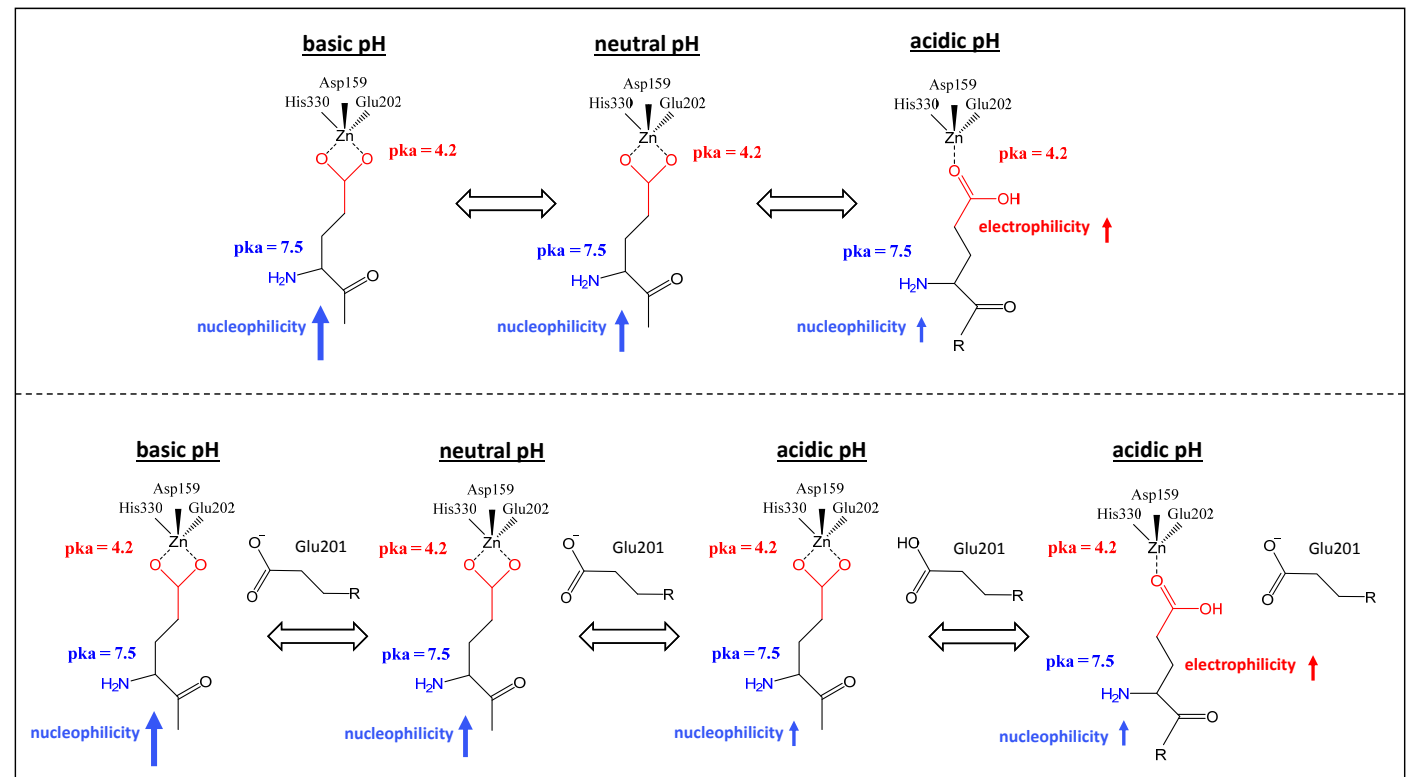

Figure 6.21: Proposed model for an induced carboxylate shift of N-terminal glutamyl substrates coordinated to the catalytic zinc ion of hQC. Upper scheme shows a pH-dependent carboxylate shift of the $\gamma$-carboxylic moiety of the N-terminal glutamic acid in which the proton is donated from solution. Lower scheme represents an induced carboxylate shift by a surrounding amino acid residue transferring a proton onto $\gamma$-carboxylic moiety of the substrate. The $\gamma$-carboxylic moiety (red) and the $\alpha$-amino group (blue) are highlighted, respectively. Arrows indicate strong (thick) or weak (thin) nucleophilicity/electrophilicity of the corresponding functional groups. After the carboxylate shift the Nterminal glutamic acid is monodentally coordinated to the catalytic zinc ion and can take the next step in the catalytic cycle of hQC.

\subsubsection{Summary}

Substitution of zinc against cobalt within the active site resulted in spectroscopically active $\mathrm{Co}(\mathrm{II})$-hQC with comparable activities compared to hQC. The almost equal ionic radii and the Alfred-Rochow electronegativities $(\mathrm{Zn}=1.66$ and $\mathrm{Co}=1.70$ [113]) are probably the reasons for beneficial commutability. Based on the successful substitution of the cobalt different spectroscopic methods, for example stopped flow UV/Vis spectroscopy, were applied to investigate the coordination number of the central metal ion after binding of N-terminal glutaminyl/glutamyl substrates. In addition, crystal structures of hQC in complex with different substrates or the product were generated. Obtained experimental data revealed a four-folded tetrahedral coordinated metal ion in case of N-terminal glutaminyl substrate and the product, whereas for the glutamyl substrates a mixture of 
four- and five-fold coordinated metal ion was present. Furthermore, a pH dependent carboxylate shift was observed for N-terminal glutamyl substrates, inducing a switch from a five-fold coordinated to a four-fold coordinated metal ion. This difference in the coordination geometry of the enzyme-metal complex presumably plays a major role in catalysis of N-terminal glutamyl substrates. A bidental coordination of the substrate would prohibit the cyclization reaction of glutamic acid to pyroglutamic acid due to the lack of an electrophilic $\gamma$-carbon atom, while a monodental coordinated substrate comply all prerequisites for an intramolecular nucleophilic attack of the $\alpha$-amino group onto the $\gamma$-carbon atom.

\subsection{Design and Characterization of a Potent Inhibitor for Human Glutaminyl Cyclase}

Human glutaminyl cyclase is a potential drug target candidate for the development of inhibitors in the treatment of Alzheimer's disease. As described recently, hQC has the ability to convert $\mathrm{N}$-terminal glutamic acids of the $\mathrm{A} ß$ peptide species into the respective cyclized product in an in vitro experiment. This observation supports the idea of a possible involvement of hQC in the initiation of the neurotoxic plaque formation in Alzheimer's disease [3]. The aim of this study was to gain comprehensive information about the catalytic properties, the structure and in particular the coordination geometry of the metal ion within the active site of hQC. Based on these findings, design of a potent inhibitor for hQC mimicking a highly energetic transition intermediate was attempted. Therefore, potential transition state analogue compounds were tested and kinetically characterized via the continuous calorimetric activity assay. The resulting $K_{\mathrm{i}}$ constant served as a scale for comparison among tested compounds. The most promising candidate was further characterized by different methods, like thermodynamic characterization via isothermal titration calorimetry and X-ray crystallography, to elucidate the nature of the enzymeinhibitor complex.

\subsubsection{The First Described Imidazole Based Inhibitor for Human Glutaminyl Cyclase}

Most metalloenzyme inhibitors are drug-like small molecules, consisting of a metal binding group (MBG) and a backbone group. The MBG coordinates to the active site metal ion, whereas the backbone group is specifically customized for their respective targets, which 
Figure 6.22: Chemical structure of the hQC inhibitor PBD150 The inhibitor is subdivided into two important regions, which are connected via a linker. $\mathrm{MBG}=$ metal binding group.



could consists of substituted aliphatic chains, aromatic rings, or heterocycles. The specific customization of the backbone group for an active site of an enzyme should in theory increase specificity of the inhibitor for its target and reduce off-target inhibition. In addition, the MBG and the backbone group are connected via a linker. As already published in former studies, a first effective competitive inhibitor for hQC was described and stepwise optimized based on the general matrix for metalloenzyme inhibitors. As MBG the metal chelator imidazole was chosen. Imidazole tends to interact with accessible zinc atoms and was consequently reported as a weak inhibitor of hQC with an inhibitory constant in the lower micromolar range $\left(K_{\mathrm{i}}=103 \pm 4 \mu \mathrm{M}\right)$ [23]. Imidazole is commonly used as a metal chelator and capable to coordinate zinc ions, which was shown for the active site of carboxypeptidase A [114]. Starting from imidazole as the metal binding group the compound was improved by applying a ligand-based optimization leading finally to the inhibitor 1(3-(1H-imidazol-1-yl)propyl)-3-(3,4-dimethoxyphenyl)thiourea (PBD150) [56] (Fig. 6.22). The linked backbone group is in general a hydrophobic tail customized specifically for the active site of hQC. Accordingly, the designed hydrophobic tail in combination with imidazole improved the inhibitory power drastically, leading to a inhibitory constant in the lower nanomolar range $\left(K_{\mathrm{i}}=60 \pm 0.2 \mathrm{nM}\right)$ [56]. Potency of PBD150 was shown in transgenic mouse models, in which the formation of $\mathrm{A} \beta_{3(G l u)-40 / 42}$ to $\mathrm{A} \beta_{3(p G l u)-40 / 42}$ was drastically reduced, resulting in a significantly decreased deposition of toxic plaques within the brain [115]. These experimental data support the hypothesis of modified amyloid-beta peptides acting as a seeding peptide species in the amyloid cascade, confirming hQC as a valuable drug target in the treatment of Alzheimer's disease. However, homology modeling allowed to get first insights in the theoretical binding mode of PBD150 within the active site of hQC [116], which were confirmed by crystal structures of mammalian QC in complex with PBD150 (Fig. 6.23 A) [117]. Besides the coordination of the imidazole moiety towards the zinc ion, formation of a hydrogen bond established directly between the thio-carbonly 
group and the backbone amide of Gln304 was observed (Fig. 6.23 B). The dimethoxyphenol moiety rests upon a hydrophobic patch on the surface of the enzyme, while the two methoxy groups are involved in solvent-mediated hydrogen bonds to the backbone amide of Phe325. Nevertheless, imidazole is known to be a common metal chelator and could in theory cause severe side effects within the human body by targeting accidentally different metal dependent enzymes. Previous publications showed that off-target inhibition of inhibitors even with a specifically customized backbone is possible [118]. Another point of interest was to find a non-imidazole based MBG, which binds stronger to the catalytic zinc ion and might thereby increase inhibitory power and specificity. Consequently, for development of a highly potent competitive non-imidazole based inhibitor for hQC, screening for an alternative and stronger coordinating metal binding group specifically targeting the zinc ion had highest priority.

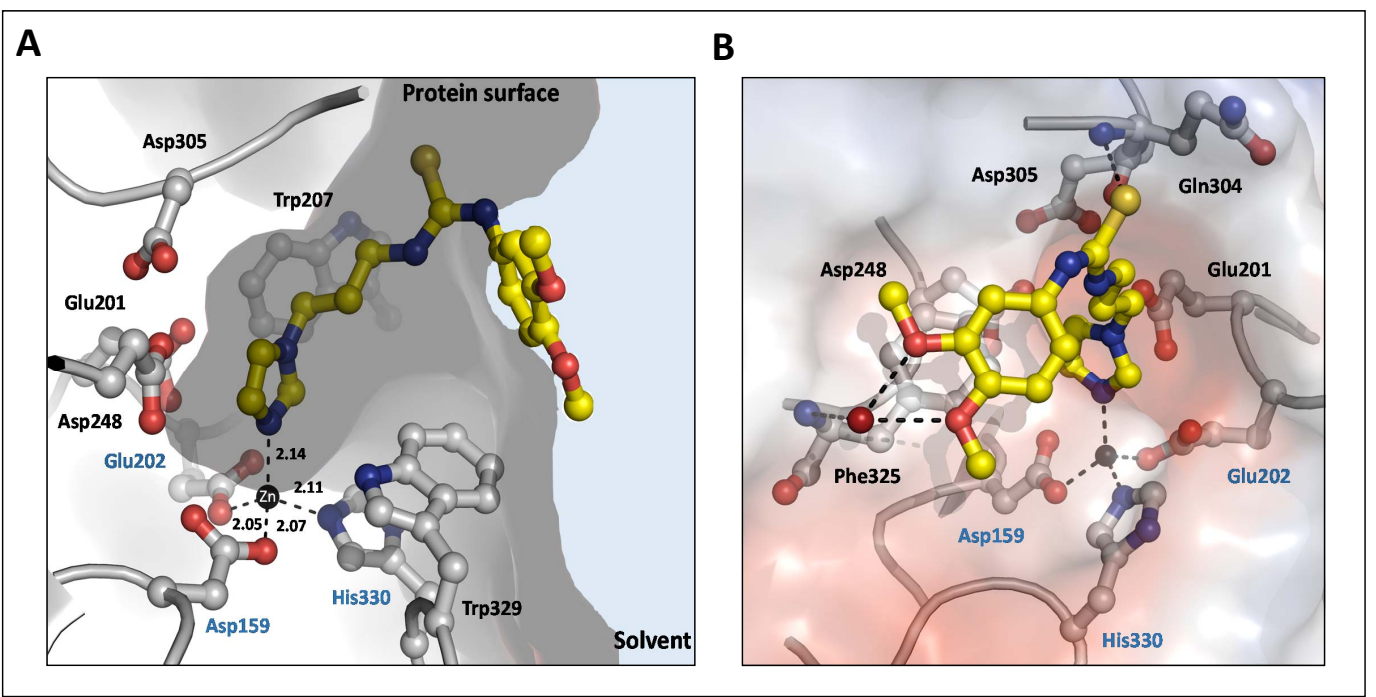

Figure 6.23: Crystal structure of human glutaminyl cyclase in complex with the inhibitor PBD150. (A) Side view of the active site of hQC with coordinated PBD150. Active site residues are shown in grey and named respectively. Blue colored residue names represent zinc coordinating residues, while black stands for important active site residues involved in catalysis or substrate binding. The coordinated inhibitor is colored yellow. Hydrophobic cavity is indicated in transparent gray, in which the coordinated zinc (black sphere) is located at the bottom of the pocket. Dashed lines indicate coordinating bonds with the corresponding distance in $\AA$. (B) Back view of the inhibitor PBD150. Electrostatic potentials of the protein surface are reflecting negatively (red) and positively (blue) charged regions. Interactions with additional residues are indicated by dashed lines. Presented structures were modeled from PBD entry 3PBB. Resolution $=1.95 \AA$ 


\subsubsection{Screening for a New Metal Binding Group for Glutaminyl Cyclase Revealed a Carbohydrazide Function as a Potential Candidate}

One strategy in the treatment of Alzheimer's disease is the inhibition of glutaminyl cyclase in order to avoid harmful accumulation of highly amyloidogenic species in brain tissues. For this purpose, screening for a new highly specific metal binding head group for hQC, which should have a stronger affinity compared to the imidazole moiety of PBD150, was performed. To prevent artificial binding of the metal binding group to metalloenzymes in general, different points were considered in order to increase specificity. Mechanical studies revealed that the catalytic zinc ion of hQC is not sterically restricted to adopt a five-folded coordination geometry, which was shown for the crystal structure of isoDromeQC in complex with the tetrapeptide EFRH (see chapter 6.4.7). Therefore, screening for a metal binding group coordinating bidentally to the catalytic zinc ion could increase affinity and specificity towards the enzyme. Additionally, compounds were selected mimicking transition state intermediates of the hQC cyclization reaction to enhance specificity.

To first verify if binding our chosen potential compounds is feasible, a first kinetic characterization by utilizing the continuous calorimetric activity assay for each single specifically selected compound was performed. By applying the new developed calorimetric activity assay (see chapter 6.2.2), the obtained experimental data was used to determine the inhibitory constant $\left(K_{\mathrm{i}}\right)$ according to Dixon [70]. The determined $K_{\mathrm{i}}$ value was used as a scale for comparison of inhibitory power among tested compounds. For a typical $K_{\mathrm{i}}$ determination defined compound concentrations were mixed with the standard substrate Gln-Gln (QQ) and loaded in the measurement cell of the ITC device followed by an injection of the enzyme to trigger the reaction. Calorimetric progress curves of hQC inhibited by a potential compound should in theory differ from progress curves in absence of the potential compound. In general, complete depletion of the substrate should take longer in presence of a competitive inhibitor due to the fact that substrate and inhibitor compete for the binding site, leading to an apparent Michaelis-Menten constant $\left(K_{\mathrm{M}}\right)$ for the substrate. 
Table 6.3: Overview of tested inhibitors for hQC. For determination of the inhibitory constants $K_{\mathrm{i}}$ the continuous calorimetric activity assay (see chapter 6.2.2) was utilized. Measurements were performed in $50 \mathrm{mM}$ Tris $/ \mathrm{HCl}, \mathrm{pH} 8.0$, at $30{ }^{\circ} \mathrm{C}$.

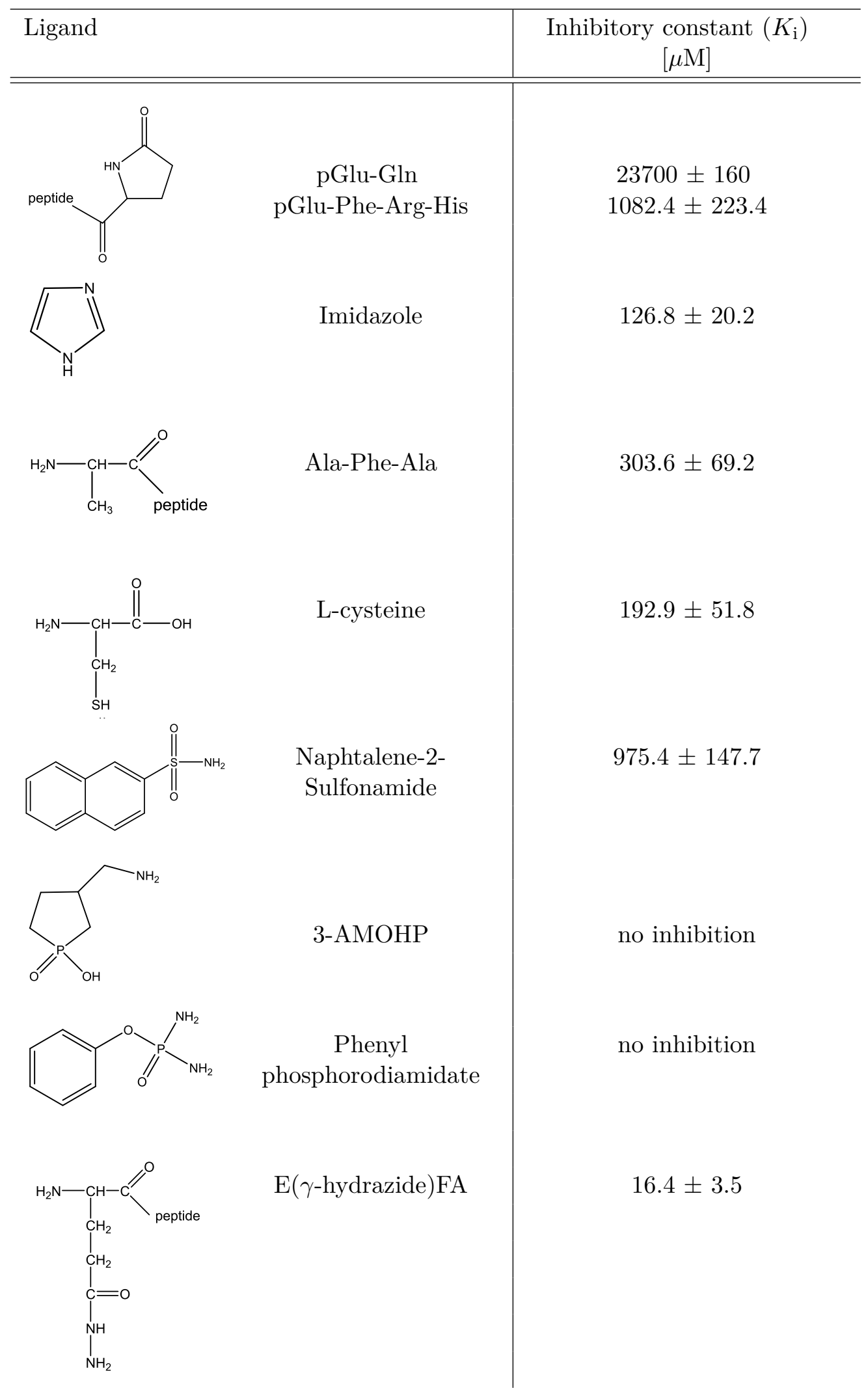


Zinc is classified as a class A acceptor, which forms stronger complexes with the firstrow donor atoms oxygen or nitrogen than with second-row elements sulfur or phosphorus [119]. Accordingly, compounds were selected which theoretically coordinate via oxygen or nitrogen to the zinc ion of $\mathrm{hQC}$ and in addition contain a hydrophobic patch as the backbone group, like phenyl phosphorodiamidate or 3-(guanido)-1-oxo- 1-hydroxy-phospholane (3-AMOHP) for instance. Furthermore, the amino acid cysteine, which could in theory coordinates via its thiol side chain to the catalytic zinc ion, was also tested. Cysteines can display high affinity toward zinc ions, resulting in a metal complex formation. It was demonstrated that metal ions coordinated by cysteine residues can be a critical mediator of protein structure, catalysis and regulation [120]. Experimentally determined $K_{\mathrm{i}}$ constants for tested compounds are summarized in Tab. 6.3. First, imidazole was tested to check if utilizing the calorimetric activity assay results in comparable $K_{\mathrm{i}}$ values between the experimentally determined and the published $K_{\mathrm{i}}$ value. Imidazole showed a nearly identical $K_{\mathrm{i}}$ value compared to the published one [23], verifying kinetic characterization of inhibitors by calorimetry as a suitable tool. Unfortunately, no inhibition of hQC activity could be observed in case of the compounds phenyl phosphorodiamidate (commercially available) and 3-AMOHP (synthesized by Brigitte Worbs in the Department of Organic and Biomolecular Chemistry, AK Diederichsen). One explanation might be that both compounds were sterically impeded to be able to coordinate to the catalytic zinc ion in the active site of hQC. This was especially surprising for the compound 3-AMOHP, due to the fact that it should mimic the tetrahedral transition intermediate during catalysis of N-terminal glutaminyl/glutamyl substrates. In addition, cyclic phosphinic acids were described to be common inhibitors, for instance by binding to $\gamma$-aminobutyric acid (GABA) receptors [121] However, the compound naphtalene-2-sulfonamide (commercially available) exhibited a weak inhibitory effect on pyroglutamyl formation catalyzed by hQC in the higher micromolar range. Sulfonamide was reported to inhibit competitively enzymes in the pathway of folic acid synthesis [122]. Nevertheless, sulfonamide was excluded as potential MBG due to their $\sim 7$-fold decreased binding affinity compared to imidazole. As expected, the amino acid L-cysteine showed an inhibitory effect on the hQC activity, probably by occupying the fourth coordination site of the catalytic zinc via its thiol side chain. However, thiol groups as potential MBG coordinating to the zinc ion in the active site of hQC were also excluded due to their $\sim 2$-fold increased $K_{\mathrm{i}}$ value. Interestingly, the 
tripeptide Ala-Phe-Ala (AFA) showed an inhibitory effect with a $K_{\mathrm{i}}$ value in the middle micromolar range $\left(K_{\mathrm{i}}=303.5 \pm 69.2 \mu \mathrm{M}\right)$. An explanation was given by the crystal structure of isoDromeQC in complex with the tripeptide [99]. The bound tripeptide adopted a conformation within the active site spatially close to the catalytic metal ion resulting in two hydrogen bond formations. First, the $\alpha$-nitrogen of the phenylalanine in the second position of the tripeptide forms a hydrogen bond with the oxygen of the main chain of Glu292 and, secondly, the $\alpha$-nitrogen of the main chain of Glu292 interacts with the carbonyl oxygen of phenylalanine of the tripeptide. Due to the conformation of the bound tripeptide and further lack of a potential metal binding group the catalytic zinc ion was still coordinated tetrahedrally, in which the fourth coordination site was occupied by the water molecule.

Interestingly, a new potent metal binding group for hQC was discovered by substituting the $\gamma$-oxygen of a glutamic acid against a hydrazide function $(\mathrm{E}(\gamma$-hydrazide $)) . \mathrm{E}(\gamma$-hydrazide $)$ was first described to serve as a potent inhibitor of glutamine metabolism in liver mitochondria [123]. Besides the metal binding group a backbone group was introduced consisting of phenylalanine and alanine ( $\mathrm{E}(\gamma$-hydrazide $) \mathrm{FA})$. As described previously, bulky and hydrophobic residues at the second and third N-terminal amino acid position, especially phenylalanine, increased substrate affinity (see chapter 6.3.2) (Fig. 6.24 A). Kinetic characterization via the continuous calorimetric activity assay showed a concentration dependent change in the calorimetric progress curves, in which the time of complete substrate depletion was accelerated with increasing $\mathrm{E}(\gamma$-hydrazide)FA concentration (Fig. 6.24 B). Analysis of the experimental ITC data according to Michaelis-Menten kinetics by plotting the turnover number of the $\mathrm{hQC}$ reaction against the substrate concentration in dependency of various $\mathrm{E}$ ( $\gamma$-hydrazide)FA concentrations revealed a typical competitive inhibition mode (Fig. 6.24 C). Competitive inhibition apparently increases the $K_{\mathrm{M}}$ constant, while $V_{\max }$ remains the same, because the presence of the inhibitor can be negotiated by higher substrate concentrations. This effect could be observed for hQC catalyzing the dipeptide Gln-Gln in presence of different $\mathrm{E}(\gamma$-hydrazide)FA concentrations. For determination of the $K_{\mathrm{i}}$ constant according to Dixon [70] the turnover number of various substrate concentrations were plotted reciprocally against the $\mathrm{E}(\gamma$-hydrazide)FA concentration (Fig. 6.24 D). Obtained data points were fitted linear and intersection of linear regressions reflected the $K_{\mathrm{i}}$ value of $\mathrm{E}\left(\gamma\right.$-hydrazide)FA for hQC, which was in the low micromolar range $\left(K_{\mathrm{i}}\right.$ 
$=16.4 \pm 3.5 \mu \mathrm{M})$. In addition, experimental data was also analyzed by three-dimensional plotting and the obtained $K_{\mathrm{i}}$ value was identical $\left(K_{\mathrm{i}}=11.6 \pm 2.3 \mu \mathrm{M}\right)$ (Fig. 8.13). Thus, the inhibitor with its metal binding group $\mathrm{E}$ ( $\gamma$-hydrazide) has a 8 -fold decreased $K_{\mathrm{i}}$ value compared to the metal chelator imidazole. In summary, a potential metal binding group with a stronger affinity for the catalytic zinc ion of hQC compared to imidazole was analyzed and proved to be beneficial.

A<smiles>CC(NC(=O)C1Cc2ccccc2C(=O)NC(=O)C(N)CCC(=O)NN1)C(=O)O</smiles>

C



B

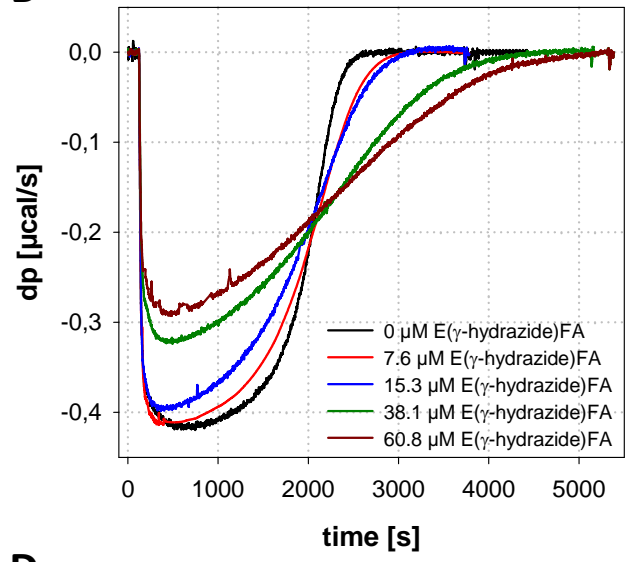

D



Figure 6.24: Kinetic characterization of hQC in presence of the inhibitor $\mathbf{E}$ ( $\gamma$-hydrazide)FA. (A) Chemical structure of the inhibitor $\mathrm{E}(\gamma$-hydrazide)FA. Coordinating metal binding group (MBG) is highlighted in blue, linker in black and backbone group in red. (B) Calorimetric progress curves of hQC activity catalyzing the dipeptide Gln-Gln (QQ) in presence of different $\mathrm{E}(\gamma$-hydrazide)FA concentrations. (C) Michaelis-Menten plot of conversion of the N-terminal glutaminyl substrate catalyzed by hQC at different inhibitor concentrations. Obtained data points were fitted according to Eq. 3. (D) Dixon plot for the inhibition of hQC catalyzed cyclization reaction by $\mathrm{E}(\gamma$-hydrazide)FA. Turnover number at defined substrate concentrations measured for each inhibitor concentration was plotted reciprocally against $\mathrm{E}(\gamma$ hydrazide)FA concentrations. Obtained data points were fitted to a linear dependency and the $K_{\mathrm{i}}$ value was determined by calculating the intersections for each linear regression. 


\subsubsection{X-Ray Structure of the Potential Carbohydrazide Inhibitor Bound by Human}

\section{Glutaminyl Cyclase Revealed a Five-Fold Coordination}



Figure 6.25: Crystal structure of hQC in complex with the inhibitor E( $\gamma$-hydrazide)FA. (A) Left structure shows the inhibitor $\mathrm{E}(\gamma$-hydrazide $) \mathrm{FA}$ with atom nomenclature according to Coot. The right structure shows a simulated mFo-Fc density for the inhibitor $\mathrm{E}(\gamma$-hydrazide $) \mathrm{FA}$ bound by hQC represented with a contour level of $3.0 \sigma$. (B) Side view of hQC active site in complex with E( $\gamma$-hydrazide)FA (yellow). Metal coordinating (blue) and active side (black) residues are named respectively. Coordinating bonds with the zinc (black sphere) are indicated by black dashed lines, while potential hydrogen bonds are represented in blue dashed lines with the corresponding bond length distance in $\AA$. The red sphere indicates a water molecule, which is involved in a solved mediated hydrogen bond. (C) Front view into the active site cavity of hQC in complex with the inhibitor. Electrostatic potential of the protein surface is indicated by red (negatively charged) and blue (positively charged) regions. (D) Observed movement of Trp207 within the active site of the enzyme in absence (I.) and presence (II.) of the inhibitor. Red arrow indicates movement of the amino acid residue. Resolution $=1.95 \AA$, $\mathrm{R}$-work $=17.1 \%$ and $\mathrm{R}$-free $=20.2 \%$

Kinetic analysis of hQC in presence of $\mathrm{E}(\gamma$-hydrazide)FA indicated that the compound functions as a competitive inhibitor. To obtain information about the binding mode of $\mathrm{E}(\gamma$-hydrazide)FA within the active site of hQC, the crystal structure of hQC in complex with the competitive inhibitor was solved. Data collection and refinement statistics are 
summarized in Tab. 8.3.

Analysis of protein crystals revealed complete electron density for all residues of the inhibitor (Fig. 6.25 A). In this study, a defined electron density for all residues of a ligand in complex with the enzyme was established for the first time, indicating a tight binding of the inhibitor to the protein. In addition, the active site of both molecules within the asymmetric unit were fully occupied with $\mathrm{E}(\gamma$-hydrazide)FA (occupancy $=1.0)$. Interestingly, the crystal structure showed that the metal binding group of $\mathrm{E}$ ( $\gamma$-hydrazide)FA coordinates bidentally to the catalytic zinc ion (Fig. $6.25 \mathrm{~B}$ ). The carbonyl $\gamma$-oxygen atom (OE1) and the $\gamma^{\prime}$-nitrogen atom (NE2) of the carbohydrazide function formed a coordinating bond with the catalytic zinc ion, respectively. As a result of this bidental coordination of the MBG, the central metal ion was five-fold coordinated. Bond length and angle for each coordinated ligand are summarized in Tab. 8.1 and Tab. 8.2, respectively. Coordination bond lengths between the catalytic zinc ion and the $\gamma$-oxygen atom $(2.34 \AA)$ or the $\gamma^{\prime}$-nitrogen atom $(2.19 \AA)$ were slightly elongated. A standard coordinated oxygen-zinc bond length should be $2.20 \pm 0.13 \AA$, while in case of nitrogen-zinc it should be $2.09 \pm$ $0.14 \AA[108]$ ). Searching for carbohydrazide functions coordinated to a single zinc ion in the Cambridge Crystallographic Database (CSD) revealed bond lengths in the same range, thereby verifying observed coordination bond lengths in the crystal structure between the catalytic zinc ion and the $\gamma$-oxygen and $\gamma^{\prime}$-nitrogen atom. Interestingly, bond angles of the central catalytic zinc ion and its five coordinating ligands did not show perfect bond angles for a trigonal bipyramidal $\left(\alpha=90^{\circ}, \beta=120^{\circ}\right.$ and $\left.\gamma=180^{\circ}\right)$ or square pyramidal $\left(\alpha=\beta=\gamma=90^{\circ}\right)$ coordination. This observation might indicate an isomerization of the metal complex commonly referred as Berry pseudorotation. A Berry pseudorotation takes place in molecules with a trigonal bipyramidal coordination, in which the central atom has a coordination number of five [124]. The pseudorotation function in a well-defined mechanism, in which atoms perform oscillations in dependency of their local temperature, causing periodic changes of bond lengths and angles within a given metal complex. As a result of this oscillations the metal complex adopts either a trigonal bipyramidal or a square pyramidal geometry, which are converging fluently into each other. For a pseudorotation the two ligands in the apical position of the trigonal bipyramidal geometry should move towards each other, which is in case of hQC the imidazole moiety of His330 and the 
amino group of the carbohydrazide function of $\mathrm{E}(\gamma$-hydrazide) (Fig. 6.26). Due to this movement, the bond angle is reduced from $180^{\circ}$ to $120^{\circ}\left(\right.$ His330-Zn-NE2' $\left.=156^{\circ}\right)$. In addition, two equatorial atoms need to move apart and the bond angle is expanded from $120^{\circ}$ to $180^{\circ}\left(\right.$ Glu202-Zn-OE2' $\left.=146^{\circ}\right)$. During the change of positions a transition state is reached, in which the four moving atoms form the base of a square pyramid, whereas the fixed ligand (Asp159) serves as the pinnacle of the pyramid. At the end of a Berry pseudorotation the complex moves back to its initial state in the form of a trigonal bipyramid.



Figure 6.26: Scheme of the Berry pseudorotation of the coordination center within the active site of hQC. The central zinc ion has a coordination number of five and forms a complex with the metal coordinating residues (grey) and $\mathrm{E}(\gamma$-hydrazide) (yellow). First sphere metal coordinating ligands are numbered and the directions of movement is indicated with arrows, respectively. Starting from a trigonal bipyramidal geometry the apical atoms 1 and 2 move closer to each other, while atoms 4 and 5 move away from each other, but still remain in a equatorial position. Atom number 3 acts as fixed point. As a result the metal complex adopts a square pyramidal geometry. Shape of both geometries are indicated by blue lines. A Berry pseudorotation is a highly dynamic process, in which both geometries converge fluently into each other.

To evaluate which coordination geometry of the zinc complex predominantly existed in the obtained crystal structure the structural parameter for five-fold coordinated compounds $\tau_{5}$ was calculated [125]. The $\tau_{5}$ parameter is in a range of zero to one that gives a tendency which coordination geometry of the ligand coordinating center in a metal complex is present. If $\tau_{5}$ is close to one the coordination geometry is similar to trigonal bipyramidal, while if $\tau_{5}$ is close to zero the coordination geometry is similar to square pyramidal. Calculation of $\tau_{5}$ for hQC in complex with $\mathrm{E}(\gamma$-hydrazide $) \mathrm{FA}\left(\tau_{5}=0.16\right)$ revealed a higher 
tendency for a square pyramidal geometry.

Besides bidental coordination of the carbohydrazide function to the catalytic zinc ion additional inhibitor-enzyme interactions within the active site of hQC could be observed (Fig. $6.25 \mathrm{~B}$ and $\mathrm{C}$ ). In case of the metal binding group of $\mathrm{E}(\gamma$-hydrazide)FA four potential hydrogen bonds could be identified. The first hydrogen bond was formed between the $\gamma$-nitrogen of the carbohydrazide function of $\mathrm{E}(\gamma$-hydrazide) and the $\gamma$-carboxylic moiety of Asp248 and, secondly, between the $\alpha$-amino group of $\mathrm{E}(\gamma$-hydrazide $)$ and the $\gamma$ carboxylic moiety of Glu201. At least, one potential hydrogen bond was formed between the $\gamma^{\prime}$-nitrogen of the carbohydrazide function and the $\gamma$-carboxylic moiety of Asp159, an another one between the $\gamma$-oxygen and the indole moiety of Trp329. These potential hydrogen bonds between the metal binding group of the inhibitor and the surrounding active site residues increase affinity of $\mathrm{E}(\gamma$-hydrazide $) \mathrm{FA}$ for hQC, but could also cause a rigidity of the metal complex. Beside a possible Berry pseudorotation it might be possible that these additional interactions could cause a fixation of the metal binding group. Thus, observed deformation of the bond angles could be provoked by the hydrogen bonds and would not allow movement of the carbohydrazide function, which is a prerequisite for a pseudorotation. Accordingly, deformed bond angles of the catalytic zinc-inhibitor complex could be caused by a Berry pseudorotation or due to a fixation of the metal binding group by additional hydrogen bond formations.

In case of the backbone group a total of three additional hydrogen bond formations between the inhibitor and surrounding residues of the active site were observed in the crystal structure of hQC in complex with $\mathrm{E}(\gamma$-hydrazide)FA. First, a potential hydrogen bond was identified between the $\alpha$-amino group of the phenylalanine of $\mathrm{E}(\gamma$-hydrazide $) \mathrm{FA}$ and the C-terminal carbonyl oxygen of Gln304 and the second one between the C-terminal carbonyl oxygen of the phenylalanine of $\mathrm{E}$ ( $\gamma$-hydrazide)FA and the $\alpha$-amino group of Gln304. At least, formation of a potential solvent mediated hydrogen bond between the carbonyl amino group of the alanine of $\mathrm{E}(\gamma$-hydrazide $) \mathrm{FA}$ and the C-terminal carbonyl oxygen of Val302 was observed. These additional interactions might increase binding strength of the inhibitor to hQC and consequently contribute to a stabilizing effect of $\mathrm{E}$ ( $\gamma$-hydrazide)FA binding within the active site. Average B-factor of $\mathrm{E}$ ( $\gamma$-hydrazide)FA was 21.6/27.1 $\AA^{2}$ 
(chain A/chain B), thereby supporting the idea of a stabilized and rigid coordinated inhibitor caused by the tightly bound metal binding group and additional interactions by the backbone group with active site residues. The B-factor (atomic displacement parameter) is commonly seen as a measure to describe the probable position of an atom or molecule in the structure model [126]. A low B-factor represents a part of the structure that is well ordered, whereas a large B-factor generally belongs to a part of the structure that is very flexible [126].

However, overall structure alignment of hQC in resting state and in complex with $\mathrm{E}(\gamma$ hydrazide)FA revealed no large loop movement caused by binding of the inhibitor. Though, Trp207 showed only in one of both independent hQC molecules in the asymmetric unit an alternative conformation (Fig. 6.25 D). The indole ring of Trp207 was in one hQC molecule directed towards the protein surface of the molecule, while in its alternative conformation it was oriented to the opposite direction. Kinetic characterization of site-specific variants by substituting Trp207 against aliphatic residues (e.g. leucine) decreased catalytic efficiency by a factor of 50 [20]. Accordingly, it was postulated that Trp207, which is directly located at the cavity entrance of hQC, plays a role in active site accessibility by adopting two alternative conformations resulting in an open/closed state. However, crystal structure of hQC in complex with $\mathrm{E}(\gamma$-hydrazide)FA showed that the active site of both independent molecules in the asymmetric unit were occupied with the inhibitor. Due to the fact that Trp207 adopted different conformations in the crystal structure, in which one conformation might represented the open state while the other conformation represents the closed state. If this would be the case, one molecule in the asymmetric unit should not have $\mathrm{E}(\gamma$-hydrazide)FA in its active site. Since $\mathrm{E}(\gamma$-hydrazide)FA is present in the active site of both independent hQC molecules, the role of Trp207 during catalysis needs to be reconsidered.

In summary, crystal structure of hQC in complex with $\mathrm{E}(\gamma$-hydrazide)FA enabled a detailed look into the binding mode of the inhibitor. The central catalytic zinc ion with a coordination number of five adopts a predominantly square pyramidal coordination geometry. Furthermore, tight binding and rigidity of $\mathrm{E}(\gamma$-hydrazide $) \mathrm{FA}$ is guaranteed by several additional hydrogen bonds. 


\subsubsection{Binding of the Potential Inhibitor by Human Glutaminyl Cyclase is an Entropically Driven Process}

To investigate thermodynamics of binding of $\mathrm{E}(\gamma$-hydrazide $) \mathrm{FA}$ to hQC, binding studies utilizing isothermal titration calorimetry (ITC) were performed. Characterizing $\mathrm{E}(\gamma$ hydrazide)FA thermodynamically should elucidate the thermodynamic signature and thereby clarify if the binding process is enthalpically or entropically favored. Thermodynamic characterization of binding is in general contributed to hydrophobic interactions and hydrogen bond formation. The principle of hydrophobic interactions is that nonpolar groups associate in a polar environment, leading to minimization of solvent interactions. Ligand binding by hydrophobic interactions is characterized by the release of highly-ordered solvent molecules to bulk solvent, which is slightly endothermic but entropically favored due to a decrease of the organization state. Accordingly, hydrophobic interactions in thermodynamics are characterized by a small positive $\Delta \mathrm{H}$ and a bigger positive $\Delta \mathrm{S}$. In contrast, electrostatic interactions like hydrogen bonds, which are important for biological interactions, are enthalpically favored. However, a generalization is not possible, due to diverse hydrogen bond donor and acceptor properties in free and complex form. In addition, the local environment is also important. Nevertheless, binding strength of inhibitor-target interaction relative to those with solvent, primarily due to hydrogen bond formation and van der Waals interactions is reflected by $\Delta \mathrm{H}$. If $\Delta \mathrm{H}$ is negative, binding is enthalpically favored.

Unfortunately, a stepwise titration of $\mathrm{E}(\gamma$-hydrazide $)$ FA to hQC until the enzyme is completely saturated measured via isothermal titration calorimetry did not result in reliable data (Fig. 8.12). The reason for this is that the Wiseman parameter (c) [127] for hQC binding $\mathrm{E}(\gamma$-hydrazide)FA is below 10 defining the binding process as a "low affinity system". For a "low affinity system" the $c$ value is $<10$ and the shape of an isothermal titration curve is hyperbolic, whereas for $c$ values $>10$ the curve is clearly sigmoidal. Due to the fact that in the first injection all injected ligands should bind to free macromolecules the detected heat release/consumption directly corresponds to $\Delta \mathrm{H}$. Thus, obtaining a reliable $\Delta \mathrm{H}$ value from a "low affinity" binding curve is highly erroneous, because the first part of the sigmoidal curve is missing. As an alternative, the inhibitor was titrated in excess in a single step to the enzyme to ensure complete saturation of hQC (Fig. 6.27 A). 
The integrated heat release of the single titration step is directly proportional to the $\Delta \mathrm{H}$ value. Titration of $\mathrm{E}(\gamma$-hydrazide $) \mathrm{FA}$ to hQC resulted in $\Delta \mathrm{H}^{\circ}=-4.81 \pm 0.25 \mathrm{~kJ} / \mathrm{mol}$, revealing binding of the inhibitor to the enzyme as an exothermic process. As a control, two additional titration steps were performed to exclude artificial binding effects, like interactions between the inhibitor molecules, and to ensure complete saturation of hQC with $\mathrm{E}(\gamma$-hydrazide)FA. The following two titrations are identical and reflecting only heat of dilution of $\mathrm{E}(\gamma$-hydrazide)FA after titration to hQC. At least, to receive a full thermodynamic signature (Fig. 6.27 B) the Gibbs free energy $(\Delta \mathrm{G})$ and the entropy $(\Delta \mathrm{S})$ were calculated according to Eq. 17 . The Gibbs free energy was negative $\left(\Delta G^{\circ}=-27.02 \pm\right.$ $0.31 \mathrm{~kJ} / \mathrm{mol})$, thereby identifying binding of $\mathrm{E}(\gamma$-hydrazide $) \mathrm{FA}$ as a spontaneous exergonic reaction. Interestingly, the entropy was highly positive $\left(\mathrm{T} \Delta \mathrm{S}^{\circ}=-22.28 \pm 0.17 \mathrm{~kJ} / \mathrm{mol}\right)$, meaning that binding of the inhibitor to the enzyme is strongly entropically driven. A possible explanation is that solvent molecules at binding surfaces are higher ordered than bulk solvent and that these molecules can be released or retained by substrate binding. Release of the solvent molecules is entropically favored due to higher disorder, but enthalpically unfavored because of weaker interaction with the bulk solvent. In case of hQC in resting state the active site harbors six water molecules within its cavity plus the interacting water molecules of the unbound inhibitor in solution. These water molecules need to be released for interaction of the inhibitor with the enzyme. A prominent example is the coordinating water occupying the fourth coordination site of the zinc ion, which needs to be displaced and released to bulk solvent for binding of the metal binding group. In conclusion, thermodynamic characterization revealed a entropically driven binding process of $\mathrm{E}(\gamma$-hydrazide)FA to hQC.

\subsubsection{Summary - Future Aspects - Combining Backbone Group of PBD150 with the Carbohydrazide Function}

Screening for a potent non-imidazole based new metal binding group for inhibition of hQC was successful by introducing a carbohydrazide function within the side chain of a glutamic acid. Crystal structure of hQC in complex with the inhibitor showed that $\mathrm{E}(\gamma$-hydrazide) coordinates bidentally to the catalytic zinc ion (Fig. 6.25). As a consequence, the coordination number of the central metal ion increased from four to five. Furthermore, kinetic characterization of hQC in presence of $\mathrm{E}\left(\gamma\right.$-hydrazide)FA exhibited a 8 -fold decreased $K_{\mathrm{i}}$ 


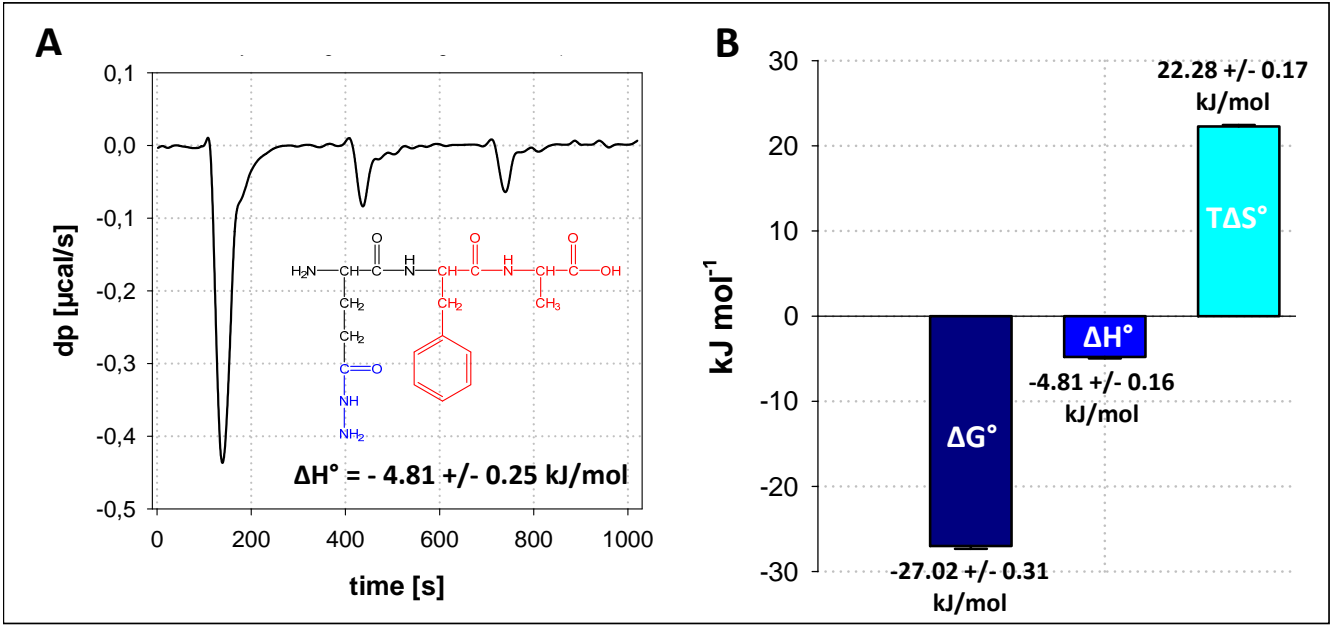

Figure 6.27: Thermodynamic binding studies of $\mathrm{E}(\gamma$-hydrazide)FA binding to hQC. (A) Representative ITC data of a single titration of $\mathrm{E}(\gamma$-hydrazide $) \mathrm{FA}$ to hQC for $\Delta \mathrm{H}$ determination. First peak represents the binding event of $\mathrm{E}(\gamma$-hydrazide)FA to the enzyme, while the last two peaks are only heat of dilution, indicating saturation of hQC with $\mathrm{E}(\gamma$-hydrazide $) \mathrm{FA}$. Measurements were performed in 50 $\mathrm{mM}$ Tris/HCl, $\mathrm{pH} 8.0$, with $0.08 \mathrm{mM}$ hQC in the sample cell and $25 \mathrm{mM} \mathrm{E}(\gamma$-hydrazide)FA in the syrringe. (B) Thermodynamic signature of binding of $\mathrm{E}(\gamma$-hydrazide $) \mathrm{FA}$ to hQC. Whereas $\Delta \mathrm{H}^{\circ}$ was experimentally determined, $\mathrm{T} \Delta \mathrm{S}^{\circ}$ and $\Delta \mathrm{G}^{\circ}$ were calculated according to Eq. 17.

constant compared to imidazole (Fig. 6.3), identifying it as the stronger metal binding group. Nevertheless, the imidazole-based inhibitor PBD150 exhibit an inhibitory constant in the low nanomolar range [56]. Due to the linkage of an active site optimized backbone group to the metal binding group imidazole, the $K_{\mathrm{i}}$ value was drastically decreased by a factor of $\sim 2000$. Accordingly, linkage of the metal binding group imidazole with the backbone group N-(3,4-dimethoxyphenyl)thiourea increased binding affinity. The carbohydrazide function turned out to be a potential better candidate for complexation of the catalytic zinc ion in the active site of hQC compared to imidazole due to stronger binding. Thus, combining the carbohydrazide function with N-(3,4-dimethoxyphenyl)thiourea via a linker should result in theory in a potentially stronger inhibitor for hQC compared to PBD150. In addition, due to this modification, potency and selectivity of the new designed inhibitor should be further optimized. 


\section{Summary and Final Discussion}

Many neuropeptides are post-translationally modified at the N-terminus to increase their bioactivity. This post-translational modification is in many cases essential, for instances for the formation of a physiological active structure, binding to specific receptors or to increase the half-life time of a neuropeptide $[24,29]$. One enzyme family, which is involved in the post-translational modification of N-terminal amino acids are glutaminyl cyclases. Glutaminyl cyclase occurs in the Animalia, Plantae and Bacteria kingdom, in which it physiologically catalyzes the formation of pyroglutamic acid from N-terminal glutaminyl precursors. Interestingly, this apparent homology between mammalian, plant and bacterial glutaminyl cyclases is not reflected in the primary structure (Fig. 7.1). Plant and bacterial glutaminyl cyclases have a highly similar overall structure and thereby obviously representing an individual family of enzymes [128], while the highly homologous mammalian glutaminyl cyclases share structural similarities to bacterial aminopeptidases [11]. The structures of glutaminyl cyclase from Zymomonas mobilis (bacteria) [129] and Carica papaya (plant) [130] exhibit a characteristic $\beta$-propeller fold composed of five $\beta$-sheets with antiparallel $\beta$-strands, which are arranged around a central axis and a short $\alpha$-helical turn. The $\beta$-propeller fold showed a high resistance to chemical, acid and thermal denaturation [131]. Furthermore, an octahedral coordinated metal ion is located in the core of both proteins. It is reported in literature that the central metal ion could be either zinc [130] or calcium [132]. In contrast, glutaminyl cyclases of mammals (Homo sapiens and Mus musculus) and invertebrates (Drosophila melanogaster) possess a globular $\alpha / \beta$ hydrolase fold $[20,22,133]$ harboring a tetrahedral coordinated catalytic zinc ion within their active sites. Zinc is a very attractive catalyst for biological systems due to e.g. its flexible coordination geometry, the fast ligand exchange, the lack of redox activity, and its role as Lewis acid. In addition, physiological functions of plant and bacterial glutaminyl cyclases differ from that of animal glutaminyl cyclases. For example, glutaminyl cyclase from Carica papaya is probably involved in the production of ammonium ions in the plant pathogen defense [134], whereas animal glutaminyl cyclases play a role in the synthesis of pyroglutamyl modified neuropeptides or hormones [54]. Accordingly, evolution of plant, bacterial and animal glutaminyl cyclases apparently led to functionally similar enzymes, but they differ in their tertiary structure and physiological function. 
Table 7.1: Overview of kinetic parameters of glutaminyl cyclase from different domains of life catalyzing $\mathrm{N}$-terminal glutaminyl substrates. All measurements were performed at $30^{\circ} \mathrm{C}$ in 50 $\mathrm{mM}$ Tris/ $\mathrm{HCl}, \mathrm{pH} 8.0$

\begin{tabular}{|c|c|c|c|}
\hline \multicolumn{4}{|c|}{ Human glutaminyl cyclase } \\
\hline Peptide & $\begin{array}{l}k_{\text {cat }} \\
{\left[\mathrm{s}^{-1}\right]}\end{array}$ & $\begin{array}{c}K_{\mathrm{M}} \\
{[\mathrm{mM}]}\end{array}$ & $\begin{array}{c}k_{\text {cat }} / K_{\mathrm{M}} \\
{\left[\mathrm{s}^{-1} \mathrm{mM}^{-1}\right]}\end{array}$ \\
\hline QQ & $24.8 \pm 0.13$ & $0,14 \pm 0.01$ & $233.5 \pm 14.6$ \\
\hline QFRH & $68.6 \pm 0.32$ & $0.27 \pm 0.01$ & $254.1 \pm 29.6$ \\
\hline $\mathrm{Q}-\beta \mathrm{NA}$ & $20.4 \pm 0.87$ & $0.12 \pm 0.01$ & $170.0 \pm 20.6$ \\
\hline \multicolumn{4}{|c|}{ Mammal QC (Mus musculus) ${ }^{\mathrm{A}}$} \\
\hline Peptide & $\begin{array}{l}k_{\text {cat }} \\
{\left[\mathrm{s}^{-1}\right]}\end{array}$ & $\begin{array}{c}K_{\mathrm{M}} \\
{[\mathrm{mM}]}\end{array}$ & $\begin{array}{c}k_{\text {cat }} / K_{\mathrm{M}} \\
{\left[\mathrm{s}^{-1} \mathrm{mM}^{-1}\right]}\end{array}$ \\
\hline QQ & $32.0 \pm 1.00$ & $0,15 \pm 0.01$ & $213.0 \pm 8.0$ \\
\hline QFRH & n.d. & n.d. & n.d. \\
\hline $\mathrm{Q}-3 \mathrm{NA}{ }^{\mathrm{B}}$ & $22.0 \pm 1.00$ & $0.040 \pm 0.004$ & $550.0 \pm 30.0$ \\
\hline \multicolumn{4}{|c|}{ Invertebrate QC (Drosophila melanogaster $)^{\mathrm{A}}$} \\
\hline Peptide & $\begin{array}{c}k_{\text {cat }} \\
{\left[\mathrm{s}^{-1}\right]} \\
\end{array}$ & $\begin{array}{c}K_{\mathrm{M}} \\
{[\mathrm{mM}]} \\
\end{array}$ & $\begin{array}{c}k_{\text {cat }} / K_{\mathrm{M}} \\
{\left[\mathrm{s}^{-1} \mathrm{mM}^{-1}\right]} \\
\end{array}$ \\
\hline QQ & $7.4 \pm 0.05$ & $0,94 \pm 0.05$ & $7.9 \pm 0.2$ \\
\hline QFRH & $13.9 \pm 0.4$ & $0.5 \pm 0.02$ & $150.0 \pm 0.9$ \\
\hline $\mathrm{Q}-3 \mathrm{NA} \mathrm{B}^{\mathrm{B}}$ & $1.1 \pm 0.1$ & $0.15 \pm 0.01$ & $7.4 \pm 0.3$ \\
\hline \multicolumn{4}{|c|}{ Plant QC $(\text { Carica papaya })^{\mathrm{A}}$} \\
\hline Peptide & $\begin{array}{c}k_{\text {cat }} \\
{\left[\mathrm{s}^{-1}\right]}\end{array}$ & $\begin{array}{c}K_{\mathrm{M}} \\
{[\mathrm{mM}]}\end{array}$ & $\begin{array}{c}k_{\mathrm{cat}} / K_{\mathrm{M}} \\
{\left[\mathrm{s}^{-1} \mathrm{mM}^{-1}\right]}\end{array}$ \\
\hline QQ & $43.2 \pm 0.70$ & $0,044 \pm 0.003$ & $982.0 \pm 51.0$ \\
\hline QFRH & n.d. & n.d. & n.d. \\
\hline $\mathrm{Q}-3 \mathrm{NA}^{\mathrm{B}}$ & $51.4 \pm 1.4$ & $0.038 \pm 0.003$ & $1353.0 \pm 70.0$ \\
\hline \multicolumn{4}{|c|}{ Bacterial QC (Zymomonas mobilis) ${ }^{\mathrm{A}}$} \\
\hline Peptide & $\begin{array}{c}k_{\text {cat }} \\
{\left[\mathrm{s}^{-1}\right]} \\
\end{array}$ & $\begin{array}{c}K_{\mathrm{M}} \\
{[\mathrm{mM}]}\end{array}$ & $\begin{array}{c}k_{\mathrm{cat}} / K_{\mathrm{M}} \\
{\left[\mathrm{s}^{-1} \mathrm{mM}^{-1}\right]}\end{array}$ \\
\hline QQ & $0,27 \pm 0.05$ & $20.4 \pm 5.60$ & $0.0013 \pm 0.0004$ \\
\hline QFRH & n.d. & n.d. & n.d. \\
\hline $\mathrm{Q}-3 \mathrm{NA}^{\mathrm{B}}$ & $2.7 \pm 0.40$ & $0.04 \pm 0.003$ & $6.6 \pm 1.1$ \\
\hline
\end{tabular}

\footnotetext{
A) Kinetic parameters from [54, 101, 129, 135], respectively.

B) Substrate inhibition detected

n.d. $=$ not determined
}

In order to investigate the impact of the structural differences of glutaminyl cyclase for each class, literature and experimentally determined kinetic parameters for selected $\mathrm{N}$-terminal glutaminyl substrates (Gln-Gln and Q-BNA) were compared with each other (Tab. 7.1). 




Figure 7.1: Overview of the different protein folds of glutaminyl cyclase from animalia, plantae and bacteria kingdom. (A) Structural alignment of glutaminyl cyclase from Carica papaya (plant) and Zymomonas mobilis (bacteria). B Structural alignment of glutaminyl cyclase from Homo sapiens (mammal), Mus musculus (mammal) and Drosophila melanogaster (invertebrate). Structures for alignments were taken from PDB entries 2FAW, 3NOM, 3SI1 and 4FAI.

Kinetic characterization of the N-terminal glutaminyl substrates catalyzed by animal, plant and bacterial glutaminyl cyclases showed differences in their catalytic efficiencies. As expected, mammalian glutaminyl cyclases (Homo sapiens and Mus musculus) exhibit comparable catalytic efficiencies with nearly identical turnover number and Michaelis-Menten constants. In case of the glutaminyl cyclas from invertebrates (Drosophila melanogaster) a 30-fold decreased catalytic efficiency compared to mammalian glutaminyl cyclases was observed. This was quite surprising, because glutaminyl cyclases of invertebrates share the same structural fold compared to mammalian glutaminyl cyclases. The impact of different catalytic efficiencies for N-terminal glutaminyl substrates is probably caused by differences in the active site environment, thereby influencing catalysis directly. Interestingly, catalytic efficiency of plant glutaminyl cyclase (Carica papaya) is increased by a factor of four compared to hQC. Glutaminyl cyclase from Carica papaya compared to hQC catalyzes the cyclization of the dipeptide Gln-Gln or the fluorogenic substrate (Q-BNA) harboring an N-terminal glutamine with higher efficiency. It was reported that with increasing chain length of the substrate the selectivity of hQC increases, which is in contrast to glutaminyl cyclase from Carica papaya [54]. Additionally, hQC was more specific concerning the structure of the peptide chain compared to glutaminyl cyclase from Carica papaya expressed by lower Michaelis-Menten constants towards peptides with proline or partially cyclic peptides at the second N-terminal amino acid position. A pos- 
sible explanation could be the structural differences of secondary substrate binding sites in both enzymes, suggesting different interactions of the N-terminal glutaminyl substrate with the active site of the enzyme. In case of the bacterial glutaminyl cyclase (Zymomonas mobilis) a drastically decreased catalytic efficiency for N-terminal glutaminyl substrates compared to hQC was observed (Tab. 7.1). Glutaminyl cyclase from Zymomonas mobilis shows maximum activity in the mild acidic range with $\mathrm{p} K_{a 1}=5.25 \pm 0.07$ and $\mathrm{p} K_{a 2}$ $=6.87 \pm 0.07$ [129], which is in contrast to other glutaminyl cyclase enzymes displaying maximum activity in the mild alkaline range. The shift into a mild acidic $\mathrm{pH}$ range for maximal glutaminyl cyclase activity is in accordance with the acidic $\mathrm{pH}$ growth optimum of Zymomonas mobilis. However, the physiological function of glutaminyl cyclase in $Z y$ momonas mobilis has not been elucidated so far. In addition, it is important to mention that the catalytic mechanism of plant and bacterial glutaminyl cyclases is lacks investigation to date. It is still under debate if the octahedral coordinated metal ion is necessary for catalysis or only for stabilization of the tertiary structure. Further the question must be addressed how the binding of the substrate can occur.

Besides pyroglutaminyl modified hormones from animals at N-terminal glutaminyl precursors, it was also reported that amyloidogenic peptides exhibit a cyclized N-terminus. Patients suffering from Alzheimer's disease have an increased deposition of amyloid plaques within their brains, which mainly consist of modified amyloidogenic peptides. Interestingly, recent studies utilizing a discontinuous kinetic assay have shown that hQC catalyzes the formation of pyroglutamic acid at the N-terminus of amyloidogenic peptides [5] and thus might play a significant role in the initial phase of amyloid plaque formation. Moreover, amyloidogenic peptides harbor an N-terminal glutamic acid instead of an Nterminal glutamine implicating that hQC is also capable of cyclizing N-terminal glutamyl substrates. Involvement of hQC in Alzheimer's disease and catalysis of N-terminal glutamyl substrates was demonstrated by a genetically upregulated expression of glutaminyl cyclase in the cortices of transgenic mice, resulting in an accelerated formation of amyloid plaques [115]. Furthermore, plaque formation and accumulation of amyloidogenic peptides was drastically suppressed by inhibition of glutaminyl cyclase by the PBD150 inhibitor. One goal in this presented study was a comparative study of the catalytic properties of hQC while catalyzing N-terminal glutaminyl (physiological reaction) and 
glutamyl (pathophysiological reaction) substrates and to decipher the reaction mechanism for both cases. During this study hQC was characterized kinetically, spectroscopically and structurally in presence of N-terminal glutaminyl/glutamyl substrates. To characterize kinetically the physiological and pathophysiological reaction catalyzed by hQC a new continuous activity assay using isothermal titration calorimetry was developed. This is the first time characterizing an N-terminal glutamyl substrate with a continuous activity assay. As expected, formation of pyroglutamic acid from an N-terminal glutamic acid was drastically decelerated compared to an N-terminal glutamine. Conversion of N-terminal glutaminyl/glutamyl substrates catalyzed by hQC differed by a factor of $\sim 5200$ in their turnover number (Fig. 6.7). Additionally, hQC displayed a $\mathrm{pH}$ dependency for conversion of N-terminal glutaminyl/glutamyl substrates. In case of the physiological reaction an alkaline $\mathrm{pH}$ value was favored, while the pathophysiological reaction only occurred at acidic $\mathrm{pH}$ conditions. In order to elucidate the difference in the kinetic profiles for the physiological and pathophysiological reaction of glutaminyl cyclase further investigations by X-ray crystallography and different spectroscopic methods were performed. Especially the active site with its catalytic zinc ion of glutaminyl cyclase in presence of N-terminal glutaminyl/glutamyl substrates was in focus of analysis. Crystal structures of hQC and isoDromeQC (data from Alexander Piontek) in complex with N-terminal glutaminyl/glutamyl substrates clearly showed different binding modes of both substrates within the active site. These observations were supported by stopped flow UV/Vis spectroscopy (Co(II)-hQC) and rapid freeze quench EPR (Co(II)-isoDromeQC) (data from Alexander Piontek) experiments. Analysis of the different experimental data sets revealed that coordination of N-terminal glutaminyl substrates to the catalytic zinc ion was monodentally, resulting in a tetrahedral coordination geometry of the central metal ion. On the contrary, coordination of N-terminal glutamyl substrates to the catalytic zinc ion was bidentally, leading to a five-fold coordinated central metal ion. In addition, crystal structure of the active site of isoDromeQC showed an alternative conformation for a coordinated glutamic acid, indicating a carboxylate shift. The carboxylate shift caused a change in the coordination geometry from a five-fold (bidental coordinated glutamic acid) to a tetrahedral coordination (monodental coordinated glutamic acid). This difference in the binding mode of potential substrates and the coordination geometry of the catalytic zinc ion might explain difference in the kinetic profiles of N-terminal glutaminyl/glutamyl 
substrates. A prerequisite for cyclization of N-terminal glutamines and glutamic acids is a tetrahedral coordination of the zinc and a monodental coordinated substrate due to release of the leaving group. Accordingly, no reaction would occur for a five-fold coordinated central metal ion with a bidental coordinated substrate. Therefore, a change in the coordination mode (bi- to monodentate) is necessary for catalysis, which is induced by a pH-dependent carboxylate shift.

A typical carboxylate shift is characterized by a change in the coordination form of a carboxylate group and it was reported that such a shift could be kinetically important in carboxylate containing metalloproteins [109]. Carboxylate group coordinating amino acids are glutamic and aspartic acids, which display identical $\mathrm{p} K_{a}$ values. The change in the binding mode typically happens from a bidentate to monodentate coordination (or vice versa) in mononuclear systems after ligand binding. To provide an efficient mechanism to lower the activation barriers for ligand binding and catalysis, a nearly constant coordination number should be maintained throughout an entire catalytic pathway of a given enzyme, which is accomplished by the rearrangement of the carboxylate group [136]. The result is basically an interchange of ligands and extremely high flexibility of the zinc coordination sphere, which is the case for the active site of hQC in presence of a substrate. Density functional theory (DFT) calculations showed that the exact combination of amino acid residues at the metal coordination sphere, including the substrate after binding, determine this effect [136], which is even further influenced in biological systems by the specific enzymatic environment surrounding each metallic center [137]. Moreover, the following conditions are necessary for a preferred bidentate carboxylate binding over the monodentate mode [137]: (1) the coordination number of the metal cation needs to be high; (2) the metal cation must have good Lewis acid properties and should thereby easily accept the charge from the second carboxylate oxygen; (3) negatively charged ligands in the metal complex should not neutralize the positive charge of the metal cation; (4) the metal cation should easily accommodating bulky protein main/side chain dipoles; (5) The metal cation should be relatively large; and (6) poor or nonexistent hydrogen bond donors in the first or second coordination shell, which could potentially fixate other binding modes. All mentioned points are fulfilled in case of the catalytic zinc ion with a coordinated N-terminal glutamyl substrate within the active site of hQC at alkaline $\mathrm{pH}$ 
conditions. However, if switching to acidic $\mathrm{pH}$ conditions one oxygen atom of the carboxylate group gets protonated, resulting in a hydroxy group and loss of the negative charge. Protonation of the oxygen atom at the $\gamma$-carboxylic moiety of the N-terminal glutamic acid of the substrate can be accomplished by accepting a proton from the solvent or from a nearby proton donating amino acid residue (Fig. 6.21). Due to this protonation the N-terminal glutamate can only coordinate monodentally to the zinc and thereby changing the coordination number of the metal complex. In summary, a pH-dependent carboxylate shift might explain conversion of glutamic acids to pyroglutamic acids at the $\mathrm{N}$-terminus of amyloidogenic peptides catalyzed by hQC. Carboxylate shifts regulating enzymatic activity or substrate binding were already reported for different enzymes, for example in case of methane monooxygenase [109-111], farnesyltransferase [138] and the R2 subunit of ribonucleotide reductase [139].

Involvement of hQC activity in the modification of amyloidogenic peptides by catalyzing N-terminal glutamate to pyroglutamic acid via an intramolecular cyclization reaction opens up further possibilities for the use of effectors for hQC with a pharmacological objective. The primary interest is the development of a potent hQC inhibitor with the aim to suppress the formation of toxic pyroglutamyl modified amyloidogenic peptides by targeting directly the catalytic metal ion within the active site. Inhibiting the hQC activity in neuronal cells of Alzheimer's disease patients might block the N-terminal pyroglutamyl formation at amyloidogenic peptides. As a result, the unmodified amyloidogenic peptide species would not be stabilized against proteolysis anymore. Accordingly, an increased degradation of the unmodified amyloidogenic peptides would be the result, which in turn would contribute to a decelerated toxic plaque formation within the brain of Alzheimer's disease patients. However, permanent inhibition of hQC activity might not be essential due to the slow turnover rate of hQC catalyzing formation of pyroglutamic acid at N-terminal glutamyl substrates in its pathophysiological reaction compared to its physiological reaction. Thus, a partial inhibition of hQC activity by a potent reversible inhibitor might have a therapeutic effect on toxic plaque formation, while the physiologic reaction is not completely inhibited and could occur to a certain degree. Assuming that the formation of the first toxic plaque deposits need decades, a partial inhibition of the hQC would already delay the onset of the disease significantly, whereas the physiological reac- 
tion at the N-terminus of small peptides and hormones could theoretically occur, thereby increasing their bioactivity. However, there is still no reliable diagnostic method to predict the onset of Alzheimer's disease at an individual. Apparatus examination methods as magnetic resonance imaging for instance provide no clear evidence of precursors or early forms of Alzheimer's disease. An exception of an early diagnosis of Alzheimer's disease at an individual is a genetic test, in which changes in the genetic material can be attributed to the neurodegenerative disease. Unfortunately, they account for only less than $5 \%$ of all cases.

First investigations revealed that imidazole-N1-derivatives are a potential metal binding group for the catalytic zinc of hQC [23]. Based on these finding the imidazole-based metal binding group was linked with a specifically designed and active site adjusted hydrophobic backbone group, resulting in a potent inhibitor for hQC in the low nanomolar range [56]. Preclinical studies with the imidazole-based inhibitor in transgenic mice showed a positive inhibition of glutaminyl cyclase [115]. However, approximately one-third of proteins within the human system are metalloproteins [140], which are involved in diverse functions in vivo, for example in regulation of the blood $\mathrm{pH}$. Concerning the importance of the diverse functions carried out by metalloproteins, a misregulation by indiscriminate binding of inhibitors to metal-dependent enzymes could cause significant changes in the homeostatic balance within the human metabolism. A search for metalloprotein inhibitors in the Protein Data Bank (PDB) showed that common metal binding groups were hydroxamic acid, carboxylic acids, thiols, phosphonates and imidazole derivatives. Screening for off-target inhibition by testing a variety of known metalloenzyme inhibitors, which harbor common metal binding groups, against a representative panel of metalloproteins showed a limited indiscriminate binding of the inhibitors [118]. Furthermore, off-target inhibition was greater among metalloenzymes incorporating the same catalytic metal, active site structure and functionality. Another aspect during this study was to design a unique and non-imidazole-based metal binding group with high affinity and selectivity for the catalytic zinc ion of hQC to prevent or, at least, to diminish off-target inhibition. Screening of different compounds revealed L-glutamic acid $\gamma$-hydrazide $(\mathrm{E}(\gamma$-hydrazide $))$ as a potential metal binding group candidate for coordination to the catalytic zinc ion of hQC with an inhibitory constant in the low micromolar range. Kinetic characterization of L-glutamic 
acid $\gamma$-hydrazide showed a higher affinity to the catalytic zinc ion compared to imidazole, thereby could serve as a potential better metal binding group. Crystal structure of hQC in complex with the inhibitor confirmed tight binding of the metal binding group by the establishment of several interactions of the carbohydrazide function with the catalytic zinc ion and surrounding amino acid residues of the active site of hQC. Interestingly, the carbohydrazide function bound bidentally to the catalytic zinc ion, resulting in a five-fold coordinated central metal ion. In addition, distorted bond angles within the metal complex were observed, indicating a trigonal bipyramidal and a square pyramidal molecular geometry. This observation of the distorted bond angles could be caused by a Berry pseudorotation or due to relative fixation of the metal binding group by formation of hydrogen bonds between the carbohydrazide function and surrounding amino acid residues within the active site. However, obtained experimental data clearly showed that $\mathrm{E}(\gamma$-hydrazide) could be used as a new potential metal binding group in case of hQC. Furthermore, no explicit cases for metalloenzymes inhibited by a carbohydrazide function were so far described in literature. It might be possible that selectivity is additionally increased, which would consequently diminish off-target inhibition.

In addition to the development of inhibitors for hQC it was considered that the $\beta$-secretase is also a potential drug target candidate in the treatment of Alzheimer's disease, but the current state of drug development is still in an early phase. The $\beta$-secretase is together with the $\alpha$-secretase involved in the proteolytic processing of the amyloid precursor protein $(\mathrm{APP})$, resulting in different amyloidogenic peptides varying in length. However, in 2012 the German company Merck was successful in developing an inhibitor, namely MK-8931, for the $\beta$-secretase. MK-8931 reduced toxic plaque deposits within the cerebral spinal fluid (CSF) in healthy human subjects and was further investigated in clinical phases II and III to elucidate its efficacy and to expulse possible side effects. Another idea is the combination of hQC inhibitors with effectors of secretase activities at low compound concentrations, which could reduce potential side effects of each species to obtain a maximal therapeutic effect. While the recent inhibitor studies try to prevent toxic plaque formation other studies deal with the removal of already manifested accumulated amyloidogenic peptides by immunotherapy. It was reported that passive immunization with monoclonal and polyclonal anti-amyloid-beta antibodies leads to improvement of Alzheimer's disease 
pathology in mouse models $[141,142]$. In summary, combination of both therapeutic projections might be important steps in the future treatment of Alzheimer's disease.

In summary, during this study the role of glutaminyl cyclase and its role in neurogegenrative diseases was investigated. Therefore, glutaminyl cyclase from Homo sapiens was heterologously expressed and purified to homogeneity. A continuous calorimetric activity assay was developed to kinetically characterize the cyclization reaction directly of $\mathrm{N}$ terminal glutaminyl/glutamyl substrates catalyzed by hQC. In addition, a reaction model of the cyclization of N-terminal glutamyl substrates based on mechanistic and structural data (e.g. stopped flow UV/Vis spectroscopy, EPR spectroscopy and X-ray crystallography) was proposed. The reaction model includes a carboxylate shift to explain the differences in catalytic efficiencies compared to N-terminal glutaminyl substrates. Furthermore, a first step in the development of a hQC inhibitor was successful by utilizing a carbohydrazide function as a potential metal binding group. 


\section{Appendix}

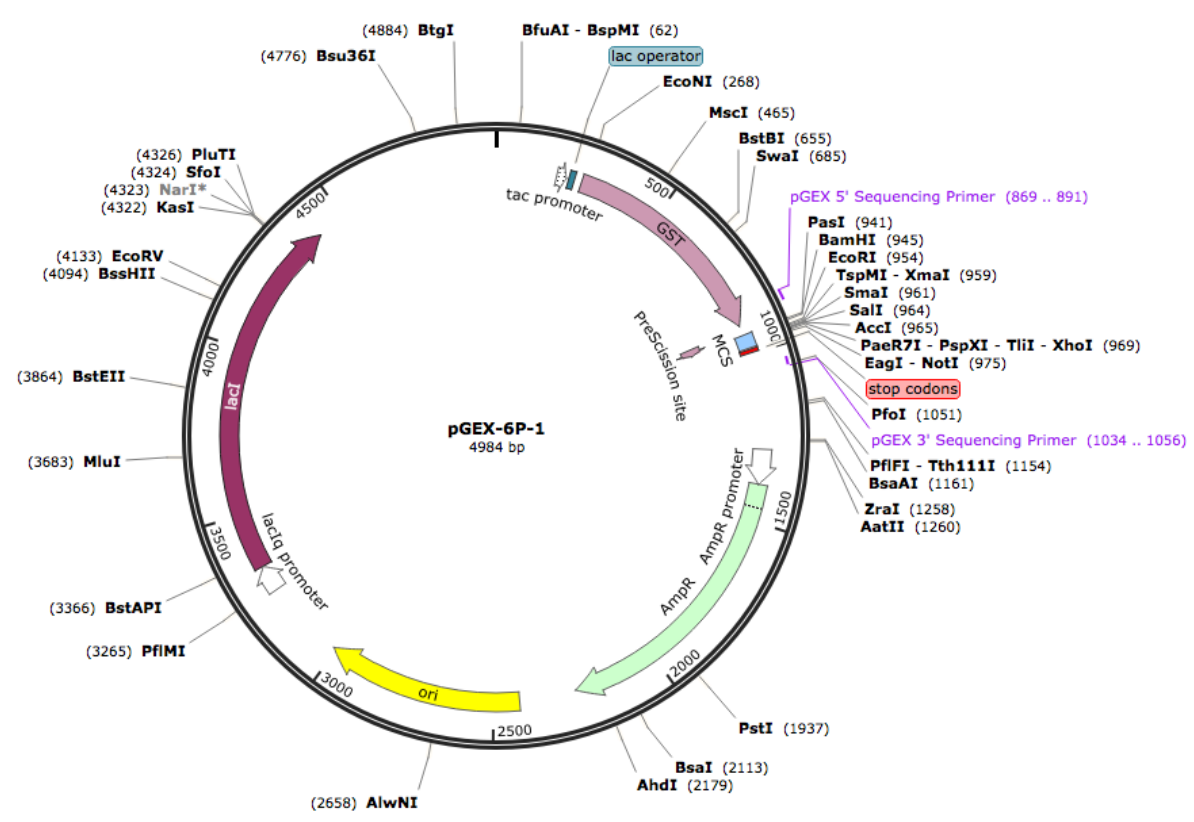

Figure 8.1: Vector map of pGEX-6P-1. Map was downloaded from the SnapGene server.

ILGYWKIKGLVQPTRLLLEYLEEKYEEHLYERDEGDKWRNKKFELGLEFPNLPYYIDGDVKLTQSMAIIRYIADKHNMLGGCPKERAEISMLEGA

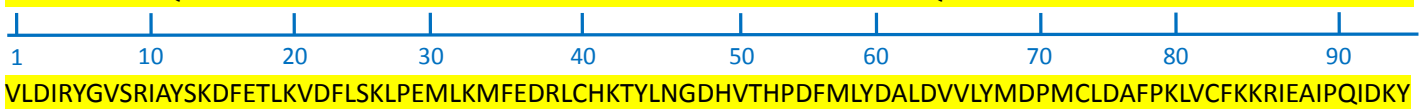

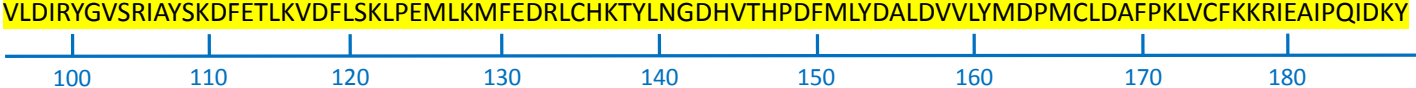

LKSSKYIAWPLQGWQATFGGGDHPPKSDLEVLFQGPAWPEEKNYHQPAILNSSALRQIAEGTSISEMWQNDLQPLLIERYPGSPGSYAARQH

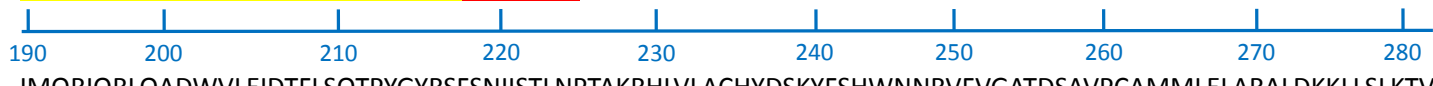

IMQRIQRLQADWVLEIDTFLSQTPYGYRSFSNIISTLNPTAKRHLVLACHYDSKYFSHWNNRVFVGATDSAVPCAMMLELARALDKKLLSLKTV

\begin{tabular}{ccccccccc}
$\mid$ & $\mid$ & $\mid$ & $\mid$ & $\mid$ & $\mid$ & $\mid$ & $\mid$ & $\mid$ \\
\hline 290 & 300 & 310 & 320 & 330 & 340 & 350 & 360 & 370
\end{tabular}

SDSKPDLSLQLIFFDGEEAFLHWSPQDSLYGSRHLAAKMASTPHPPGARGTSQLHGMDLLVLLDLIGAPNPTFPNFFPNSARWFERLQAIEHE

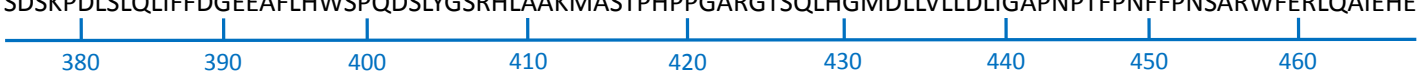

LHELGLLKDHSLEGRYFQNYSYGGVIQDDHIPFLRRGVPVLHLIPSPFPEVWHTMDDNEENLDESTIDNLNKILQVFVLEYLHL

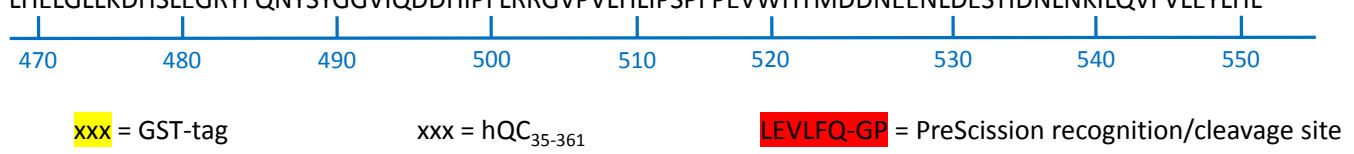

Figure 8.2: Amino acid sequence of the fusion protein GST-hQC $35-361$. 


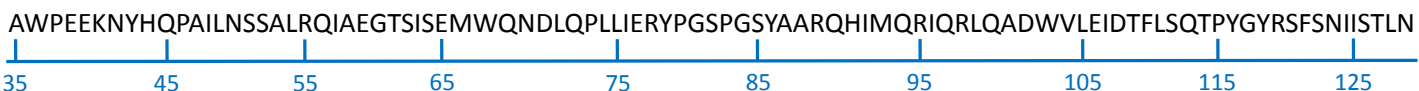

PTAKRHLVLACHYDSKYFSHWNNRVFVGATDSAVPCAMMLELARALDKKLLSLKTVSDSKPDLSLQLIFFDGEEAFLHWSPQDSLYGSRHLAA

\begin{tabular}{cccccccc|c}
\hline & & & & & & & & \\
\hline 135 & 145 & 155 & 165 & 175 & 185 & 195 & 205
\end{tabular}

KMASTPHPPGARGTSQLHGMDLLVLLDLIGAPNPTFPNFFPNSARWFERLQAIEHELHELGLLKDHSLEGRYFQNYSYGGVIQDDHIPFLRRG

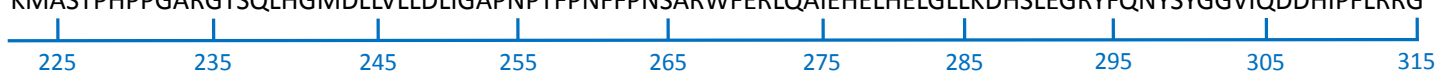

VPVLHLIPSPFPEVWHTMDDNEENLDESTIDNLNKILQVFVLEYLHL

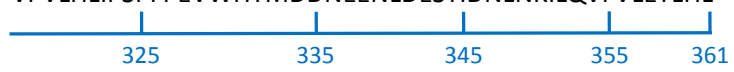

Figure 8.3: Amino acid sequence of $\mathrm{hQC}_{35-361}$.

A

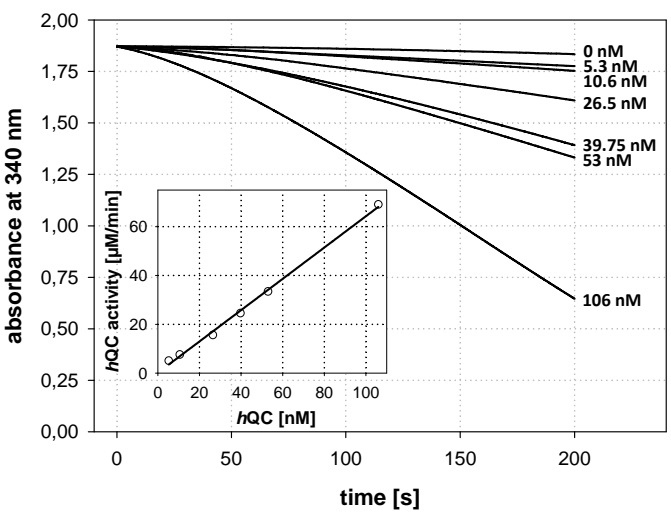

B



Figure 8.4: Kinetic characterization of hQC utilizing the spectroscopic coupled assay. (A) Progress curves of the cyclization of the dipeptide Gln-Gln (QQ) with varying concentration of hQC. The inset shows the linear relationship of hQC concentration and the observed activity. (B) Substrate dependency of hQC activity with determined kinetic constants. 
A


B



D

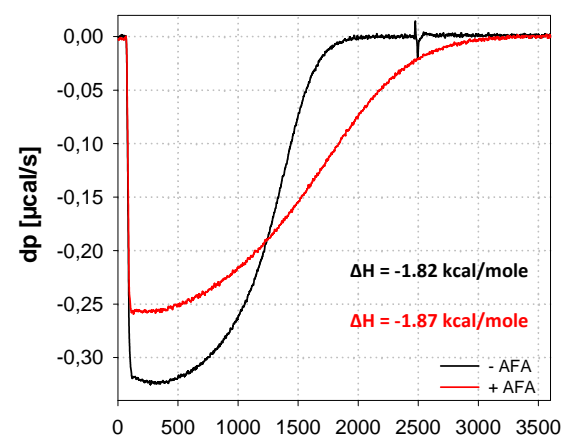

Figure 8.5: Controls for determination of the overall molar reaction enthalpy of hQC after substrate depletion under changing conditions. Measurements were performed in $50 \mathrm{mM}$ Tris/ $\mathrm{HCl}$, $\mathrm{pH}$ 8.0. (A) Overview of substrate consumption and product formation over time in a standard reaction utilizing the continuous calorimetric activity assay. (B) Detected heat release of the cyclization reaction at constant ratio of enzyme and substrate concentration. (C) Detected heat release with varying substrate, but constant enzyme concentration. (D) Heat formation in presence and absence of an additional ligand. $\Delta \mathrm{H}$ is shown for each reaction respectively.

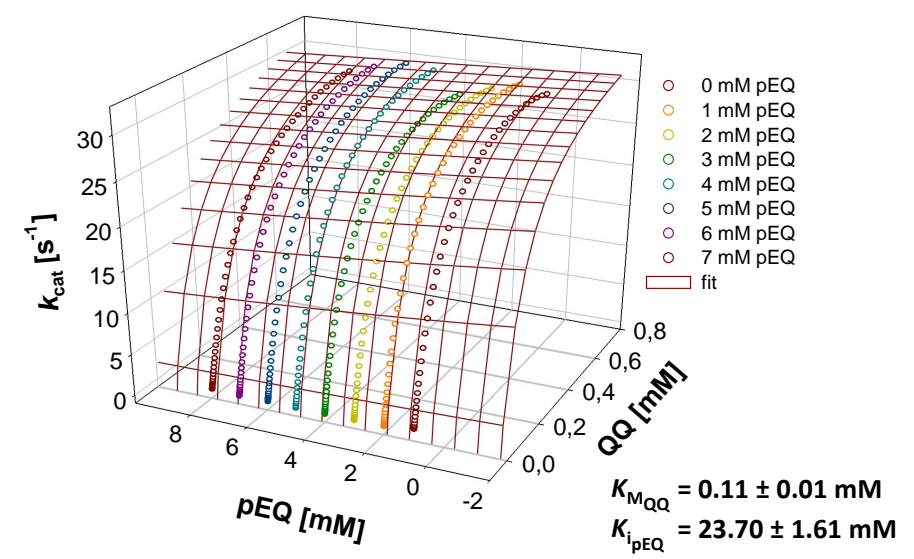

Figure 8.6: Three-dimensional analysis of substrate depletion and product inhibition for hQC utilizing the continuous calorimetric activity assay. Obtained data points were fitted according to Eq. 15. 
A



C

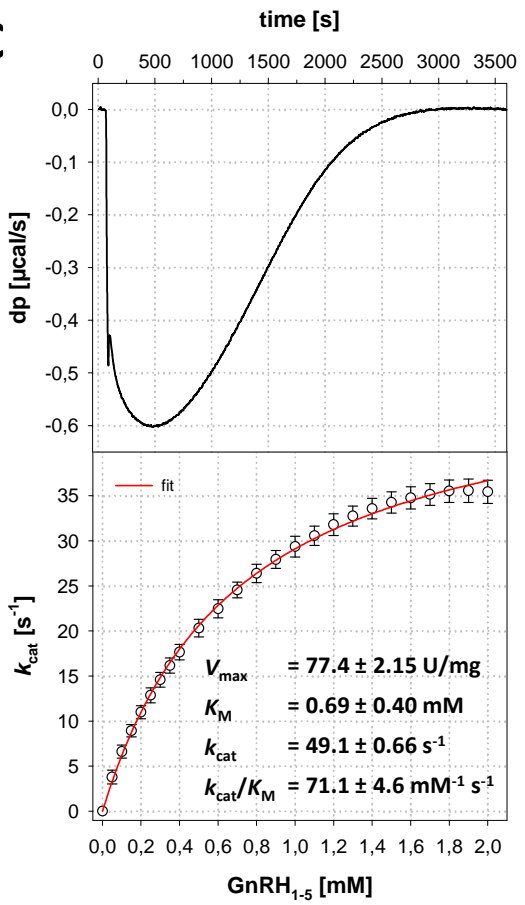

B



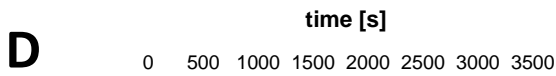

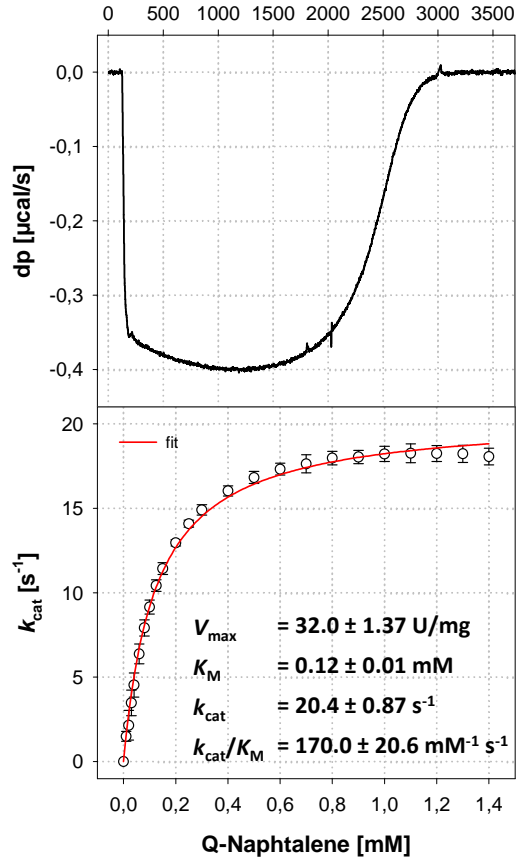

Figure 8.7: hQC catalyzed cyclization reaction of N-terminal glutaminyl substrates measured via the continuous calorimetric activity assay. Kinetic characterization of (A) neurotensin ${ }_{1-5}$, (B) orexin $\mathrm{A}_{1-5}$, (C) $\mathrm{GnRH}_{1-5}$ and (D) Q-BNA. The determined kinetic parameters are depicted, respectively. All measurements were performed in $50 \mathrm{mM}$ Tris $/ \mathrm{HCl}, \mathrm{pH} 8.0$. 
A



B

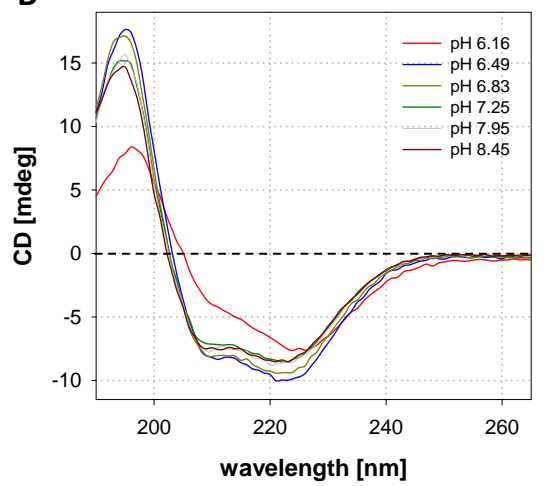

Figure 8.8: Absorption of $\mathrm{Co}(\mathrm{II})$-hQC in dependency of the cobalt concentration. (A) Kinetic constants were obtained employing the continuous calorimetric assay. Measurements were performed in $0.05 \mathrm{M}$ acetic acid, $0.05 \mathrm{M} \mathrm{MES}$ and $0.1 \mathrm{M}$ Tris at $30^{\circ} \mathrm{C}$ utilizing the reference substrate QQ.

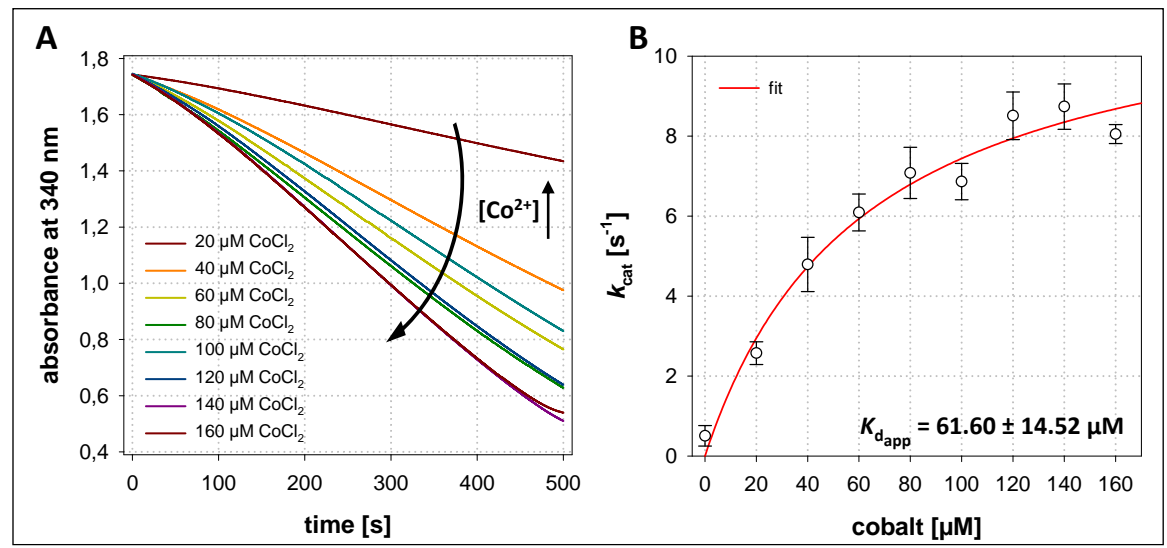

Figure 8.9: Kinetic studies of cobalt association and dissociation. Reconstitution was performed in $50 \mathrm{mM} \mathrm{NaH} \mathrm{PO}_{4}, 100 \mathrm{mM} \mathrm{NaCl}, \mathrm{pH} 6.8$, and activity measurements in $50 \mathrm{mM}$ Tris/HCl, $\mathrm{pH}$ 8.0. A standard activity reaction consisted of $40 \mu \mathrm{M}$ apoenzyme supplemented with increasing cobalt concentrations and incubated for $15 \mathrm{~min}$ at room temperature. Subsequently, the catalytic activity was measured using the spectroscopic coupled assay. (A) Progress curves of Co(II)-hQC in dependency of the cobalt concentration measured with the spectroscopic coupled assay. (B) Determination of the dissociation constant by plotting obtained turnover number from the activity assay against cobalt concentration. 




Figure 8.10: Active site alignment of hQC and isoDromeQC. Zinc ions are depicted as black spheres, whereas hQC (grey) and isoDromeQC (green) are shown in cartoon style. Corresponding residue numbers are depicted, respectively. Data for isoDromeQC was taken from Alexander Piontek

A

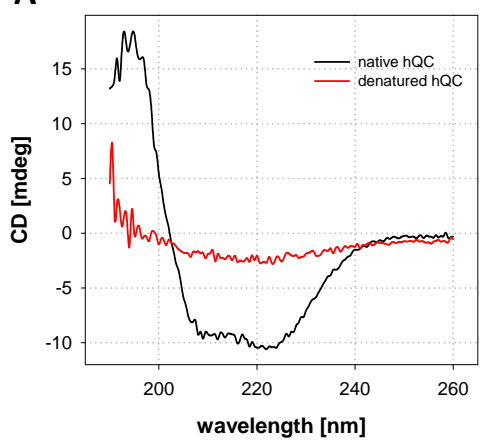

B

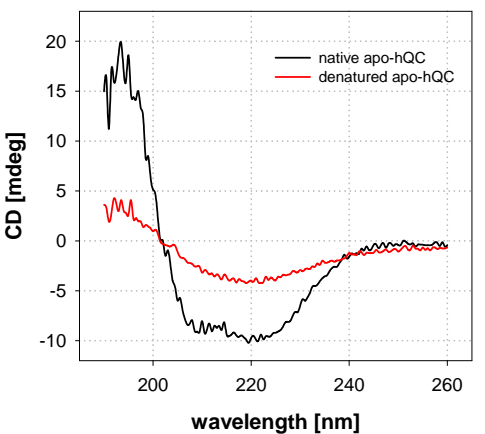

C



Figure 8.11: CD-spectra of hQC, Co(II)-hQC and apoenzyme before and after thermal denaturation. CD-spectra for (A) hQC, (B) $\mathrm{Co}(\mathrm{II})-\mathrm{hQC}$ and (C) apoenzyme are shown respectively. 


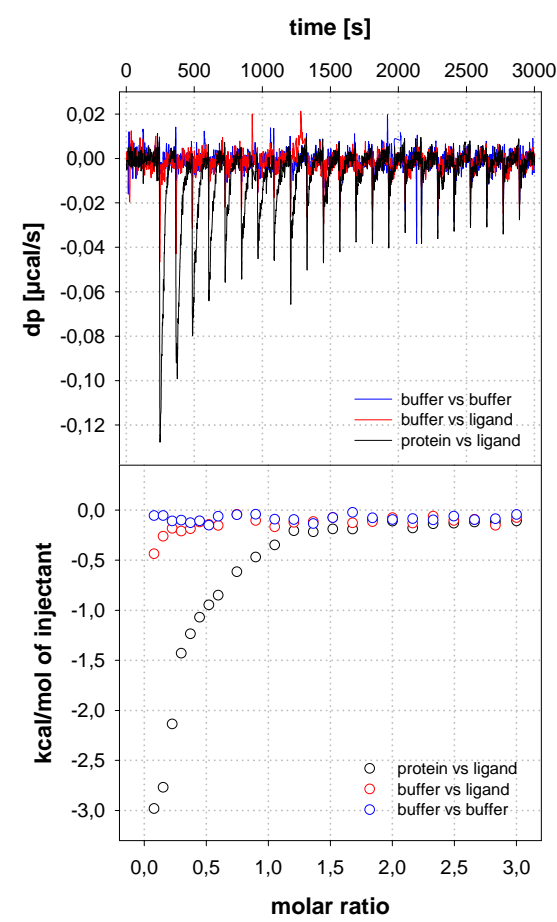

Figure 8.12: Thermodynamic characterization of binding of $\mathbf{E}(\gamma$-hydrazide)FA to hQC isothermal titration calorimetry. Measurement was performed in $50 \mathrm{mM}$ Tris/HCl, $\mathrm{pH}$ 8.0.

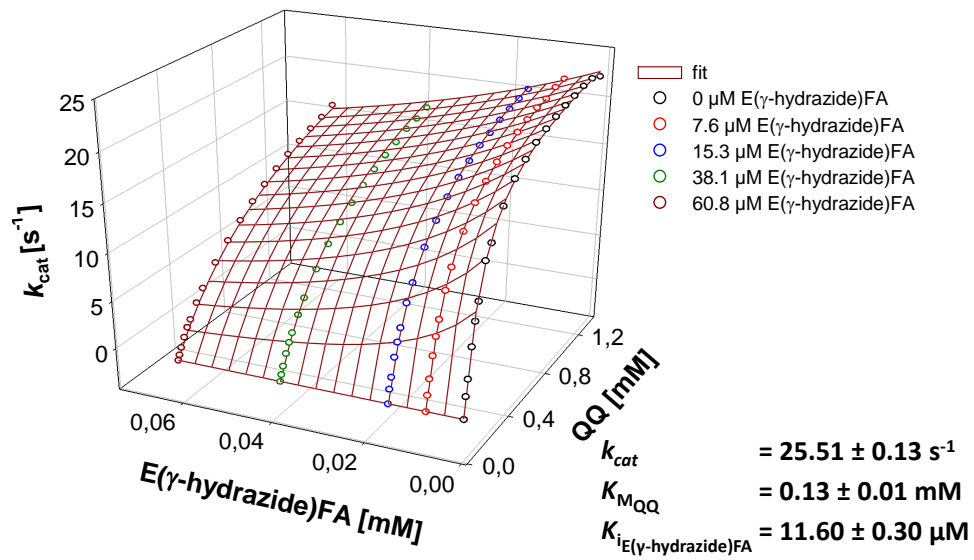

Figure 8.13: Three-dimensional analysis of E( $\gamma$-hydrazide)FA binding to hQC. Obtained data points from the continuous calorimetric activity assay were fitted according to Eq. 15 . 
Table 8.1: Determined bond length of the metal complex in hQC after coordination of different ligands.

\begin{tabular}{|c|c|c|c|c|c|}
\hline Bond length & $\begin{array}{l}\text { His330/NE2- } \\
\text { Zn(II) }\end{array}$ & $\begin{array}{l}\text { Glu202/OE2- } \\
\text { Zn(II) }\end{array}$ & $\begin{array}{l}\text { Asp159/OD2- } \\
\text { Zn(II) }\end{array}$ & $\begin{array}{l}\text { Ligand- } \\
\text { Zn(II) }\end{array}$ & $\begin{array}{l}\text { Ligand- } \\
\text { Zn(II) }\end{array}$ \\
\hline $\begin{array}{l}\mathrm{Zn}(\mathrm{II})-\mathrm{hQC} \\
\text { resting state }\end{array}$ & $2.12 \AA$ & $1.88 \AA$ & $2.05 \AA$ & $2.21 \AA$ & \\
\hline $\begin{array}{c}\mathrm{Zn}(\mathrm{II})-\mathrm{hQC} \\
\text { QFRH }\end{array}$ & $2.06 \AA$ & $1.70 \AA$ & $2.06 \AA$ & $2.10 \AA$ & \\
\hline $\begin{array}{l}\mathrm{Zn}(\mathrm{II})-\mathrm{hQC} \\
\text { pEFRH }\end{array}$ & $1.94 \AA$ & $1.96 \AA$ & $1.98 \AA$ & $1.85 \AA$ & \\
\hline $\begin{array}{l}\mathrm{Zn}(\mathrm{II})-\mathrm{hQC} \\
\text { EFRH }\end{array}$ & $2.11 \AA$ & $1.85 \AA$ & $2.02 \AA$ & $1.89 \AA$ & \\
\hline $\begin{array}{c}\text { isoDromeQC } \\
\text { EFRH (mono) }\end{array}$ & $1.96 \AA$ & $2.02 \AA$ & $2.02 \AA$ & $1.87 \AA$ & \\
\hline $\begin{array}{l}\text { isoDromeQC } \\
\text { EFRH (bi) }\end{array}$ & $1.96 \AA$ & $2.02 \AA$ & $2.02 \AA$ & $1.99 \AA$ & $2.17 \AA$ \\
\hline $\begin{array}{c}\mathrm{Zn}(\mathrm{II})-\mathrm{hQC} \\
\mathrm{E}(\gamma \text {-hydrazide }) \mathrm{FA}\end{array}$ & $2.09 \AA$ & $2.01 \AA$ & $2.10 \AA$ & $2.19 \AA$ & $2.34 \AA$ \\
\hline
\end{tabular}

Table 8.2: Determined angles of the metal complex in hQC after coordination of different ligands.

\begin{tabular}{|c|c|c|c|c|c|}
\hline Angles & $\begin{array}{l}\text { His330/NE2- } \\
\text { Zn(II)- } \\
\text { Glu202/OE2 }\end{array}$ & $\begin{array}{l}\text { Glu202/OE2- } \\
\text { Zn(II)- } \\
\text { Asp159/OD2 }\end{array}$ & $\begin{array}{c}\text { Asp159/OD2 } \\
\text { Zn(II)- } \\
\text { His330/NE2 }\end{array}$ & $\begin{array}{c}\text { Ligand- } \\
\text { Zn(II)- } \\
\text { His330/NE2 }\end{array}$ & $\begin{array}{c}\text { Ligand- } \\
\text { Zn(II)- } \\
\text { Asp159/OD2 }\end{array}$ \\
\hline $\begin{array}{l}\mathrm{Zn}(\mathrm{II})-\mathrm{hQC} \\
\text { resting state }\end{array}$ & $104.5^{\circ}$ & $104.2^{\circ}$ & $101.2^{\circ}$ & & \\
\hline $\begin{array}{c}\mathrm{Zn}(\mathrm{II})-\mathrm{hQC} \\
\text { QFRH }\end{array}$ & $118.4^{\circ}$ & $108.7^{\circ}$ & $102.5^{\circ}$ & & \\
\hline $\begin{array}{c}\mathrm{Zn}(\mathrm{II})-\mathrm{hQC} \\
\text { pEFRH }\end{array}$ & $118.4^{\circ}$ & $105.9^{\circ}$ & $98.9^{\circ}$ & & \\
\hline $\begin{array}{c}\mathrm{Zn}(\mathrm{II})-\mathrm{hQC} \\
\mathrm{EFRH}\end{array}$ & $112.0^{\circ}$ & $105.6^{\circ}$ & $102.3^{\circ}$ & $121.1^{\circ}$ & $120.1^{\circ}$ \\
\hline $\begin{array}{c}\text { isoDromeQC } \\
\text { EFRH } \\
\text { (mono) }\end{array}$ & $118.2^{\circ}$ & $105.3^{\circ}$ & $99.5^{\circ}$ & $121.1^{\circ}$ & $121.3^{\circ}$ \\
\hline
\end{tabular}


A
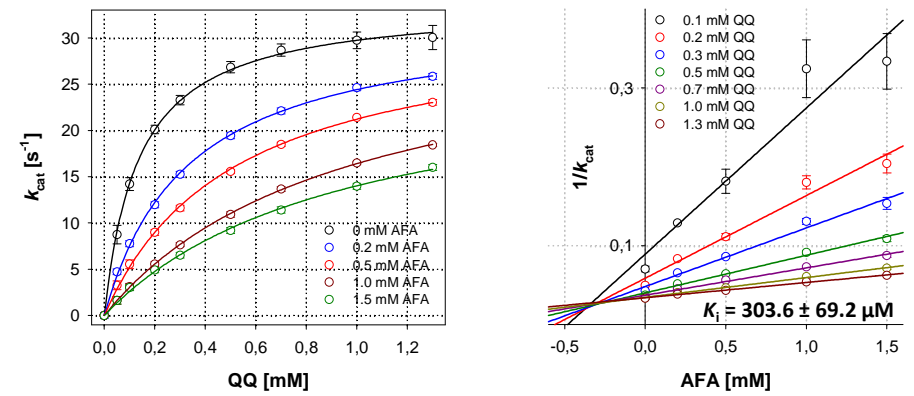

B


C
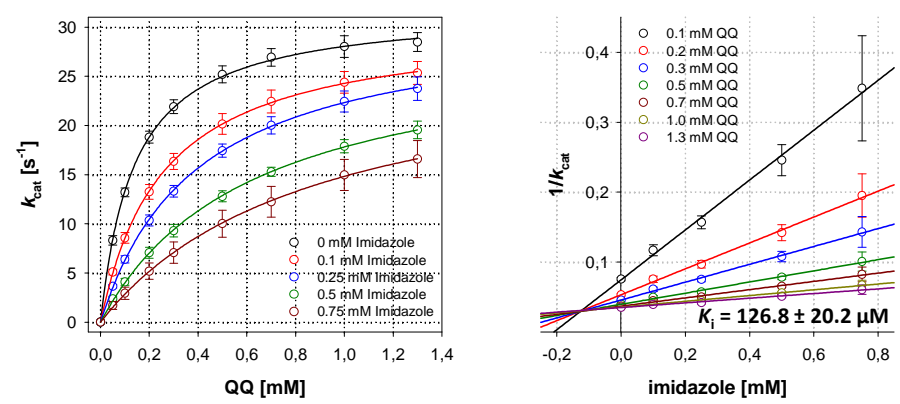

D
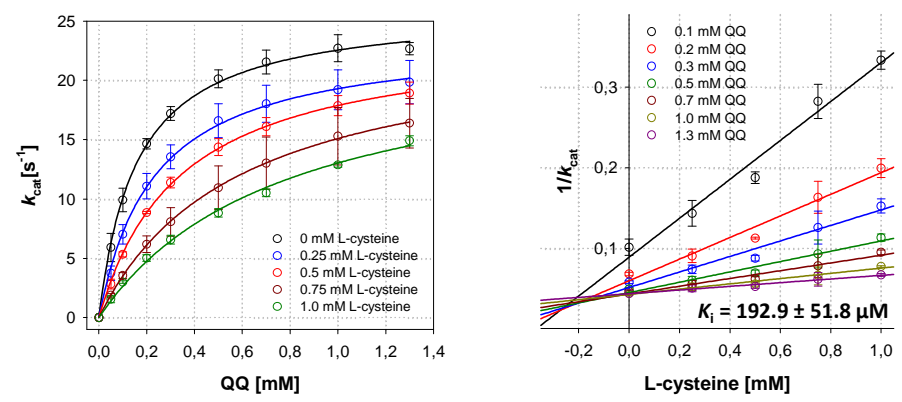

$\mathbf{E}$
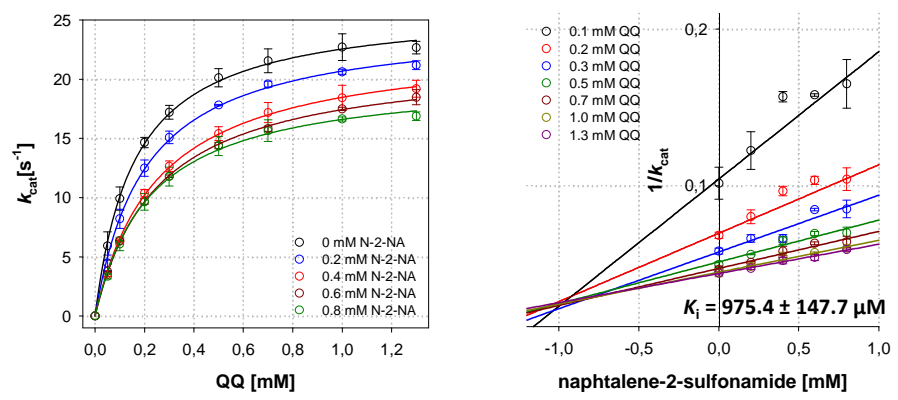

Figure 8.14: Kinetic characterization of hQC in presence of different inhibitors using the calorimetric activity assay. Michaelis-Menten plots of conversion of the N-terminal glutaminyl substrate (QQ) catalyzed by hQC utilizing different inhibitors. Obtained data points were fitted according to Eq. 3. In addition a Dixon plot for each inhibitor was performed. Obtained data points were fitted to a linear dependency and the $K_{\mathrm{i}}$ value was determined by calculating the intersections for each linear regression. Kinetic characterization of (A) the tripeptide AFA, (B) the product pEFRH (C) imidazole, (D) the amino acid L-cysteine and (E) the compound naphtalene-2-sulfonamide. 
Table 8.3: Crystallographic data and refinement statistics for hQC in resting state and in complex with different ligands.

hQC resting state

Data Collection

synchrotron
beamline

space group

cell dimensions

$$
\begin{aligned}
& \text { a, b, c }(\AA) \\
& \alpha, \beta, \gamma(\operatorname{deg})
\end{aligned}
$$

resolution $(\AA)$

$\mathrm{R}_{\text {sym }}, \mathrm{R}_{\text {merge }}$

$(\mathrm{I} / \sigma(\mathrm{I})$

completeness (\%)

redundancy

$\mathrm{CC}(1 / 2)$

Refinement

resolution $(\AA)$

no. of reflections

$\mathrm{R}_{\text {work }}, \mathrm{R}_{\text {free }}(\%)$

no. of atoms

protein (chain A/B)

$\mathrm{Zn}$

other ligands

water

B factor $\left(\AA^{2}\right)$

protein (chain A/B)

$\mathrm{Zn}$

other ligands

water

rmsd

bond length $(\AA)$

bond angles (deg)

B factor from the Wilson Plot $\left(\AA^{2}\right)$

\section{ESRF}

ID23-1

H32

119.1, 119.1, 333.6

90, 90, 120

100-2.14 (2.25-2.14)

9.6, 51.8

5.74

100.0

10.0

95.7

43.85-2.14

50620

$15.71,19.70$

$5791 / 5791$

2

53

442

$22.32 / 31.69$

20.36

55.16

32.78

0.009

1.168

32.27 
$\mathrm{hQC}+\mathrm{QFRH}$

Data Collection

synchrotron

MaxIV

beamline

1911-1

space group

H32

cell dimensions

$$
\begin{aligned}
& \mathrm{a}, \mathrm{b}, \mathrm{c}(\AA) \\
& \alpha, \beta, \gamma(\mathrm{deg})
\end{aligned}
$$

119.1, 119.1, 333.6

90, 90, 120

resolution $(\AA)$

100-1.90 (2.00-1.90)

$\mathrm{R}_{\text {sym }}, \mathrm{R}_{\text {merge }}$

$5.9,58.1$

$(\mathrm{I} / \sigma(\mathrm{I})$

2.45

completeness (\%)

99.7

redundancy

6.6

$\mathrm{CC}(1 / 2)$

77.9

Refinement

resolution $(\AA)$

55.69-1.90

no. of reflections

70280

$\mathrm{R}_{\text {work }}, \mathrm{R}_{\text {free }}(\%)$

$17.66,21.52$

no. of atoms

protein (chain A/B)

$5791 / 5791$

ligand $(\mathrm{Q})$ (chain A)

10

$\mathrm{Zn}$

2

other ligands

80

water

360

B factor $\left(\AA^{2}\right)$

protein (chain A/B)

$29.14 / 35.85$

ligand $(\mathrm{Q})$ (chain $\mathrm{A}$ )

41.81

$\mathrm{Zn}$

24.74

other ligands

42.32

water

35.45

rmsd

bond length $(\AA)$

0.008

bond angles (deg)

1.134

B factor from the Wilson Plot $\left(\AA^{2}\right)$

29.12 
$\mathrm{hQC}+\mathrm{pEFRH}$

Data Collection

synchrotron

MaxIV

beamline

1911-1

space group

H32

cell dimensions
a, b, c $(\AA)$
119.1, 119.1, 333.6
$\alpha, \beta, \gamma(\mathrm{deg})$
90, 90, 120

resolution $(\AA)$

100-1.88 (1.98-1.88)

$\mathrm{R}_{\text {sym }}, \mathrm{R}_{\text {merge }}$

$4.6,48.9$

$(\mathrm{I} / \sigma(\mathrm{I})$

2.46

completeness (\%)

99.2

redundancy

5.6

$\mathrm{CC}(1 / 2)$

77.2

Refinement

resolution $(\AA)$

$35.33-1.88$

no. of reflections

73902

$\mathrm{R}_{\text {work }}, \mathrm{R}_{\text {free }}(\%)$

$15.36,17.85$

no. of atoms

protein (chain A/B)

$5791 / 5791$

ligand $(\mathrm{pEF})$ (chain $\mathrm{A})$

19

$\mathrm{Zn}$

2

other ligands

113

water

351

B factor $\left(\AA^{2}\right)$

protein (chain A/B)

$31.83 / 40.41$

ligand (pEF) (chain A)

56.60

$\mathrm{Zn}$

22.62

other ligands

62.49

water

40.18

rmsd

bond length $(\AA)$

0.011

bond angles (deg)

1.329

B factor from the Wilson Plot $\left(\AA^{2}\right)$

26.61 
$\mathrm{hQC}+\mathrm{EFRH}$

\begin{tabular}{|c|c|}
\hline \multicolumn{2}{|l|}{ Data Collection } \\
\hline synchrotron & MaxIV \\
\hline beamline & $1911-1$ \\
\hline space group & H32 \\
\hline \multicolumn{2}{|l|}{ cell dimensions } \\
\hline $\mathrm{a}, \mathrm{b}, \mathrm{c}(\AA)$ & $119.1,119.1,333.6$ \\
\hline$\alpha, \beta, \gamma(\operatorname{deg})$ & $90,90,120$ \\
\hline resolution $(\AA)$ & $100-1.92(2.02-1.92)$ \\
\hline $\mathrm{R}_{\text {sym }}, \mathrm{R}_{\text {merge }}$ & $6.0,40.6$ \\
\hline$(\mathrm{I} / \sigma(\mathrm{I})$ & 2.47 \\
\hline completeness (\%) & 99.5 \\
\hline redundancy & 7.4 \\
\hline $\mathrm{CC}(1 / 2)$ & 77.6 \\
\hline \multicolumn{2}{|l|}{ Refinement } \\
\hline resolution $(\AA)$ & $43.92-1.92$ \\
\hline no. of reflections & 26583 \\
\hline $\mathrm{R}_{\text {work }}, \mathrm{R}_{\text {free }}(\%)$ & $17.92,20.88$ \\
\hline \multicolumn{2}{|l|}{ no. of atoms } \\
\hline protein (chain A/B) & $5791 / 5791$ \\
\hline ligand $(\mathrm{E})$ (chain A) & 10 \\
\hline $\mathrm{Zn}$ & 2 \\
\hline other ligands & 72 \\
\hline water & 439 \\
\hline \multicolumn{2}{|l|}{ B factor $\left(\AA^{2}\right)$} \\
\hline protein (chain A/B) & $32.68 / 40.14$ \\
\hline ligand $(\mathrm{E})$ (chain A) & 24.35 \\
\hline $\mathrm{Zn}$ & 19.55 \\
\hline other ligands & 34.94 \\
\hline water & 43.23 \\
\hline \multicolumn{2}{|l|}{ rmsd } \\
\hline bond length $(\AA)$ & 0.008 \\
\hline bond angles (deg) & 1.143 \\
\hline B factor from the Wilson Plot $\left(\AA^{2}\right)$ & 24.76 \\
\hline
\end{tabular}


hQC $+\mathrm{E}(\gamma$-hydrazide $) \mathrm{FA}$

Data Collection

synchrotron

DESY

beamline

P 13

space group

H32

cell dimensions

$$
\begin{aligned}
& \mathrm{a}, \mathrm{b}, \mathrm{c}(\AA) \\
& \alpha, \beta, \gamma(\mathrm{deg})
\end{aligned}
$$

$119.1,119.1,333.3$

$90,90,120$

resolution $(\AA)$

100-1.95 (2.05-1.95)

$\mathrm{R}_{\text {sym }}, \mathrm{R}_{\text {merge }}$

12.6, 74.8

$(\mathrm{I} / \sigma(\mathrm{I})$

2.45

completeness (\%)

100.0

redundancy

13.2

$\mathrm{CC}(1 / 2)$

82.6

Refinement

resolution $(\AA)$

64.92-1.95

no. of reflections

94868

$\mathrm{R}_{\text {work }}, \mathrm{R}_{\text {free }}(\%)$

$17.19,20.22$

no. of atoms

protein (chain A/B)

$5791 / 5791$

ligand ( $\mathrm{E}(\gamma$-hydrazide)FA) (chain A/B)

$27 / 27$

Zn

9

other ligands

108

water

383

B factor $\left(\AA^{2}\right)$

protein (chain A/B)

$25.71 / 29.45$

ligand (E( $\gamma$-hydrazide)FA) (chain A/B)

$21.62 / 27.18$

Zn

33.40

other ligands

42.56

water

34.66

rmsd

bond length $(\AA)$

0.008

bond angles (deg)

1.133

B factor from the Wilson Plot $\left(\AA^{2}\right)$

26.75 


\section{Literature}

\section{References}

[1] L. Haitinger. "Vorlige Mittheilung ber Glutaminsaure und Pyrrol". Monatshefte fr Chemie 3 (1882), pp. 228-229.

[2] A. Kumar and A. K. Bachhawat. "Pyroglutamic acid: Throwing light on a lightly studied metabolite". Current Science 102 (2012), pp. 288-297.

[3] S. Schilling et al. "Glutaminyl cyclases unfold glutamyl cyclase activity under mild acid conditions." Federation of European Biochemical Societies 563 (2004), pp. 191196.

[4] J. H. Perlman et al. "Hydrogen Bonding Interaction of Thyrotropin-releasing Hormone (TRH) with Transmembrane Tyrosine 106 of the TRH Receptor". The Journal of Biological Chemistry 269 (1994), pp. 1610-1613.

[5] F. Seifert et al. "Glutaminyl cyclases display significant catalytic proficiency for glutamyl substrates." Biochemistry 48 (2009), pp. 11831-11833.

[6] M. Messer. "Enzymatic Cyclization of L-Glutamine and L-Glutaminyl Peptides". Nature 4874 (1963), pp. 1299-1303.

[7] W. H. Busby et al. "An Enzyme(s) That Converts Glutaminyl-peptides into Pyroglutamylpeptides". The Journal of Biological Chemistry 262 (1987), pp. 8532-8536.

[8] W. H. Fischer and J. Spiess. "Identification of a mammalian glutaminyl cyclase converting glutaminyl into pyroglutamyl peptides." Proceedings of the National Academy of Sciences 84 (1987), pp. 3628-3632.

[9] I. Song. "Molecular cloning, sequence analysis and expression of human pituitary glutaminyl cyclase". Journal of Molecular Endocrinology 13 (1994), pp. 77-86.

[10] T. Pohl et al. "Primary structure and functional expression of a glutaminyl cyclase." Proceedings of the National Academy of Sciences 88 (1991), pp. 10059-10063.

[11] R. C. Bateman et al. "Evidence for essential histidines in human pituitary glutaminyl cyclase". Biochemistry 40 (2001), pp. 11246-11250. 
[12] B. Bennett and R. C. Holz. "EPR studies on the mono- and dicobalt(II)-substituted forms of the aminopeptidase from Aeromonas proteolytica. Insight into the catalytic mechanism of dinuclear hydrolases". Journal of the American Chemical Society 119 (1997), pp. 1923-1933.

[13] J. M. Prescott and H. Wilkes. "Aeromonas Aminopeptidase: Purification and Some General Properties". Archives of Biochemistry and Biophysics 117 (1966), pp. 328336.

[14] J. M. Prescott et al. "Spectral and kinetic studies of metal-substituted Aeromonas aminopeptidase: nonidentical, interacting metal-binding sites". Biochemistry 24 (1985), pp. 5350-5356.

[15] S. Schilling. "Charakterisierung der humanen Glutaminyl-Cyclase im Vergleich mit dem analogen Enzym aus Carica papaya". PhD Thesis (2004), pp. 1-42.

[16] B. Chevrier et al. "Crystal structure of Aeromonas proteolytica aminopeptidase: a prototypical member of the co-catalytic zinc enzyme family". Structure 2 (1994), pp. 283-291.

[17] Y. J. Dong et al. "Crystal structure of methyl parathion hydrolase from Pseudomonas sp. WBC-3". Journal of Molecular Biology 353 (2005), pp. 655-663.

[18] T. P. De Beer et al. "PDBsum additions". Nucleic Acids Research 42 (2014), pp. 292-296.

[19] K. F. Huang and Y. L. Liu. "Cloning, expression, characterization, and crystallization of a glutaminyl cyclase from human bone marrow: A single zinc metalloenzyme". Protein Expression and Purification 43 (2005), pp. 65-72.

[20] K. F. Huang et al. "Crystal structures of human glutaminyl cyclase, an enzyme responsible for protein N-terminal pyroglutamate formation." Proceedings of the National Academy of Sciences 102 (2005), pp. 13117-13122.

[21] S. Schilling et al. "Heterologous expression and characterization of human glutaminyl cyclase: evidence for a disulfide bond with importance for catalytic activity." Biochemistry 41 (2002), pp. 10849-10857. 
[22] D. Ruiz-Carrillo et al. "Structures of Glycosylated Mammalian Glutaminyl Cyclases Reveal Conformational Variability near the Active Center". Biochemistry 18 (2011), pp. $6280-6288$.

[23] S. Schilling et al. "Identification of human glutaminyl cyclase as a metalloenzyme. Potent inhibition by imidazole derivatives and heterocyclic chelators." The Journal of Biological Chemistry 278 (2003), pp. 49773-49779.

[24] G. N. Abraham and D. N. Podell. "Pyroglutamic acid. Non-metabolic formation, function in proteins and peptides, and characteristics of the enzymes effecting its removal". Molecular and Cellular Biochemistry 38 (1981), 181190.

[25] A. C. Awade et al. "Pyrrolidone Carboxyl Peptidase (Pcp): An Enzyme That Removes Pyroglutamic Acid (pGlu) From pGlu-Peptides and pGlu-Proteins". PROTEINS: Structure, Function, and Genetics 51 (1994), pp. 34-51.

[26] J. Boler et al. "The identity of chemical and hormonal properties of the thyrotropin releasing hormone and pyroglutamyl-histidyl-proline amide". Biochemical and Biophysical Research Communications 37 (1969), pp. 705-710.

[27] D. Lovejoy et al. "Distinct sequence of gonadotropin-releasing hormone (GnRH) in dogfish brain provides insight into GnRH evolution." Proceedings of the National Academy of Sciences 89 (1992), pp. 6373-6377.

[28] T. M. Bockers et al. "Glutaminyl-cyclase expression in the bovine/porcine hypothalamus and pituitary". Journal of Neuroendocrinology 7 (1995), pp. 445-453.

[29] E. Van Coillie et al. "Functional comparison of two human monocyte chemotactic protein-2 isoforms, role of the amino-terminal pyroglutamic acid and processing by CD26/dipeptidyl peptidase IV". Biochemistry 37 (1998), pp. 12672-12680.

[30] T. C. Saido et al. "Dominant and differential deposition of distinct $\beta$-amyloid peptide species, A $\beta \mathrm{N} 3(\mathrm{pE})$, in senile plaques". Neuron 14 (1995), pp. 457-466.

[31] A. Alzheimer. "er eine eigenartige Erkrankung der Hirnrinde". Allgemeine Zeitschrift fr Psychiatrie und Psychisch-gerichtliche Medizin 64 (1907), pp. 146-148.

[32] “Alzheimer' s Disease Facts and Figures". Alzheimer Association 10 (2014), pp. 175. 
[33] D. Selkoe. "The cell biology of $\beta$-amyloid precursor protein and presenilin in Alzheimer's disease". Trends in Cell Biology 8 (1998), pp. 447-453.

[34] J. Kang et al. "The precursor of Alzheimer's disease amyloid A4 protein resembles a cell-surface receptor". Nature 325 (1987), pp. 733-736.

[35] D. Selkoe et al. "Beta-amyloid precursor protein of Alzheimer disease occurs as 110to 135-kilodalton membrane-associated proteins in neural and nonneural tissues." Proceedings of the National Academy of Sciences 85 (1988), pp. 7341-7345.

[36] Y. Hung and D. J. Selkoe. "Selective ectodomain phosphorylation and regulated cleavage of beta-amyloid precursor protein." The EMBO journal 13 (1994), pp. 534542.

[37] J. Mills and P. B. Reiner. "Regulation of amyloid precursor protein cleavage". Journal of Neurochemistry 72 (1999), pp. 443-460.

[38] F. S. Esch. "Cleavage of amyloid beta peptide during constitutive processing of its precursor". Science 248 (1990), pp. 1122-1124.

[39] C. J Pike et al. "Amino-terminal Deletions Enhance Aggregation of Beta-Amyloid Peptides in Vitro". Biochemistry 270 (1995), pp. 23895-23898.

[40] E. Levy et al. "Mutation of the Alzheimer's disease Amyloid Gene in Heditary Cerebral Hemorrhage, Dutch Type". Science 248 (1990), pp. 1124-1126.

[41] D. Scheuner et al. "Secreted amyloid ß-protein similar to that in the senile plaques of Alzheimer's Disease is increased in vivo by the presenilin 1 and 2 and APP mutations linked to familial Alzheimer's Disease". Nature Medicine 2 (1996), pp. 864870.

[42] Y. M. Kuo et al. "Isolation, chemical characterization, and quantitation of A33pyroglutamyl peptide from neuritic plaques and vascular amyloid deposits." Biochemical and Biophysical Research Communications 237 (1997), pp. 188-191.

[43] R. Hosoda et al. "Quantification of Modified Amyloid beta Peptides in Alzheimer Disease and Down Syndrome Brains". Journal of Neuropathology and Experimental Neurology 57 (1998), pp. 1089-1095.

[44] C. Russo et al. "Heterogeneity of water-soluble amyloid B-peptide in Alzheimer's disease and Down's syndrome brains". FEBS Letters 409 (1997), pp. 411-416. 
[45] W. He and C. J. Barrow. "The Aß3-pyroglutamyl and 11-pyroglutamyl peptides found in senile plaque have greater beta-sheet forming and aggregation propensities in vitro than full-length Aß". Biochemistry 38 (1999), pp. 10871-10877.

[46] S. Schilling et al. "On the Seeding and Oligomerization of pGlu-Amyloid Peptides (in Vitro)". Biochemistry 45 (2006), pp. 12394-12399.

[47] D. Schlenzig et al. "Pyroglutamate formation influences solubility and amyloidogenicity of amyloid peptides." Biochemistry 48 (2009), pp. 7072-7078.

[48] Y. Harigaya et al. "Amyloid beta protein starting pyroglutamate at position 3 is a major component of the amyloid deposits in the Alzheimer's disease brain." Biochemical and Biophysical Research Communications 276 (2000), pp. 422-427.

[49] T.C. Saido. "Alzheimer's disease as proteolytic disorders: anabolism and catabolism of beta-amyloid". Neurobiology of Aging 19 (1998), pp. 69-75.

[50] C. Russo et al. "Pyroglutamate-modified amyloid ß-peptides - AßN3(pE) - strongly affect cultured neuron and astrocyte survival". Journal of Neurochemistry 82 (2002), pp. 1480-1489.

[51] T. Kuda et al. "Reduction of plasma glutamyl aminopeptidase activity in sporadic Alzheimer's disease". Biochemical and Biophysical Research Communications 231 (1997), pp. 526-530.

[52] N. Agirregoitia et al. "Effect of aging on rat tissue peptidase activities". Journal of Gerontology 58 (2003), pp. 792-797.

[53] M. Gololobov et al. "Steady-State Kinetics of Glutamine Cyclotransferase". Archives of Biochemistry and Biophysics 309 (1994), pp. 395-398.

[54] S. Schilling et al. "Substrate specificity of glutaminyl cyclases from plants and animals." Biological Chemistry 384 (2003), pp. 1583-1592.

[55] K. F. Huang et al. "A conserved hydrogen-bond network in the catalytic centre of animal glutaminyl cyclases is critical for catalysis." The Biochemical Journal 411 (2008), pp. 181-190.

[56] M. Buchholz et al. "The first potent inhibitors for human glutaminyl cyclase: synthesis and structure-activity relationship." Journal of Medicinal Chemistry 49 (2006), pp. 664-677. 
[57] K. S. Lundberg et al. "High-fidelity amplification using a thermostable DNA polymerase isolated from P.furiosus". Gene 108 (1991), pp. 1-6.

[58] C. Aaij and P. Borst. "The gel electrophoresis of DNA." Biochimica et Bophysica Acta 269 (1972), pp. 192-200.

[59] H. Inoue et al. "High efficiency transformation of Escherichia coli with plasmids". Gene 96 (1990), pp. 23-28.

[60] G. Bertani. "Studies on lysogenesis. I. The mode of phage liberation by lysogenic Escherichia coli." Journal of Bacteriology 62 (1951), pp. 293-300.

[61] D. Hanahan. "Studies on transformation of Escherichia coli with plasmids." Journal of Molecular Biology 166 (1983), pp. 557-580.

[62] F. W. Studier. "Protein production by auto-induction in high-density shaking cultures". Protein Expression and Purification 41 (2005), pp. 207-234.

[63] U. K. Laemmli. "Cleavage of structural proteins during the assembly of the head of bacteriophage T4." Nature 227 (1970), pp. 680-685.

[64] K. Weber and M. Osborn. "The Reliability of Molecular Weight Determinations by Dodecyl Sulfate-Polyacrylamide Gel Electrophoresis". The Journal of Biological Chemistry 244 (1969), pp. 4406-4412.

[65] S. Gill and P. Hippel. "Calculation of Extinction Coefficient from Amino Acid Sequence". Analytical Biochemistry 182 (1989), pp. 319-326.

[66] R. C. Bateman. "A spectrophotometric assay for glutaminyl-peptide cyclizing enzymes." Journal of Neuroscience Methods 30 (1989), pp. 23-28.

[67] G. D. Watt. "A microcalorimetric procedure for evaluating the kinetic parameters of enzyme-catalyzed reactions: Kinetic measurements of the nitrogenase system". Analytical Biochemistry 187 (1990), pp. 141-146.

[68] L. Sica et al. "A flow microcalorimetric method for enzyme activity measurements: application to dihydrofolate reductase." Analytical Biochemistry 165 (1987), pp. 341-348.

[69] K. F. Ellis and Morrison J. F. "Buffers of Constant Ionic Strength for Studying pH-Dependent Processes". Methods of Enzymology 45 (1982), pp. 405-426. 
[70] M. Dixon. "The determination of enzyme inhibitor constants." The Biochemical Journal 55 (1953), pp. 170-171.

[71] A. Schellenberger. "Enzymkatalyse - Einführung in die Chemie, Biochemie und Technologie der Enzyme." Springer Verlag 1 (1989), pp. 72-132.

[72] C. N. Pace et al. "Conformational stability and thermodynamics of folding of ribonucleases Sa, Sa2 and Sa3." Journal of Molecular Biology 279 (1998), pp. 271286.

[73] J. L. Cole and J. C. Hansen. "Analytical Ultracentrifugation as a Contemporary Biomolecular Research Tool”. Journal of Biomolecular Techniques 10 (1999), pp. $163-176$.

[74] A. Shevchenko et al. "Mass Spectrometric Sequencing of Proteins from SilverStained Polyacrylamide". Analytical Chemistry 68 (1996), pp. 850-858.

[75] A. Shevchenko et al. "In-gel digestion for mass spectrometric characterization of proteins and proteomes." Nature protocols 1 (2006), pp. 2856-2860.

[76] W. Kabsch. "Integration, scaling, space-group assignment and post-refinement". Biological Crystallography 66 (2010), pp. 133-144.

[77] W. Kabsch. "XDS". Biological Crystallography 66 (2010), pp. 125-132.

[78] A. J. McCoy et al. "Phaser crystallographic software". Journal of Applied Crystallography 40 (2007), pp. 658-674.

[79] P. D. Adams et al. "PHENIX: A comprehensive Python-based system for macromolecular structure solution". Biological Crystallography 66 (2010), pp. 213-221.

[80] P. Emsley et al. "Features and development of Coot." Biological crystallography 66 (2010), pp. 486-501.

[81] E. F. Pettersen et al. "UCSF Chimera - A visualization system for exploratory research and analysis". Journal of Computational Chemistry 25 (2004), pp. 16051612.

[82] A. W. Schüttelkopf and D. M. Van Aalten. "PRODRG: A tool for high-throughput crystallography of protein-ligand complexes". Biological Crystallography 60 (2004), pp. $1355-1363$. 
[83] F. Agostini et al. "ccSOL omics: a webserver for large-scale prediction of endogenous and heterologous solubility in E. coli". Bioinformatics 30 (2014), pp. 2975-2977.

[84] W. G. Kaelin et al. "Expression cloning of a cDNA encoding a retinoblastoma binding protein with E2F-like properties". Cell 70 (1992), pp. 351-364.

[85] R. E. Booth et al. "Human pituitary glutaminyl cyclase: expression in insect cells and dye affinity purification". Protein Expression and Purification 32 (2003), pp. 141146.

[86] J.B. Perrin. "Brownian movement of an ellipsoid. I. Dielectric dispersion of an ellipsoidal molecule". Journal de Physique et le Radium 6 (1934), pp. 497-511.

[87] S. Schilling et al. "Continuous spectrometric assays for glutaminyl cyclase activity." Analytical Biochemistry 303 (2002), pp. 49-56.

[88] M. J. Todd and J. Gomez. "Enzyme kinetics determined using calorimetry: a general assay for enzyme activity?" Analytical Biochemistry 296 (2001), pp. 179-187.

[89] M. Eigen. "Proton Transfer, Acid-Base Catalysis, and Enzymatic Hydrolysis." Angewandte Chemie 3 (1964), pp. 1-72.

[90] R. C. Weast. "Handbook of Chemistry and Physics ". CRC Press 1 (1964).

[91] S. E. Leeman. "The Isolation of a New Hypotensive from Bovine Hypothalami". Journal of Biological Chemistry 248 (1973), pp. 6854-6861.

[92] S. Y. Ying. "Inhibins, activins, and follistatins: gonadal proteins modulating the secretion of follicle-stimulating hormone." Endocrine Reviews 9 (1988), pp. 267293.

[93] M. Hungs and E. Mignot. "Hypocretin/orexin, sleep and narcolepsy". BioEssays 23 (2001), pp. 397-408.

[94] D. Schlenzig et al. "Pyroglutamate formation influences solubility and amyloidogenicity of amyloid peptides". Biochemistry 48 (2009), pp. 7072-7078.

[95] S. Jawhar et al. "Overexpression of glutaminyl cyclase, the enzyme responsible for pyroglutamate Aßformation, induces behavioral deficits, and glutaminyl cyclase knock-out rescues the behavioral phenotype in 5XFAD mice". Journal of Biological Chemistry 286 (2011), pp. 4454-4460. 
[96] M. L. Garcia-Martin et al. "Mapping Extracellular pH in Rat Brain Gliomas in Vivo by Magnetic Resonance Spectroscopic Imaging: Comparison with Maps of Metabolites". Cancer Research 61 (2001), pp. 6524-6531.

[97] H. Cynis et al. "Pyroglutamate-Amyloid- $\beta$ and Glutaminyl Cyclase Are Colocalized with Amyloid- $\beta$ in Secretory Vesicles and Undergo Activity-Dependent, Regulated Secretion." Neurodegenerative Diseases 14 (2014), pp. 85-97.

[98] B. L. Vallee et al. "The Release of Zinc from Carboxypeptidase and its Replacement". Journal of the Chemical Society 83 (1958), pp. 4-5.

[99] A. Piontek. "Deciphering the Catalytic Mechanism of the Zn-Enzyme Glutaminyl Cyclase and the Deduction of Transition-state analogue Inhibitors". PhD thesis. 2014, pp. 1-161.

[100] A. Stephan et al. "Mammalian glutaminyl cyclases and their isoenzymes have identical enzymatic characteristics." The FEBS Journal 276 (2009), pp. 6522-6536.

[101] S. Schilling et al. "Isolation, Catalytic Properties, and Competitive Inhibitors of the Zinc-Dependent Murine Glutaminyl Cyclase". Biochemistry 44 (2005), pp. 1341513424.

[102] Housecroft C. A. and Sharpe A. G. Pearson Studium (2006).

[103] J. H. Mills et al. "Computational Design of an Unnatural Amino Acid Dependent Metalloprotein with Atomic Level". Journal of the American Chemical Society 135 (2013), pp. 13393-13399.

[104] M. L. Zastrow and V. L. Pecoraro. "Designing hydrolytic zinc metalloenzymes." Biochemistry 53 (2014), pp. 957-978.

[105] David W. Christianson. "Structural Biology of Zinc". Vol. 42. Academic Press, 1991.

[106] H. Y. Cho et al. "Role of Divalent Metal Ions on Activity and Stability of Thermostable Dipeptidase from Bacillus stearothermophilus". Bioscience, Biotechnology and Biochemistry 61 (1997), pp. 1688-1692.

[107] K. A. Mccall et al. "Function and Mechanism of Zinc Metalloenzymes". The Journal of Nutrition (2000), pp. 1455-1458. 
[108] M. Laitaoja, J. Valjakka, and J. Jänis. "Zinc coordination spheres in protein structures". Inorganic Chemistry 52 (2013), pp. 10983-10991.

[109] Rardin R.L. and Lippard S.J. "Monodentate Carboxylate Complexes And The Carboxylate Shift - Implications For Polymetalloprotein Structure And Function". New Journal of Chemistry 15 (1991), pp. 417-430.

[110] A. C. Rosenzweig et al. "Geometry of the soluble methane monooxygenase catalytic diiron center in two oxidation states". Chemistry and Biology 2 (1995), pp. 409418.

[111] B. F. Gherman et al. "Dioxygen Activation in Methane Monooxygenase: A Theoretical Study". Journal of the American Chemical Society 126 (2004), pp. 29782990.

[112] A. Demsar et al. "Variable-temperature nuclear magnetic resonance spectroscopy allows direct observation of carboxylate shift in zinc carboxylate complexes". Journal of the American Chemical Society 124 (2002), pp. 3951-3958.

[113] C. A. Housecroft and A. G. Sharpe. "Inorganic Chemistry". Vol. 3. Pearson Studium, 2008, pp. 43-44.

[114] J. L. Kyung et al. "A New Type of Carboxypeptidase A Inhibitors Designed Using an Imidazole as a Zinc Coordinating Ligand". Bioorganic and Medicinal Chemistry 5 (1997), pp. 1989-1998.

[115] S. Schilling et al. "Glutaminyl cyclase inhibition attenuates pyroglutamate Abeta and Alzheimer's disease-like pathology." Nature Medicine 14 (2008), pp. 1106-1111.

[116] M. Buchholz et al. "Inhibitors for human glutaminyl cyclase by structure based design and bioisosteric replacement." Journal of Medicinal Chemistry 52 (2009), pp. 7069-7080.

[117] K. F. Huang et al. "Structures of human Golgi-resident glutaminyl cyclase and its complexes with inhibitors reveal a large loop movement upon inhibitor binding." The Journal of Biological Chemistry 286 (2011), pp. 12439-12449.

[118] J. Day and S. M. Cohen. "Investigating the selectivity of metalloenzyme inhibitors". Journal of Medicinal Chemistry 56 (2013), pp. 7997-8007. 
[119] S. Ahrland et al. "The relative affinities of ligand atoms for acceptors molecules and ions". Quarterly Review of the Chemical Society 12 (1958), pp. 265-276.

[120] N. Pace and E. Weerapana. "Zinc-Binding Cysteines: Diverse Functions and Structural Motifs". Biomolecules 4 (2014), pp. 419-434.

[121] N. Gavande et al. "Novel Cyclic Phosphinic Acids as GABAC $\rho$ Receptor Antagonists: Design, Synthesis, and Pharmacology." ACS Medicinal Chemistry Letters 2 (2011), pp. 11-16.

[122] R. J. Henry. "The mode of action of sulfonamides". Bacteriology Reviews 30 (1944), pp. $175-262$.

[123] S. Y. Low et al. "Effect of L-glutamate-gamma-hydrazide on the transport and metabolism of L-glutamine in rat liver cells and isolated mitochondria". Biochemical Society Transactions 18 (1990), pp. 1239-1240.

[124] S. Berry. "Correlation of rates of intramolecular tunneling processes, with application to some group V compounds". The Journal of Chemical Physics 32 (1960), pp. 933-938.

[125] A. W. Addison and T. N. Rao. "Synthesis, Structure, and Spectroscopic Properties of Copper(II) Compounds containing Nitrogen-Sulphur Donor Ligands; the Crystal and Molecular Structure of Aqua[1,7-bis(N-methylbenzimidazol-2'-yl)-2,6dithiaheptane]copper(II) Perchlorate". Journal of the Chemical Society 251 (1984), pp. 1349-1356.

[126] K. N. Trueblood et al. "Atomic Dispacement Parameter Nomenclature. Report of a Subcommittee on Atomic Displacement Parameter Nomenclature". Acta Crystallographica 52 (1996), pp. 770-781.

[127] W. B. Turnbull and A. H. Daranas. "Can Low Affinity Systems Be Studied by Isothermal Titration Calorimetry?" Journal of the American Chemical Society 125 (2003), pp. 14859-14866.

[128] S. W. Dahl et al. "Carica papaya glutamine cyclotransferase belongs to a novel plant enzyme subfamily: cloning and characterization of the recombinant enzyme." Protein Expression and Purification 20 (2000), pp. 27-36. 
[129] D. R. Carrillo et al. "Kinetic and structural characterization of bacterial glutaminyl cyclases from Zymomonas mobilis and Myxococcus xanthus." Biological Chemistry 391 (2010), pp. 1419-1428.

[130] R. Wintjens et al. "Crystal structure of papaya glutaminyl cyclase, an archetype for plant and bacterial glutaminyl cyclases." Journal of Molecular Biology 357 (2006), pp. $457-470$.

[131] S. Zerhouni et al. "Purification and characterization of papaya glutamine cyclotransferase , a plant enzyme highly resistant to chemical , acid and thermal denaturation". Biochemica et Biophysica Acta 1387 (1998), pp. 275-290.

[132] T. Guevara et al. "Papaya glutamine cyclotransferase shows a singular five-fold $\beta$ propeller architecture that suggests a novel reaction mechanism". Biological Chemistry 387 (2006), pp. 1479-1486.

[133] B. Koch et al. "Crystal structures of glutaminyl cyclases (QCs) from Drosophila melanogaster reveal active site conservation between insect and mammalian QCs." Biochemistry 51 (2012), pp. 7383-7392.

[134] A. El Moussaoui et al. "Revisiting the enzymes stored in the laticifers of Carica papaya in the context of their possible participation in the plant defence mechanism." Cellular and Molecular Life Sciences 58 (2001), pp. 556-570.

[135] S. Schilling et al. "Isolation and characterization of glutaminyl cyclases from Drosophila: evidence for enzyme forms with different subcellular localization." Biochemistry 46 (2007), pp. 10921-10930.

[136] S. F. Sousa et al. "The carboxylate shift in zinc enzymes: A computational study". Journal of the American Chemical Society 129 (2007), pp. 1378-1385.

[137] S. F. Sousa et al. "Enzymatic "tricks": Carboxylate shift and sulfur shift". International Journal of Quantum Chemistry 114 (2014), pp. 1253-1256.

[138] S. F. Sousa et al. "Farnesyltransferase-new insights into the zinc-coordination sphere paradigm: evidence for a carboxylate-shift mechanism." Biophysical Journal 88.1 (2005), pp. 483-494. 
[139] M. Högbom et al. "Crystal structures of oxidized dinuclear manganese centres in Mn-substituted class I ribonucleotide reductase from Escherichia coli: Carboxylate shifts with implications for O2 activation and radical generation". Journal of Biological Inorganic Chemistry 6 (2001), pp. 315-323.

[140] R. H. Holm et al. "Structural and Functional Aspects of Metal Sites in Biology". Chemical Reviews 96 (1996), pp. 2239-2314.

[141] F. Bard et al. "Peripherally administered antibodies against amyloid beta-peptide enter the central nervous system and reduce pathology in a mouse model of Alzheimer disease." Nature Medicine 6 (2000), pp. 916-919.

[142] R. B. DeMattos et al. "Peripheral anti-A beta antibody alters CNS and plasma A beta clearance and decreases brain A beta burden in a mouse model of Alzheimer's disease." Proceedings of the National Academy of Sciences 98 (2001), pp. 88508855. 


\section{Acknowledgement}

First of all, I would like to thank Prof. Dr. Kai Tittmann who has given me the opportunity to work in the Department of Molecular Enzymology, especially on this very interesting topic. During my doctoral thesis he was a great supervisor and I want to thank him for contribution of numerous ideas and constructive discussions. Furthermore, I would like to thank him for getting a better understanding of biochemical processes in general and to teach me fast kinetic methods.

Second, I would like to thank Prof. Dr. Franc Meyer for taking part in the thesis committee and for his external co-supervision during this doctoral thesis. In addition, I liked the critical discussions and support concerning to design high affinity inhibitors for metalloenzymes.

Thirdly, I would like to thank Prof. Dr. Derek Logan for his external co-supervision during this doctoral thesis and for taking part in the thesis committee. Also, I would like to thank him to gave me the possibility to work in his department and for usage of his instruments. I really enjoyed the trip to Lund.

Furthermore, I am grateful to Jun. Prof. Dr. Ricardo Mata, Prof. Dr. Claudia Höbartner, Dr. Franziska Thomas and Dr. Fabian Commichau for being members of my extended Thesis Committee.

I would also like to thank Prof. Dr. Ralf Ficner for the generous ability to use the instruments and facilities of his department.

I am very grateful to Prof. Dr. Ulf Diederichsen for a nice and uncomplicated collaboration in the development and synthesis of potential human glutaminyl cyclase inhibitors. Furthermore, he gave me the opportunity to use the instruments and facilities of his department.

Many thanks to Brigitte Worbs for the synthesis of the compound 3-AMOHP 
I am thankful to Dr. Piotr Neumann for his support and teaching in theoretical and practical aspects of crystallography.

Many thanks to Prof. Dr. Ute Curth from the Hannover Medical School to give us the opportunity to perform analytical ultracentrifugation experiments in her department and helping me in data analysis.

I acknowledge Dr. Oliver Valerius for his assistance in the field of mass spectrometry. He made it possible to measure and to evaluate data whenever I needed and thus contributed to the flow of the work.

Dr. Florian Brodhun, Viktor Sautner and Sina Kristin Stumpf I acknowledge for proofreading of the thesis.

I would like to thank all former and present members of the Department of Molecular Enzymology for their help in the lab and the great working atmosphere.

Finally, I am deeply grateful to my parents for their endless support during my studies and life in general. 


\section{Curriculum vitae}

\section{Oliver Kupski}

\section{Personal Data:}

Address Arndtstraße 26,37075 Göttingen, Germany

Date of Birth $\quad 07 / 07 / 1984$

Nationality German

Marital status single

E-Mail_okupski@gwdg.de

\section{Education}

1991 - 1995 Herman-Nohl-Schule, Göttingen (primary school)

1995 - 1997 Bert-Brecht-Schule, Göttingen (orientation phase)

1997 - $2001 \quad$ Voigt-Realschule, Göttingen (secondary school)

2001 - 2004 Ludwig Witthuhn GmbH \& Co, Göttingen (education as a carpenter)

2004 - 2007 Abendgymnasium, Göttingen (secondary school; qualification: A-level)

\section{University Education}

2007 - 2010 Graduate in the degree program "Bachelor of Science in Biology" at the Georg-August-University Göttingen

Bachelor Thesis: "Expression, Purification, Crystallization and Interaction Analysis of Importin- $\beta$ in Complex with $\mathrm{IBB}_{S P N 1}$ or $\mathrm{IBB}_{\alpha} "$

2010 - 2012 Graduate in the degree program "Master of Science in Biochemistry and Microbiology" at the Georg-August-University Göttingen

Master Thesis: "Characterisation of the membrane binding mechanism of the membrane anchor (alpha peptide) of pyruvate oxidase from Escherichia coli" 
2013 - 2016 PhD student, Georg-August-University Göttingen, Department of Molecular Enzymology, PhD Thesis: „Structure, Mechanism and Inhibition of Human Glutaminyl Cyclase“

\section{Work Experience}

$2005-2011 \quad$ Technical lab assistant at MVZ wagnerstibbe für Laboratoriumsmedizin, Gynäkologie, Humangenetik und Pathologie GmbH

2011 - 2013 Technical lab assistant at Seqlab Sequence Laboratories Göttingen $\mathrm{GmbH}$

\section{Grant}

04/2013 - 04/2016 Member of the International PhD program "Metal Sites in Biomolecules: Structures, Regulation and Mechanisms"

\section{Research Stays}

$02-$ 03/2015 Four-week stay in the lab of Prof. Dr. Derek Logan (Lund University, Sweden)

\section{Scientific Skills}

Fundamentals of molecular biology (cloning, PCR, mutagenesis), heterologous expression and purification of proteins (FPLC, HPLC), enzyme kinetics (classic, stopped-flow, quenched flow), spectroscopic Methods (UV/VIS, CD, fluorescence, basics of NMR), SPR, MST, X-ray crystallography, ITC, MALS

\section{Teaching Experience}

Supervision of practical courses, lab rotations and a Master Thesis

\section{Additional Skills}

Computing Windows and Linux, MS Office, Origin, SigmaPlot, LaTex, crystallographic software (CCP4 suite, PHENIX suite, COOT, PYMOL, XDS), CHEMSK 
Languages German - native

English - advanced in written and spoken

French - beginner in written and spoken

March 22, 2017 
